\title{
NUMERICAL MODELING OF BRONZE COATING FORMATION USING CONCENTRATION DEPENDENT DIFFUSIVITY
}

By

\author{
Guilherme Garrido Damaceno
}

A thesis submitted to

The Faculty of Graduate Studies

In partial fulfillment of the requirement for the degree of MASTER OF SCIENCE

\author{
Department of Mechanical Engineering \\ The University of Manitoba \\ Winnipeg, Manitoba
}

October 2016

Copyright (C) 2016 by Guilherme Garrido Damaceno 


\begin{abstract}
The engineering of a component's surface is of remarkable importance, it allows applications that surpass what is achievable by the substrate alone by improving surface properties such as hardness, wear and corrosion resistance and even aesthetic value. This project focuses on obtaining bronze coatings through the diffusion of electrodeposited tin on copper via heat treatment. Specifically, the single phase $\alpha$ is targeted due to its attractive properties. Determining the correct heat treatment parameters is crucial since only a specific range of compositions lead to the formation of the desired microstructure and, consequently, properties. The diffusion of a thin coating of tin onto copper often leads to the formation of undesirable features: small craters containing different phases than the one targeted. In the present work, the diffusion behavior of tin-copper couple was investigated; a numerical model was developed to predict the composition profile after heat treatment, which agrees with experimental observations; and the mechanism for the formation of undesirable surface craters during heat treatment was studied and the probable solution proposed.
\end{abstract}




\section{Acknowledgements}

I would like to thank my advisor, Prof. Olanrewaju Ojo, for his guidance, knowledge, strong leadership and for accepting me as his student, making this great experience possible.

Also, big thanks to Mike Boskwick and Trevor Smith for their help in and around the lab, to my family for their paramount support, to my friends, both old and new, for their companionship and lastly but certainly not least, my wife Natália, for being a constant incentive for me to improve myself. 


\section{Table of Contents}

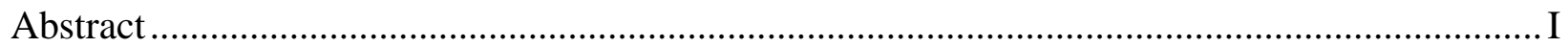

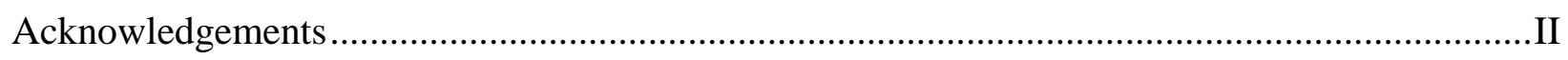

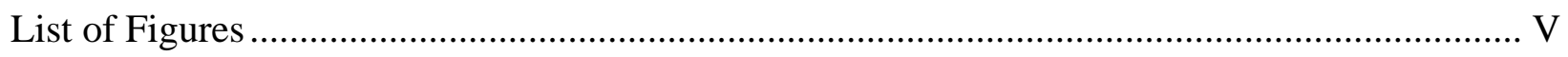

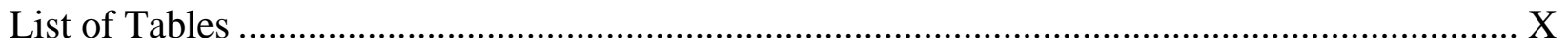

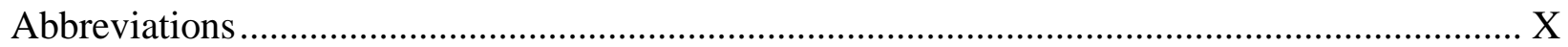

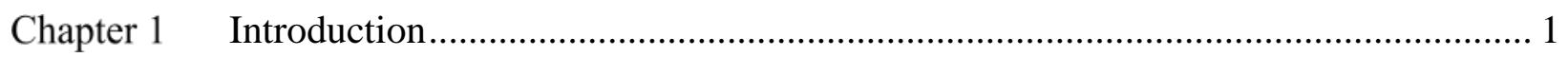

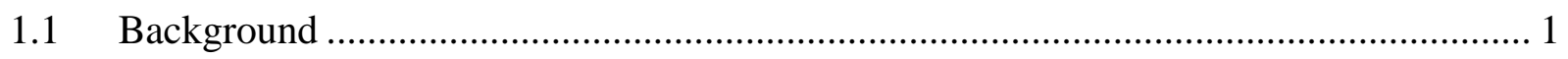

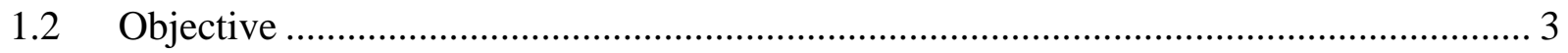

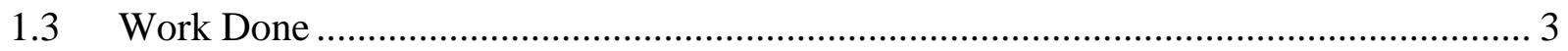

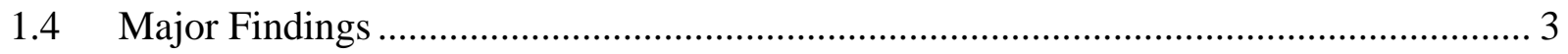

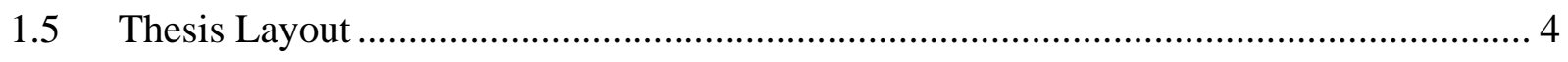

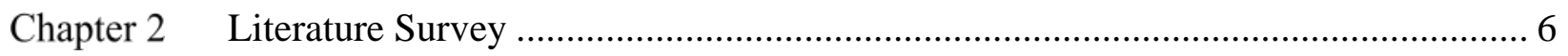

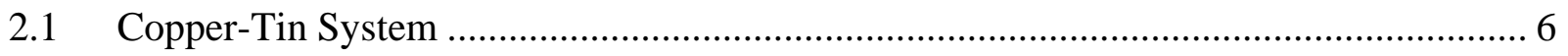

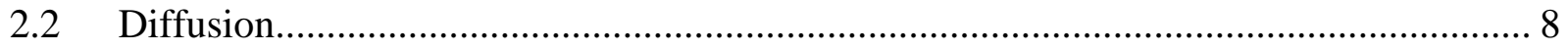

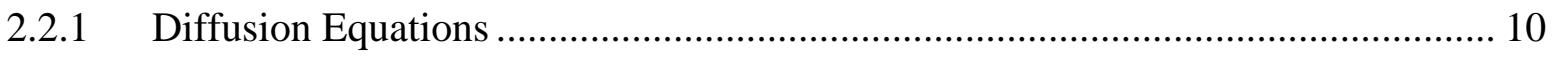

2.2.2 Diffusion Mechanisms ............................................................................. 12

2.2.3 Types of Diffusion Coefficients................................................................. 14

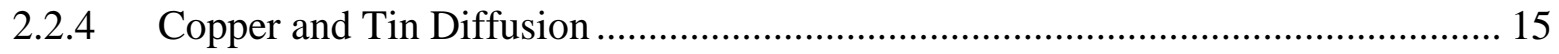

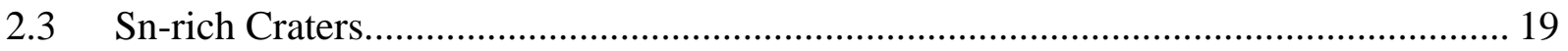




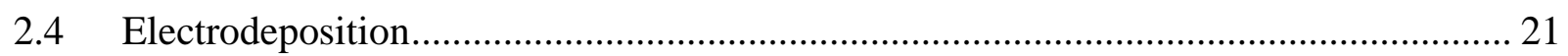

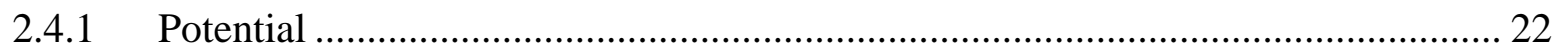

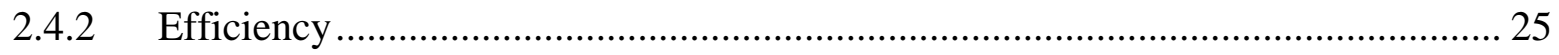

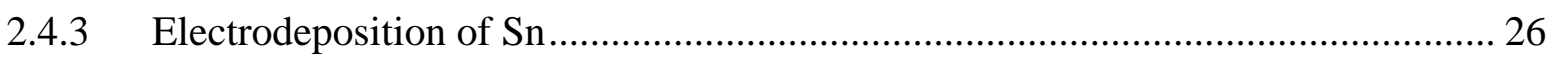

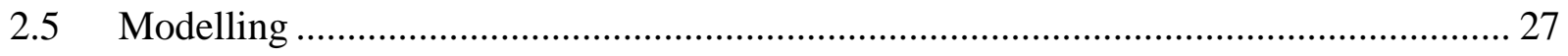

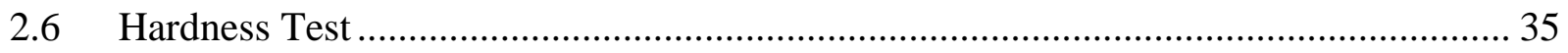

2.7 Objective of Current Research ………………......................................................... 36

Chapter 3 Numerical Modeling, Results and Discussion ......................................................... 37

3.1 High Temperature Heat Treatment Process Behavior of $\mathrm{Cu}-\mathrm{Sn}$..................................... 37

3.2 Development of Numerical Model............................................................................. 43

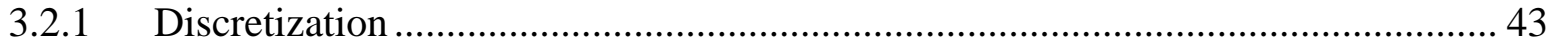

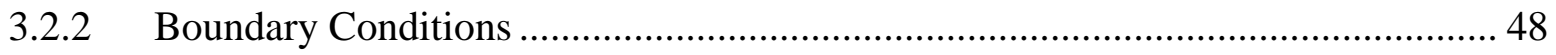

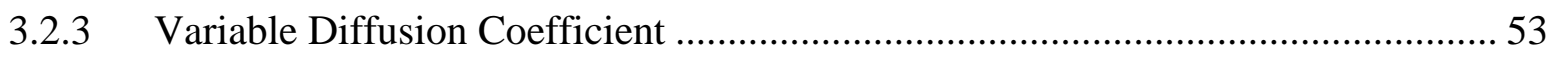

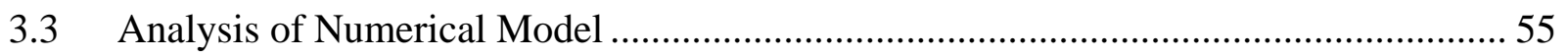

3.3.1 Comparison Against Finite/Semi-infinite Solid Diffusion ....................................... 55

3.3.2 Comparison Against Semi-infinite Composite Medium.......................................... 62

3.4 Modelling Heat Treatment Process Behavior …………….............................................. 62

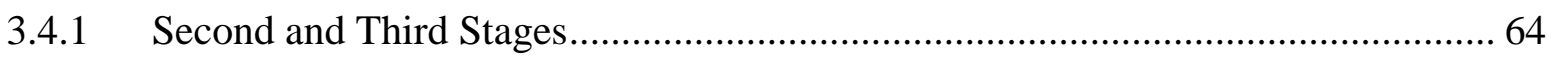

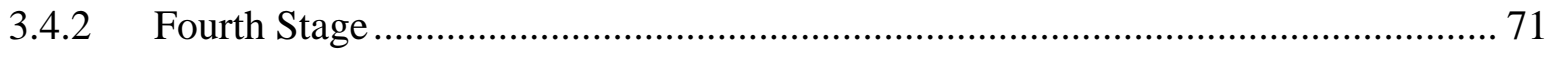

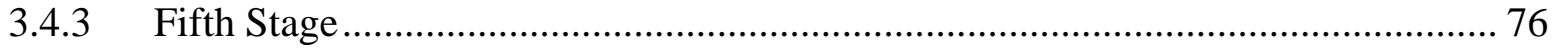




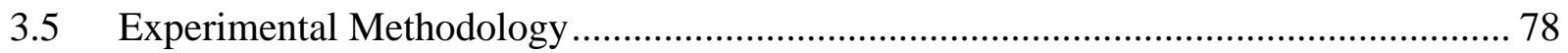

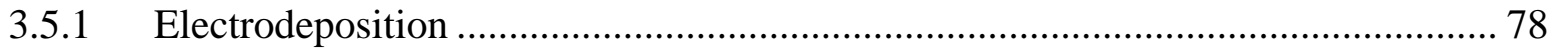

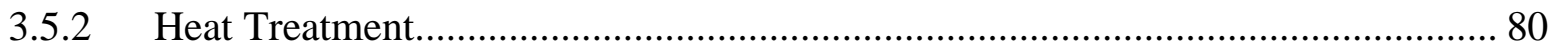

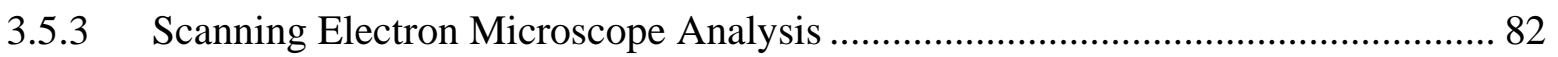

3.6 Experimental Results and Comparison with Simulation Results.................................... 82

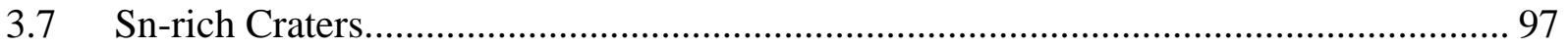

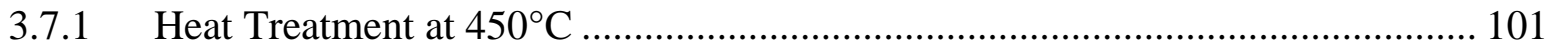

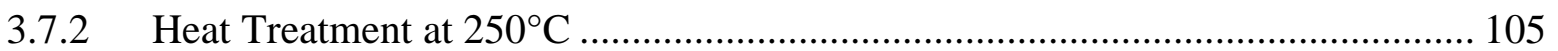

3.7.3 Proposed Mechanism of Crater Formation ...................................................... 108

3.8 Hardness Measurement ............................................................................................ 109

Chapter 4 Conclusions and Recommendations for Future Work ........................................... 111

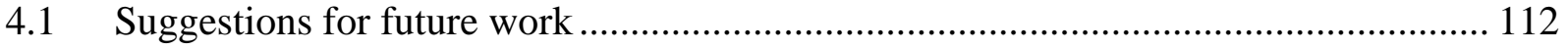

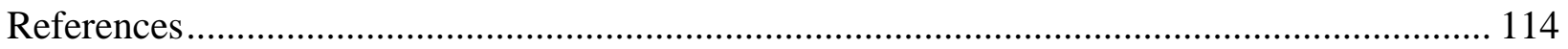

\section{List of Figures}

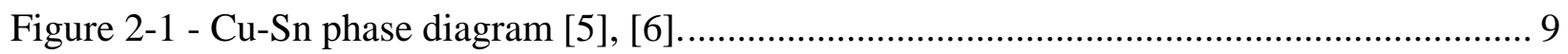

Figure 2-2 - Lattice sites (occupied by gray spheres) and interstitial sites (white space between spheres or location of black atom). Diffusion through interstitial and vacancy mechanisms are illustrated by the movement of atoms "a" and "b", respectively....................................................... 13 Figure 2-3 - Self-diffusion coefficients of solid $\mathrm{Cu}$ [25] and Sn (c and a-axis) [26] and liquid Sn [27]. 
Figure 2-4 - Interdiffusion activation energy (right axis) and frequency factor (left axis) as a

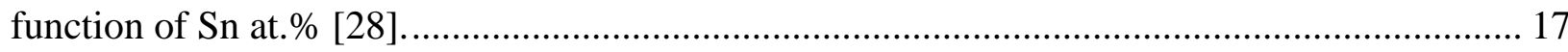

Figure 2-5 - Values for $\mathrm{Cu}$ and $\mathrm{Sn}$ intrinsic diffusion and $\mathrm{Cu}-\mathrm{Sn}$ interdiffusion [28]................ 18

Figure 2-6 - Top: Examples of undesirable features formed on the surface during heat treatment of Cu coated with Sn. Bottom: SEM images of said features................................................ 20

Figure 2-7 - Plot of Equation 2.14 at different points in time. By reflecting around the vertical axis the same equation describes the case of finite body between two semi-infinite bodies. 29

Figure 2-8 - Analytical solution for diffusion on a composite medium. 32

Figure 3-1 - Stages a) I, II and III, b) IV and c) V. Lighter shades of gray represents a later point in time.

Figure 3-2 - Stages I, II and III for a layer of copper (left) and tin/liquid phase (right). Phase

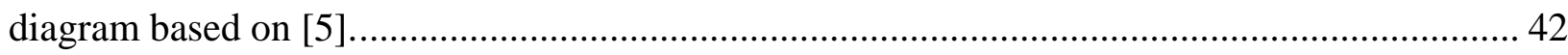

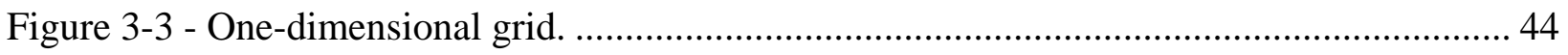

Figure 3-4 - One-dimensional grid showing the half control volume at a free boundary. .......... 49

Figure 3-5 - Error behavior at different values of $\Delta \mathrm{t}$ and $\Delta \mathrm{x}$ (continues on next page)............. 57

Figure 3-6 - a) Analytical curve superimposed by model approximation with $\Delta \mathrm{t}_{\max }$; b) Area under the curve of discrete analytical points, darker region shows where mass is being overestimated. c) Mass calculated from area under model approximation; d) detail showing analytical curve, and

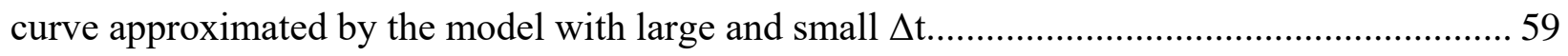

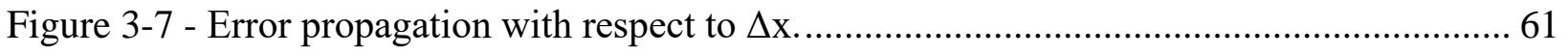

Figure 3-8 - Analytical and model solution for semi-infinite composite medium diffusion........ 63

Figure 3-9 - Harmonic average for D1 and D2 at different ratios. ....................................... 65 
Figure 3-10 - Moving boundary position at the end of stage 3 of heat treatment at $800{ }^{\circ} \mathrm{C}$ for each temperature profile. 67

Figure 3-11 - Concentration profile at the end of stage 3 of heat treatment at $800{ }^{\circ} \mathrm{C}$, every profile except 300s is superimposed. 67

Figure 3-12 - Moving boundary position at the end of stage 3 of heat treatment at $850{ }^{\circ} \mathrm{C}$ for each temperature profile. 69

Figure 3-13 - Concentration profile at the end of stage 3 of heat treatment at $850{ }^{\circ} \mathrm{C}$, constant temperature and 30s heating profiles are superimposed. 69

Figure 3-14 - Moving boundary position at the end of stage 3 of heat treatment at $900{ }^{\circ} \mathrm{C}$ for each temperature profile. 70

Figure 3-15 - Concentration profile at the end of stage 3 of heat treatment at $900{ }^{\circ} \mathrm{C}$, constant temperature and 30s heating profiles are superimposed. 70

Figure 3-16 - Moving boundary position during stages 2-4 for heat treatment at $800{ }^{\circ} \mathrm{C}$ with different heating rates. 72

Figure 3-17 - Moving boundary position during stages 2-4 for heat treatment at $850{ }^{\circ} \mathrm{C}$ with different heating rates. 72 Figure 3-18 - Moving boundary position during stages 2-4 for heat treatment at $900{ }^{\circ} \mathrm{C}$ with different heating rates. 73 Figure 3-19 - Composition profiles after solidification for different heat treatment temperatures. 73

Figure 3-20 - Time to solidification of different liquid amounts for different temperatures........ 75 Figure 3-21 - Time to solidification of different deposited amounts for different temperatures.. 75 
Figure 3-22 - Composition profiles of the same surface concentration obtained through different heat treatment temperatures. 77

Figure 3-23 - Voltammogram of sulfate tin plating bath.............................................................. 81

Figure 3-24 - Cross section BSE image of the bottom surface of sample \#1................................ 86

Figure 3-25 - Cross section BSE image of the top surface of sample \#1 ..................................... 86

Figure 3-26 - Experimental data (circles) and model simulation (line) of composition profile below eutectic layer of sample $\# 1$ heat treated at $850{ }^{\circ} \mathrm{C}$ for $60 \mathrm{~min}$. Simulation temperature $=860{ }^{\circ} \mathrm{C}$. 88

Figure 3-27 - Experimental data (circles) and model simulation (line) of composition profile on low tin content side of sample $\# 1$ heat treated at $850{ }^{\circ} \mathrm{C}$ for $60 \mathrm{~min}$. Simulation temperature $=860$ ${ }^{\circ} \mathrm{C}$. 88

Figure 3-28 - Experimental data (circles) and model simulation with concentration independent diffusion (line) of composition profile below eutectic layer of sample $\# 1$ heat treated at $850{ }^{\circ} \mathrm{C}$ for $60 \mathrm{~min}$. Simulation temperature $=860^{\circ} \mathrm{C}$. 90

Figure 3-29 - Experimental data (circles) and model simulation with concentration independent diffusion (line) of composition profile on low tin content side of sample $\# 1$ heat treated at $850{ }^{\circ} \mathrm{C}$ for $60 \mathrm{~min}$. Simulation temperature $=860^{\circ} \mathrm{C}$. 90

Figure 3-30 - Experimental data (circles) and model simulations (lines) of composition profile of sample \#2 heat treated at $820^{\circ} \mathrm{C}$ for $70 \mathrm{~min}$. 92

Figure 3-31 - Experimental data (circles) and model simulation (line) of composition profile of sample \#3 heat treated at $870{ }^{\circ} \mathrm{C}$ for 80 min. Simulation temperature $=880^{\circ} \mathrm{C}$. 92

Figure 3-32 - Experimental data (circles) and model simulation (line) of composition profile of sample \#4 heat treated at $800{ }^{\circ} \mathrm{C}$ for $70 \mathrm{~min}$. Simulation temperature $=820^{\circ} \mathrm{C}$. 94 
Figure 3-33 - Experimental data (circles) and model simulation (line) of composition profile of sample \#5 heat treated at $890{ }^{\circ} \mathrm{C}$ for $80 \mathrm{~min}$. Simulation temperature $=900{ }^{\circ} \mathrm{C}$. 94

Figure 3-34 - Possible range of composition profile estimated by model (gray area) and experimental composition profile obtained from specimen heat treated at $830{ }^{\circ} \mathrm{C}$ for 20 minutes. Simulation temperature $=840^{\circ} \mathrm{C}$. 96

Figure 3-35 - Top: optical image of surface of specimen heat treated at $810^{\circ} \mathrm{C}$ for $30 \mathrm{~min}$; average surface composition of each region as shown. Bottom: BSE image of cross-section stretched on vertical axis. Location of cross-section indicated by line on optical image 99 Figure 3-36 - Top: BSE cross-section image of tin-rich crater. Bottom: Composition profiles obtained from locations indicated on BSE image and simulated by model. 100 Figure 3-37 - Specimen heat treated at $450{ }^{\circ} \mathrm{C}$ for $15 \mathrm{~min}$. a) optical surface image; b) SE surface image; c) BSE cross-section image; d) higher magnification BSE cross-section image; e) EDS point scan forming composition profile of location shown on d). 102 Figure 3-38 - a) cross-section image from protrusion of specimen heat treated at $450{ }^{\circ} \mathrm{C}$ for 15 min. b) Composition range predicted (gray area) and measured composition profile (circles) of sample heat treated at $450{ }^{\circ} \mathrm{C}$ for $15 \mathrm{~min}$ followed by $830^{\circ} \mathrm{C}$ for $23 \mathrm{~min}$. 103 Figure 3-39 - a) specimen heat treated at $450{ }^{\circ} \mathrm{C}$ for $15 \mathrm{~min}$. b) same specimen as in "a” heat treated at $830^{\circ} \mathrm{C}$ for $23 \mathrm{~min}$. 104

Figure 3-40 - a) surface optical image of sample heat treated at $250{ }^{\circ} \mathrm{C}$; b) surface SEM image of one of the protrusions from sample on a); c) cross-section SEM image showing copper covering surface layer; d) cross-section BSE image of protrusion 106 Figure 3-41 - a) specimen heat treated at $25{ }^{\circ} \mathrm{C}$ for $30 \mathrm{~min}$; b) same specimen after heat treatment at $830^{\circ} \mathrm{C}$ for $20 \mathrm{~min}$. 107 
Figure 3-42 - Typical load-displacement curve obtained during nanoindentation testing. ........ 110

Figure 3-43 - Nanoindentation hardness test results for different surface compositions. .......... 110

\section{List of Tables}

Table 2-1 - Standard reduction-oxidation potentials for Sn [30] ......................................... 24

Table 3-1 - Composition of stannous sulfate acidic electrolyte [1] ...................................... 79

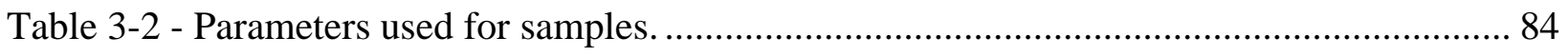

\section{Abbreviations}

FCC - Face Centered Cubic

BCC - Body Centered Cubic

BCT - Body Centered Tetragonal

SEM - Scanning Electron Microscope

EDS - Energy Dispersive Spectroscopy

SE - Secondary Electron

BSE - Backscattered Electron 


\section{Chapter 1 Introduction}

\subsection{Background}

As the field of materials engineering develops, new and complex alloys are introduced to improve the operation of various machines. However, it is not unusual to encounter the situation in which a single material does not have all of the necessary properties. Often, the surface of a component and its core requires distinct materials each with unique properties, in order for the component to function as required. Properties such as hardness, wear and corrosion resistance and even appearance can be improved at the surface level to make service possible or economically viable through the use of surface engineering. These improvements can be obtained metallurgically, mechanically, chemically or through the application of coatings [1].

Among the commercial metals, the production and consumption of copper and its alloys are particularly high, behind only steel and aluminum [2], thus making these an interesting target of research. Bronze is a popular alloy of copper $(\mathrm{Cu})$ and tin $(\mathrm{Sn})$ due to its corrosion and wear resistance and golden appearance (in its $\alpha$ phase), but the formation of the correct phase as a coating may prove to be challenging. Electrodeposition is a widely used method for producing coatings as it is capable of depositing thicknesses that range from single to hundreds of micrometers. However, the most frequently used electrolytes for direct bronze deposition are highly toxic [3] and more than one phase can be deposited during the process [4], thus altering the resultant surface properties. An alternative to the direct electrodeposition of bronze is the separate deposition of $\mathrm{Cu}$ and $\mathrm{Sn}$, followed by a heat treatment process to obtain a bronze finish on the surface through diffusion. 
The solid state diffusion of $\mathrm{Cu}$ and $\mathrm{Sn}$ has a limited temperature range due to the low melting point of Sn $\left(232^{\circ} \mathrm{C}\right)$ and is not suitable for obtaining an $\alpha$ bronze phase mainly for two reasons: its saturated composition is very low at this temperature range $(<1 \mathrm{Sn}$ atomic percent (at.\%)) and intermetallic phases obstruct the process. Above the melting temperature of Sn lies a wide temperature range where the diffusion of liquid Sn /solid Cu can occur to obtain an $\alpha$ bronze phase, although many other phases lies between them. For this reason, high temperature sections $\left(>800^{\circ} \mathrm{C}\right)$ are of particular interest as no other phases are present, and the liquid phase immediately forms $\alpha$ solid bronze. During diffusion at elevated temperatures, the material undergoes many different stages that involve the melting of $\mathrm{Sn}$, substrate $(\mathrm{Cu})$ diffusion induced melting, solidification and solid state diffusion. The occurrence of these stages causes the diffused composition profile of Sn to differ considerably from that of a simple solid state diffusion, thus adding much complexity to the problem. Every parameter involved (e.g., thickness of Sn coating, heat treatment temperature, time) greatly affects the resultant phase and composition of the surface, which leads to different properties. Another aspect of diffusion often disregarded, or rather, simplified, is the dependency of the diffusion coefficient on the local composition.

As mentioned above, there are many aspects involved when trying to predict the resultant surface composition of a Cu-Sn system at high temperatures, be it the complexity of the process or different variables that affect the result. Situations such as these are ideal for the application of numerical modeling to simulate physical behavior so as to predict the results of the process. Additionally, a secondary problem exists parallel to obtaining the correct surface composition: the resultant surface is often riddled with small circular depressions on the surface which display a higher Sn content. These Sn-rich craters are undesirable as their presence reduces the aesthetics and their different concentrations and microstructure change the surface properties. 


\subsection{Objective}

This work aims to predict the surface composition profile of a $\mathrm{Cu}-\mathrm{Sn}$ diffusion couple that is heat treated at temperatures above $800^{\circ} \mathrm{C}$. In order to do so, the main objective of this research is to properly elucidate the physical processes involved during the diffusion heat treatment and, based on those mechanisms, build a numerical model to predict the influence of processing parameters and simulate the resultant composition profile.

\subsection{Work Done}

After careful interpretation of the $\mathrm{Cu}-\mathrm{Sn}$ phase diagram, the behavior of the $\mathrm{Cu}-\mathrm{Sn}$ couple during the entire heat treatment process is better understood. Consequently, a one dimensional finite difference numerical model has been developed through the discretization of Fick’s laws of diffusion and Stefan's condition to express a liquid/solid moving boundary. The boundary conditions are applied in accordance with the description of the physical process. The model is capable of predicting the composition profile of Sn by using the heat treatment temperature; heating rate; thickness of the deposited Sn; and heat treatment duration as the parameters. Model validation is done by comparing the simulated composition profiles with experimental cross section profiles of the heat treated samples. Nanoindentation hardness testing indicates an increase in hardness, and consequently wear resistance as the Sn content on the surface increases, as expected. Cross sectional analysis of Sn-rich craters found on the surface of heat treated samples is carried out with the proposed model to present a cause and solution for this surface feature.

\subsection{Major Findings}

The developed numerical model is capable of simulating the behavior of a $\mathrm{Cu}-\mathrm{Sn}$ couple during heat treatment. The large deviation obtained between the simulations of constant diffusion coefficient and concentration dependent diffusion coefficient shows that, for this system, the 
simplification of an independent diffusion coefficient, which is a common phenomenon, is not acceptable. The validation of the model with the experimental results shows good correlation with the simulated predictions, thus serving as corroboration for the physical process expressed. When the aim is to obtain a high Sn content on the surface, the proposed model shows that with the temperature range investigated, lower temperatures are capable of: higher concentrations due to the solid saturation concentration; faster complete solidification; and obtaining a composition profile where a higher Sn content is maintained close to the surface at the expense of the depth of the diffusion.

The surface of heat treated samples contained many Sn-rich craters. Investigation of said craters shows that their formation is likely caused by the agglomeration of liquid Sn as the melting temperature is reached. These bubbles of liquid phase lead to increased substrate dissolution through diffusion, which creates craters that can accommodate an excessive liquid phase, which remains liquid at the end of the heat treatment process. The deliberate formation of an intermetallic layer through heat treatment at low temperatures creates a barrier to the formation of craters, thus eliminating this problem.

\subsection{Thesis Layout}

This dissertation contains 4 chapters, organized as follows:

$>$ Chapter 1 provides background information, objective of the research, the work done and major findings from the study;

> Chapter 2 provides a review of the literature focusing on the phases of the $\mathrm{Cu}$-Sn system, the diffusion mechanisms and its mathematical approximations, electrodeposition as a method of coating and the aspects involved on building a diffusion model; 
$>$ Chapter 3 provides a description of the expected behavior during heat treatment, a discretization methodology of the pertinent equations, as well as the methodology, equipment and materials used to carry out the experimental verification of the proposed model;

$>$ Chapter 4 provides conclusions for the work done and suggestions for future work. 


\section{Chapter 2 Literature Survey}

\subsection{Copper-Tin System}

$\mathrm{Cu}$ is a metal with a face centered cubic (FCC) crystallographic structure. On the Cu-Sn system, this crystal structure is retained with low amounts of Sn as the denominated $\alpha$-phase, often represented as (Cu). The solubility of $\mathrm{Sn}$ in the $\alpha$-phase is virtually null at low temperatures, but this value increases as temperature is increased until it peaks at $518^{\circ} \mathrm{C}$, in which the solubility reaches 9.1 Sn at.\%. After this point, further increases in temperature will reduce the solubility of Sn in the $\alpha$ phase, which reaches zero at the melting temperature of pure $\mathrm{Cu}, 1084.87^{\circ} \mathrm{C}$ [5].

The $\beta$-phase, formed by a body centered cubic (BCC) crystal structure, is separated from the $\alpha$-phase by a two phase field between $566^{\circ} \mathrm{C}$ and $798^{\circ} \mathrm{C}$. At its highest temperature, the $\beta$-phase has the least amount of dissolution of Sn, with $12.6 \mathrm{Sn}$ at.\%. The maximum solubility of Sn in the $\beta$-phase is difficult to determine as this phase undergoes an ordering phase transformation at around $17 \mathrm{Sn}$ at.\%, thus transforming into the $\gamma$-phase which has a D0 3 type structure in the FCC space group. Until recently, it was believed that a two phase field existed between the $\beta$ and $\gamma$ phases, as can be seen in various versions of $\mathrm{Cu}-\mathrm{Sn}$ phase diagrams. This was mainly due to the observation of thermal peaks in this region during differential thermal analysis. Li et al. [6] proposed that the small peak observed is due to a higher order phase transformation rather than a two phase region. The $\gamma$-phase is present throughout a large range of Sn content, forming roughly between $16.5 \sim 29.0 \mathrm{Sn}$ at.\%, and large range of temperatures, between $518^{\circ} \mathrm{C}$ and $758^{\circ} \mathrm{C}$. Both the $\beta$ and $\gamma$ phases cannot be found at lower temperatures, since quenching will result in transformation into either a lower temperature or metastable phases [5]. 
Four intermetallic phases can be found in this system: $\delta, \zeta, \varepsilon$ and $\eta$. The $\delta$-phase is often formed from eutectoid reactions that involve the $\beta, \gamma, \varepsilon, \delta$ or $\zeta$ phases. It is composed of a cubic crystal structure that follows the stoichiometric phase $\mathrm{Cu}_{41} \mathrm{Sn}_{11}$, at $20.8 \mathrm{Sn}$ at.\% between $350^{\circ} \mathrm{C}$ and $603^{\circ} \mathrm{C}$. The $\zeta$-phase lies between the $\delta$ and $\varepsilon$ phases in terms of Sn content, with 22 Sn at.\%. It is the last phase identified in the Cu-Sn system and its crystal structure was determined in 1936 by Haase [7]. The structure, which is formed between $589^{\circ} \mathrm{C}$ and $641^{\circ} \mathrm{C}$, follows a hexagonal configuration and the stoichiometric $\mathrm{Cu}_{10} \mathrm{Sn}_{3}$ phase. The $\eta$ and $\varepsilon$ intermetallic phases can form at the lower ranges of temperatures of this system, thus making them present in applications such as soldering of electronic devices. With the recent call to eliminate lead $(\mathrm{Pb})$-based solder [8]-[11], these intermetallics have thus been the focus of many researchers.

The $\eta$-phase forms at temperatures below $408^{\circ} \mathrm{C}$. At a temperature range of $186^{\circ} \mathrm{C} 189^{\circ} \mathrm{C}$ this phase undergoes an ordering transformation, becoming ordered $(\eta ')$ below this temperature range. Its ordered configuration ( $\left.\eta^{\prime}\right)$ is formed by a monoclinic crystal structure; with disordered $(\eta)$, it is formed by a hexagonal crystal structure. Both cases follow the stoichiometric $\mathrm{Cu}_{6} \mathrm{Sn}_{5}$ phase and are formed between 43.4 and $44.3 \mathrm{Sn}$ at.\%. This intermetallic has been reported to form between $\mathrm{Cu}-\mathrm{Sn}$ couples at temperatures as low as $-2^{\circ} \mathrm{C}$ [12], [13]. When formed in a Cu-Sn couple, its interface with $\mathrm{Sn}$ is highly non-planar, but remains uniform on the Cu side [14]. Gagliano et al. [15] analyzed the nucleation kinetics of $\eta$ by dipping Cu coupons into molten Sn, and demonstrated the quick formation of $\eta$ crystallites, which appeared even when the dipping was carried out for a single second.

The $\varepsilon$-phase has the second highest temperature range in this system (after the $\alpha$-phase), with its higher limit at $676^{\circ} \mathrm{C}$ and has been reported to form at temperatures as low as $60^{\circ} \mathrm{C}$ [13]. It has an orthorhombic crystal structure and follows the stoichiometric phase Cu3Sn. During the heat 
treatment of Cu-Sn couples $\left(\sim 200^{\circ} \mathrm{C}\right)$, a layered system of the $\mathrm{Cu} / \varepsilon / \eta / \mathrm{Sn}$ form is reported, where the $\varepsilon$-phase is the last to form but grows quickly by consuming the $\eta$-phase. Similar to what has been observed in $\eta, \varepsilon$ retains a planar geometry on the $\mathrm{Cu}$ interface but is dissimilar on its interface with $\eta[12]-[14],[16]$.

Finally, Sn can assume two different phases, $\alpha \mathrm{Sn}$ and $\beta \mathrm{Sn}$. $\alpha \mathrm{Sn}$ has a diamond cubic structure and can be formed at temperatures below $13^{\circ} \mathrm{C}$, with a negligible $\mathrm{Cu}$ solubility [17]. Above this temperature, the $\beta$ Sn phase is formed, until Sn reaches its melting temperature $\left(232^{\circ} \mathrm{C}\right)$. Unlike the convention for the $\mathrm{Cu}$ side, $(\mathrm{Sn})$ is often the designation given to the $\beta$ phase of $\mathrm{Sn}$, rather than its $\alpha$ phase. With a body centered tetragonal (BCT) crystal structure, $\beta S n$ has a maximum $\mathrm{Cu}$ solubility of $1.3 \mathrm{Cu}$ at.\%, which reduces its melting temperature to $227^{\circ} \mathrm{C}$.

The most recent phase diagram, proposed in 2013 by Fürtauer et al. [5] and Li et al. [6], is shown in Figure 2-1. In their work, thermal, metallographic and crystallographic analyses were conducted with special emphasis on the region that has less than 30 Sn at.\%.

\subsection{Diffusion}

The behaviour of materials, that is, how their properties react to external stimuli, can be attributed to changes in their microstructure. These changes are most often dictated by diffusion, which defines the extent and rate of said processes. Therefore, whether it is oxidation, precipitation or homogenization, understanding diffusion is crucial for predicting the performance of materials.

The problem of determining the kinetics of diffusion processes does not need to be related to the atomic structure involved. The material can be treated as a continuum and the process can be expressed with a differential equation [18]. 


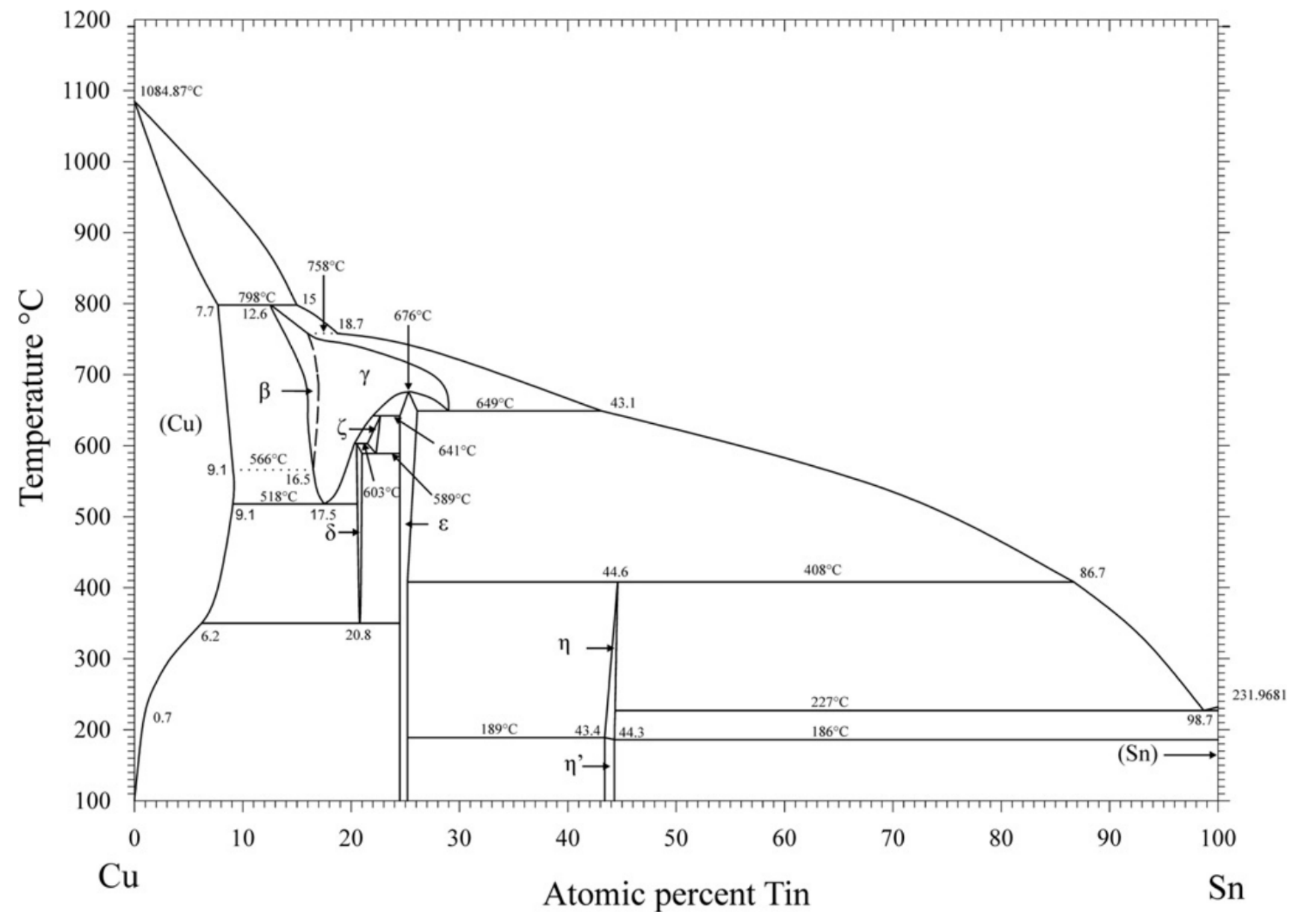

Figure 2-1 - Cu-Sn phase diagram [5], [6]. 


\subsubsection{Diffusion Equations}

Atoms are constantly oscillating around the equilibrium position, but every so often, an atom will reach the excited state, which means that it will have enough energy to move from its location to a neighboring vacant site. By summing the number of atoms per unit time that go through a particular cross sectional area, the net flux of atoms can be expressed by using the following equation:

Equation 2.1

$$
J=-\frac{1}{6} \Gamma \alpha^{2}\left[\frac{\partial C}{\partial x}\right]_{t}
$$

where $J$ is the flux, with units of mass per length squared times time, $\Gamma$ is the atom jump frequency, $\alpha$ is the distance between the atom planes, $C$ is the concentration of the diffusing substance and $x$ is distance. The fraction in the equation comes from the fact that in a simple cubic lattice, an atom can jump in any of the 6 different directions, but only one is of interest. The ratio of the flux and concentration gradient is defined as the diffusion coefficient.

Equation 2.2

$$
D \equiv \frac{-J}{\left[\frac{\partial C}{\partial x}\right]_{t}}=\frac{1}{6} \Gamma \alpha^{2}
$$

An increase in temperature would increase the atom jump frequency, thus increasing the diffusion coefficient. This relation is found to be exponential, as represented by the empirical Arrhenius equation [19]:

Equation 2.3

$$
D=D_{0} e^{-\frac{Q}{R T}}
$$


where $D_{0}$ is a pre-exponential coefficient often referred to as the frequency factor, $Q$ is the activation energy, $R$ is a gas constant and $T$ is the absolute temperature. The accuracy of this empirical relation can be assessed by plotting the natural logarithm of the diffusion coefficient with respect to the reciprocal of temperature. If the plotted results come close to a straight line, this indicates good correlation, and from it, the values of activation energy and frequency factor can be obtained by the slope and intersecting of the curve, respectively.

By replacing the diffusion coefficient back into Equation 2.1:

\section{Equation 2.4}

$$
J=-D\left[\frac{\partial C}{\partial x}\right]_{t}
$$

It should be noted that $t$ in the above equation denotes that the calculated flux corresponds to that particular moment. Should the concentration gradient change, the flux would also change accordingly. Consequently, this is a steady-state equation and is entitled Fick's first law of diffusion per Adolf Fick [20].

When time is a variable, namely in a non-steady-state or transient condition, Equation 2.4 is still valid, but impractical. A second differential equation can be subsequently obtained from time as a variable, which is Fick`s second law of diffusion:

\section{Equation 2.5}

$$
\frac{\partial c}{\partial t}=\frac{\partial}{\partial x}\left(D \frac{\partial C}{\partial x}\right)
$$

Both equations apply towards ideal solutions (thermodynamic activity coefficient equals to unity) in an isotropic medium. Furthermore, the driving force for diffusion (free energy gradient) is considered to be purely due to a concentration gradient, as opposed to other gradients such as gravitational, thermal, pressure, etc. [21]. 
The diffusion coefficient is often simplified to be independent of every other variable in the system. When this approximation is applied, Equation 2.5 can be solved for different initial and boundary conditions, usually by using a series of error functions or related integrals. For some situations this is reasonable, but realistically, in most systems, the diffusion coefficient will vary with concentration and its variance is sizeable [22].

\subsubsection{Diffusion Mechanisms}

Unlike liquids or gasses, the structure of solids greatly restricts the movement of their atoms, thus requiring certain conditions, or mechanisms, for diffusion to occur. The most commonly encountered modes of diffusion in solids occur through both interstitial and vacancy mechanisms, which take place due to defects in the crystal structure.

\subsubsection{Interstitial Mechanism}

It is convenient to imagine atoms as spheres when trying to understand the structure of materials. The lattice and interstitial positions can be conceptually illustrated by having every sphere touch its neighbours, where lattice sites are those occupied by spheres (atoms) and interstitial sites by the empty spaces between the spheres. The interstitial mechanism of diffusion consists of an interstitial atom moving (diffusing) through the material by jumping between the interstitial sites (atom “a” in Figure 2-2).

The energy required for this diffusion process is related to the effort necessary for the interstitial atoms to "squeeze" past the atoms in the lattice site on their way to a vacant interstitial site. 


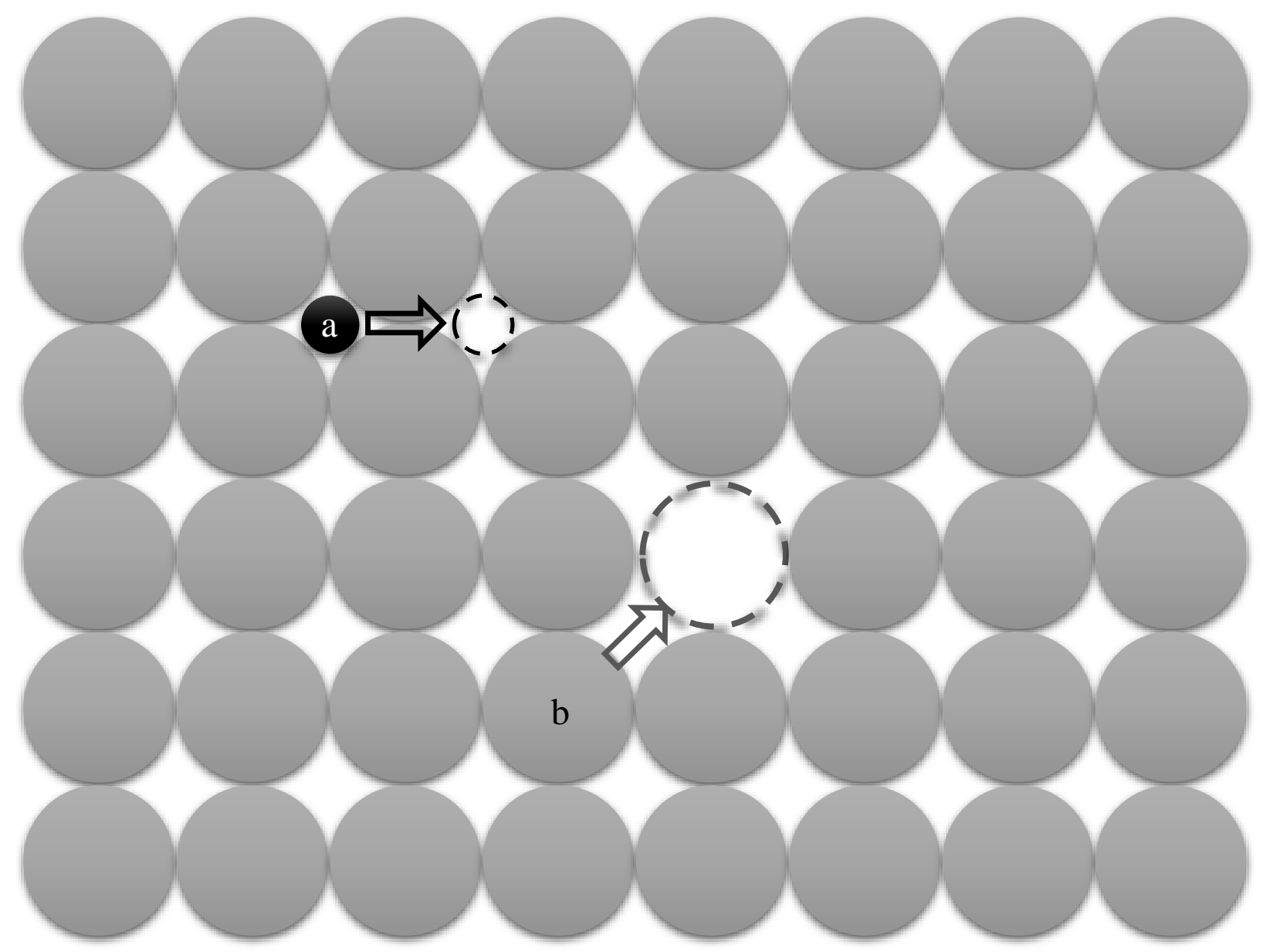

Figure 2-2 - Lattice sites (occupied by gray spheres) and interstitial sites (white space between spheres or location of black atom). Diffusion through interstitial and vacancy mechanisms are illustrated by the movement of atoms " $a$ " and " $b$ ”, respectively. 


\subsubsection{Vacancy Mechanism}

Interstitial atoms are often found in very dilute solutions, and thus the probability of a vacant neighboring interstitial site is very high. However, atoms that diffuse through lattice sites need to diffuse into an empty lattice space, known as vacancy, which are much less common. Vacancies are point defects, and as such, increase the instability of the material. However, a low, but nonzero, amount of vacancies (which increases with temperature) are required for the stability of the matrix. For lattice atoms to diffuse, not only do they require enough energy to "squeeze" past a row of atoms, but a neighboring lattice site also needs to be vacant. For this reason, vacancy diffusion is usually orders of magnitude slower than interstitial diffusion.

\subsubsection{Types of Diffusion Coefficients}

So far, diffusion coefficients have been treated as a single variable, but can actually be classified as self-diffusion, intrinsic diffusion and interdiffusion. Self-diffusion is the random movement of atoms in the absence of a concentration gradient, which is usually found in pure metals. Intrinsic diffusion is the movement of one of the alloy elements in an alloy; each element will have its own intrinsic diffusion coefficient value. In a substitutional alloy, in which alloying elements occupy lattice sites, the intrinsic diffusion coefficient of each component is an important aspect of the overall diffusion of the system, and each will react to changes in the temperature and alloy composition. Finally, the interdiffusion coefficient is the actual diffusion resultant from the movement of every element in an alloy. It can be approximated by the following equation, for a binary alloy [23]:

Equation 2.6

$$
\widetilde{D}\left(X_{1}\right)=\tilde{V}_{1} D_{1} X_{2}+\tilde{V}_{2} D_{2} X_{1}
$$


where $\widetilde{D}$ is the interdiffusion coefficient, and $\widetilde{\mathrm{V}}_{\mathrm{x}}, D_{x}$ and $X_{x}$ are the partial molar volume, intrinsic diffusion coefficient and atomic fraction, respectively, of the two components in the alloy. One interesting implication that comes from the aforementioned equation is that as the atom fraction of one element approaches zero, which is the case in dilute alloys, the interdiffusion coefficient approaches that of the dilute element, rather than the abundant element.

\subsubsection{Copper and Tin Diffusion}

Butrymowicz et al. [24] compiled over 30 years' worth of data on the self-diffusion of $\mathrm{Cu}$. They regarded the work by Rothman and Peterson [25] to have the most accurate measurement, in which the activation energy of $Q=50.5 \mathrm{kcal} / \mathrm{mol}$ and frequency factor of $D_{0}=0.78 \mathrm{~cm}^{2} / \mathrm{s}$ were obtained. Their experiments were done on a single crystal $\mathrm{Cu}$ and purified $\mathrm{Cu}-67$ tracer, at the temperature range of $700^{\circ} \mathrm{C}$ to $1060^{\circ} \mathrm{C}$.

Fensham [26] investigated the self-diffusion coefficients in solid Sn close to but below its melting temperature. It was reported that due to its tetragonal structure, the diffusion coefficients are highly anisotropic. The ratio of the diffusion coefficient value that is parallel and perpendicular to the c-axis varies between 2 , at $180^{\circ} \mathrm{C}$, and 3 , at $223^{\circ} \mathrm{C}$. The activation energy and frequency factor were calculated as $Q=10.5 \mathrm{kcal} / \mathrm{mol}$ and $D_{0}=1.20 \times 10^{-5} \mathrm{~cm}^{2} / \mathrm{s}$ for the c-axis and $Q=$ $5.9 \mathrm{kcal} / \mathrm{mol}$ and $D_{0}=3.70 \times 10^{-8} \mathrm{~cm}^{2} / \mathrm{s}$ for the a-axis.

At higher temperatures, where Sn assumes its liquid phase, diffusion increases considerably, as expected from the increase in atomic mobility. Bruson and Gerl [27] measured the diffusivity of $\mathrm{Sn}^{113}$ tracer, among other metals, in liquid Sn, from its melting temperature up to $1925 \mathrm{~K}$ $\left(1652^{\circ} \mathrm{C}\right)$. No value for activation energy or frequency factor was reported, but from the self- 
diffusion coefficients provided, it is possible to construct an Arrhenius plot and obtain the following: $Q=3.1 \mathrm{kcal} / \mathrm{mol}$ and $D_{0}=3.8 \times 10^{-4} \mathrm{~cm}^{2} / \mathrm{s}$.

To obtain a sense of scale, the self-diffusion coefficients of $\mathrm{Sn}$ and $\mathrm{Cu}$ are plotted in Figure 2-3. It can be seen that the self-diffusion of $\mathrm{Sn}$ is orders of magnitude faster than that of $\mathrm{Cu}$.

Hoshino et al. [28] investigated the interdiffusion and intrinsic diffusion of $\mathrm{Cu}-\mathrm{Sn}$ solid solutions between 1 and $6 \mathrm{Sn}$ at.\% at a temperature range between $700^{\circ} \mathrm{C}$ and $830^{\circ} \mathrm{C}$. Their results for the interdiffusion activation energy and frequency factor for various concentrations are plotted in Figure 2-4. The interdiffusion activation energy at $4 \mathrm{Sn}$ at.\% shows a sudden drop compared to the values obtained for higher and lower concentrations. Hoshino et al. [28] attributed this local minima to a probable variation in the electronic structure of the alloy.

The interdiffusion coefficients were determined by analyzing the movement of Kirkendall markers in semi-infinite diffusion couples. In every couple analyzed, the markers shifted towards the Sn-rich side, thus indicating that $\mathrm{Sn}$ is the faster diffusing element in the alloy. This behaviour was confirmed by determining the intrinsic diffusion coefficients which showed that the intrinsic diffusion of $\mathrm{Sn}$ is approximately three times that of $\mathrm{Cu}$ and the logarithm of both intrinsic diffusivities increase linearly with the Sn content, as can be seen in Figure 2-5. It can also be seen that, as mentioned before, the interdiffusion coefficient of the alloy in this concentration range (low Sn concentrations) approaches the value for the intrinsic diffusion of Sn. 


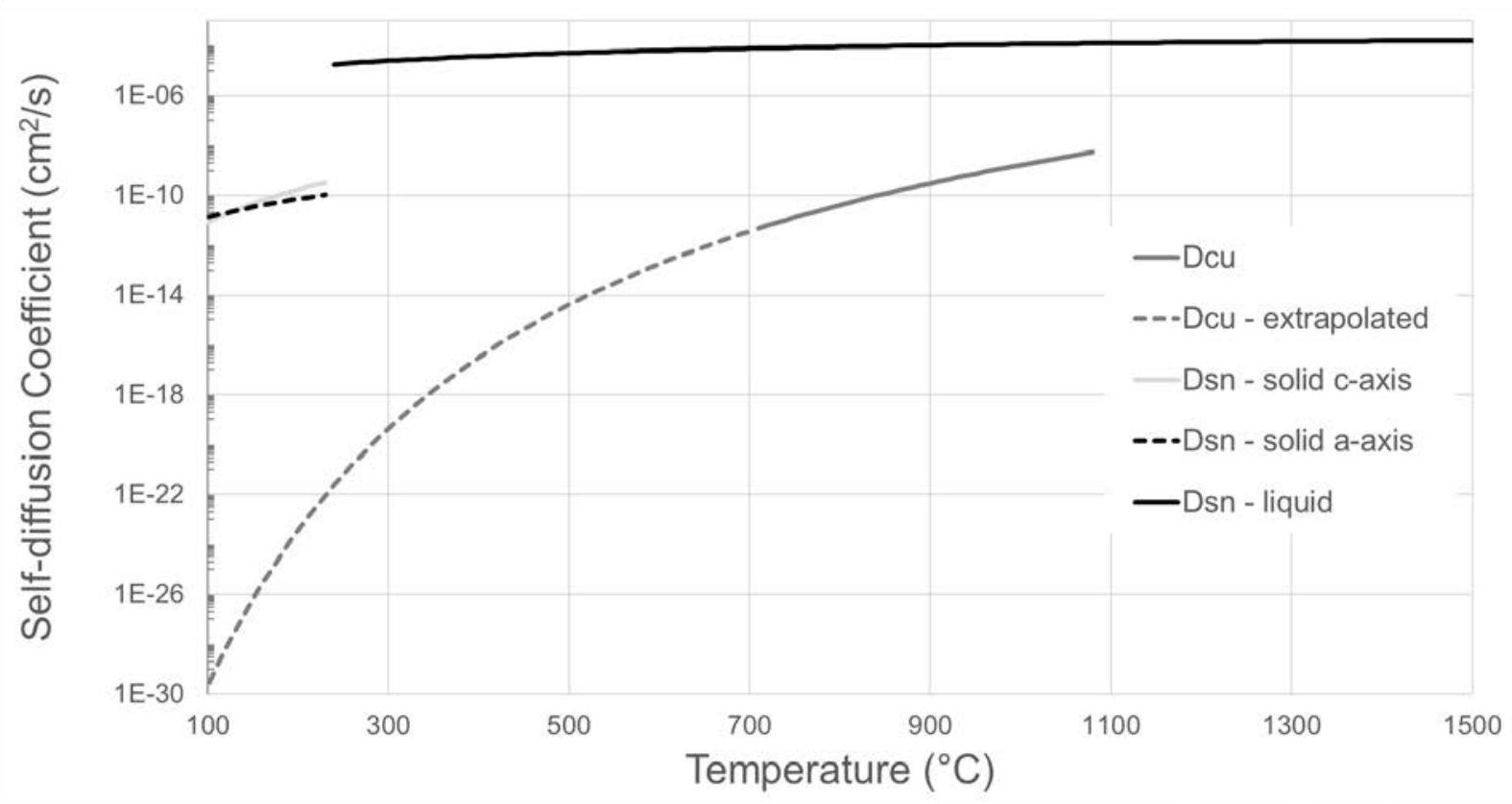

Figure 2-3 - Self-diffusion coefficients of solid Cu [25] and Sn (c and a-axis) [26] and liquid Sn [27].

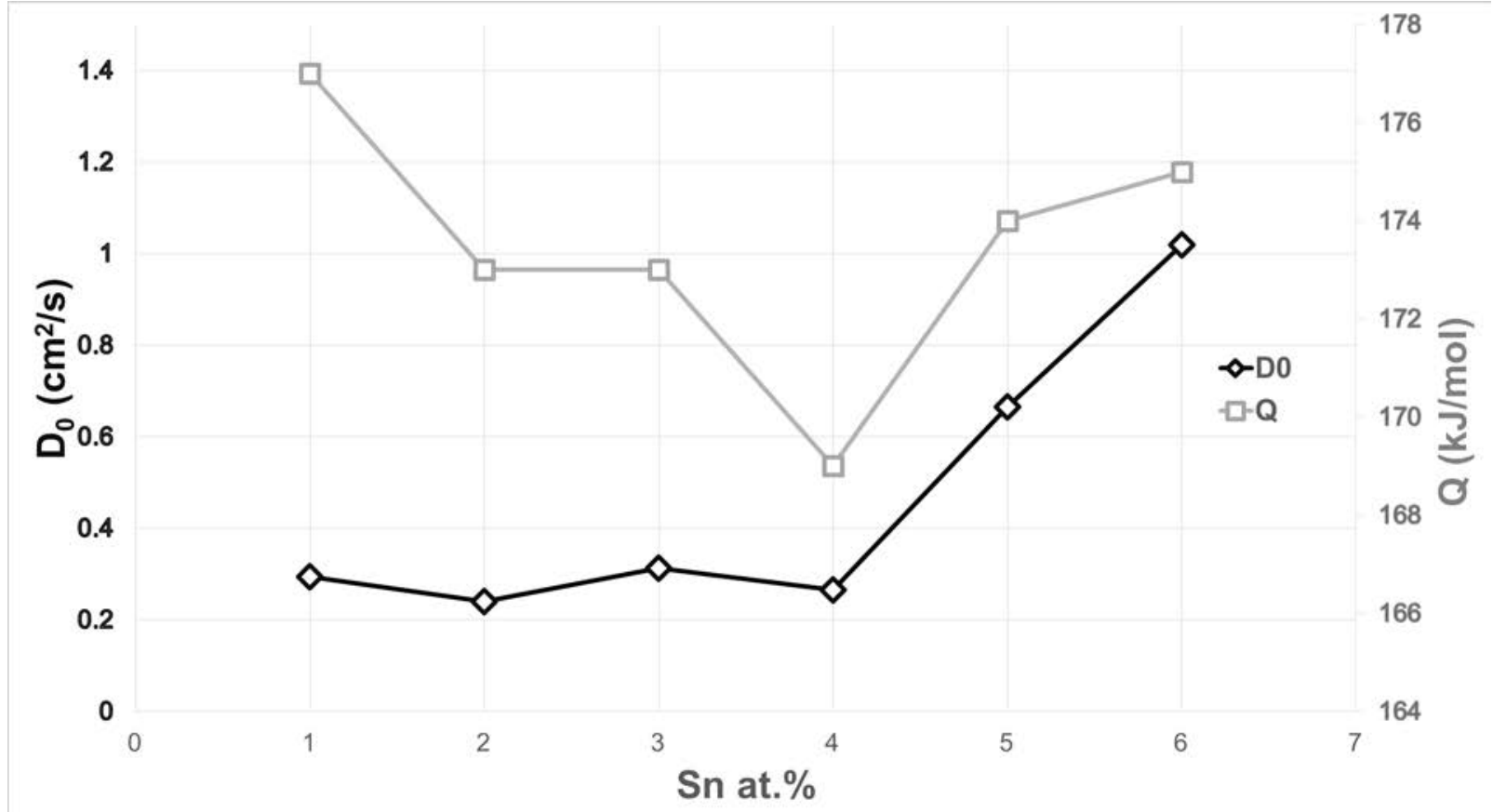

Figure 2-4 - Interdiffusion activation energy (right axis) and frequency factor (left axis) as a function of Sn at.\% [28]. 


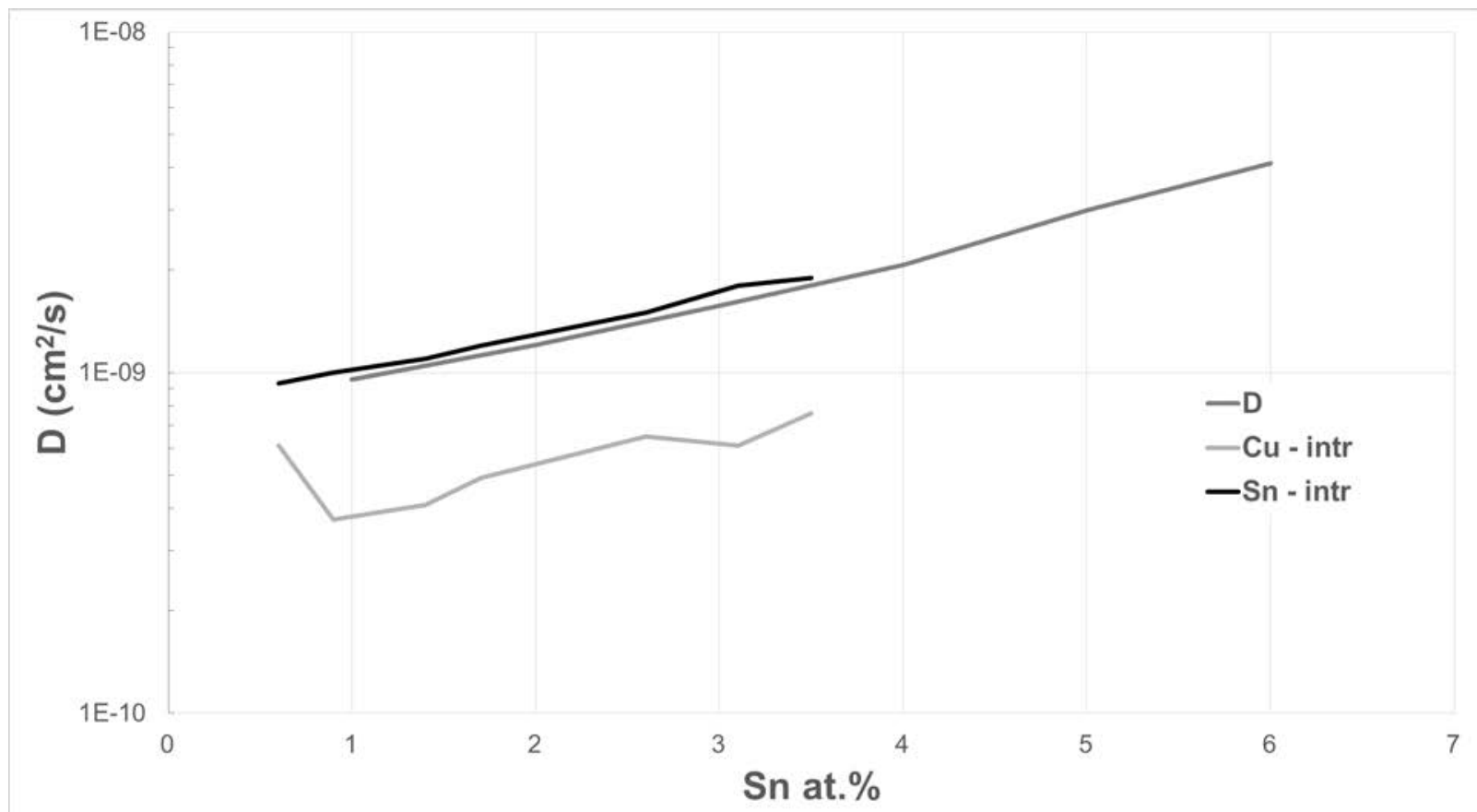

Figure 2-5 - Values for Cu and Sn intrinsic diffusion and Cu-Sn interdiffusion [28]. 


\subsection{Sn-rich Craters}

Information available in the literature on the heat treatment of $\mathrm{Cu}$ and $\mathrm{Sn}$ at high temperatures as a surface coating is limited. The majority of publications either analyze the formation of bronze by the complete melting of the elements; heat treatment of bulk $\mathrm{Sn}$ and $\mathrm{Cu}$ in diffusion studies; or study intermetallic formation at low temperatures with focus on electronic soldering.

When a $\mathrm{Cu}$ specimen covered with a layer of $\mathrm{Sn}$ is heat treated to form $\alpha$ bronze on the surface, the anticipated smooth surface is instead populated by shallow craters, composed of a higher content of Sn compared to the surrounding areas of the surface. These craters usually have a circular shape but their overall size, morphology and population may vary even with the use of constant parameters in the heat treatment. Examples of this phenomenon are shown in Figure 2-6.

The presence of these craters is irrefutably undesirable as it is detrimental for every property aspired by the surface treatment. 

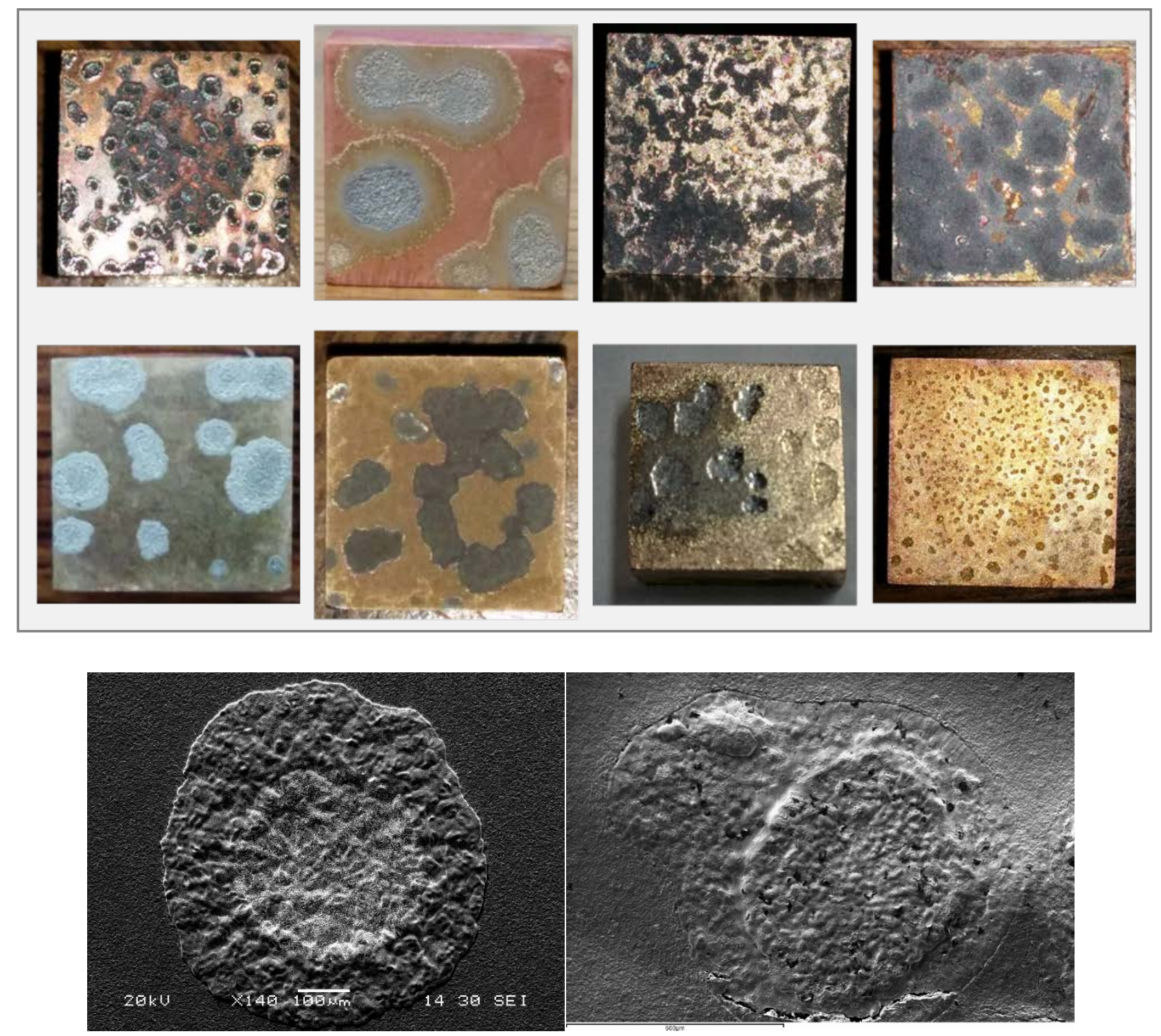

Figure 2-6 - Top: Examples of undesirable features formed on the surface during heat treatment of $\mathrm{Cu}$ coated with Sn. Bottom: SEM images of said features. 


\subsection{Electrodeposition}

Electrodeposition is a film growing process often used in surface treatments due to its capability of depositing, with a fairly high precision, thin metallic layers of a wide range of thickness and grain sizes.

There are many different techniques that vary according to the type of material that is being deposited, the desired deposition properties and even the receiving substrate. Due to the scope of this study, the deposition of Sn onto $\mathrm{Cu}$ will be used to simply explain the electrodeposition process.

The cathode, $\mathrm{Cu}$, and anode, $\mathrm{Sn}$, are placed in an electrolyte solution that contains Sn ions. $\mathrm{Cu}$ is supplied with electrons from a power source, which attract the $\mathrm{Sn}$ ions, thus depositing them onto its surface. Meanwhile, the anode, a Sn plate, is connected to the positive pole of the power source. Depleted of electrons, the Sn plate supplies new ions to the electrolyte. The reactions that result from the electrodeposition can be characterised by using the following equations:

Equation 2.7

$$
\text { Reduction or cathodic reaction: } \quad \mathrm{Sn}^{2+}+2 e^{-} \rightarrow \mathrm{Sn}
$$

Equation 2.8

$$
\text { Oxidation or anodic reaction: } \quad S n \rightarrow S n^{2+}+2 e^{-}
$$

The rate of deposition can be estimated by using Faraday's law of electrolysis, as shown in the following equation:

Equation 2.9

$$
m=\frac{Q M}{F Z}
$$


where $m$ is mass, $Q$ is the total charge in coulombs, $M$ is molar mass, $F$ is Faraday's constant (96485 [C/mol]) and $z$ is the valence number of the ion.

By replacing mass with density multiplied by volume, substituting the total charge with current multiplied by time, and converting volume into surface area multiplied by thickness, the deposited thickness can be expressed by:

Equation 2.10

$$
d=\frac{I t M}{F z \rho A}
$$

where $d$ is the deposited thickness, $I$ is the current, $\rho$ is the density of the element being deposited and $A$ is the surface area of the cathode.

The importance of Equation 2.10 is evident as it relates deposition thickness, which is the goal of electrodeposition, with the cathode area, a known variable, and current, one of the main parameters of the process. For generalization purposes, the current and surface area are often combined into current density i $\left[\mathrm{A} / \mathrm{cm}^{2}\right]$, which is independent of the sample size or shape.

Equation 2.10 can be further rewritten to define three convenient factors: the volume electrochemical equivalent, $\mathrm{K}_{\mathrm{v}}\left[\mathrm{cm}^{3} / \mathrm{C}\right]$, which expresses the volume deposited for a given current density and time; the mass equivalent, $\mathrm{K}_{\mathrm{m}}$ [g/Ah], which, similarly, expresses the mass deposited; and the one-dimensional equivalent, $\mathrm{K}_{1}\left[\mu \mathrm{m} /\left(\mathrm{Ah} / \mathrm{dm}^{2}\right)\right.$, which, for thin coatings, gives the thickness deposited in $[\mu \mathrm{m}]$. The values of $\mathrm{K}_{\mathrm{v}}, \mathrm{K}_{\mathrm{m}}$ and $\mathrm{K}_{\mathrm{l}}$ for the deposition of Sn are 0.3033, 2.214 and 30.33, respectively [29].

\subsubsection{Potential}

When a metal is immersed into a solution of its own ions, as is the case during the deposition process, an equilibrium reduction-oxidation (redox) reaction is established. Since it is at 
equilibrium, the rate that the metal ions gain electrons and deposit on the surface of the metal is the same as the rate that the metal atoms lose electrons and leave the surface as ions. The half-cell potential associated with this reaction can be calculated by using the Nernst equation:

Equation 2.11

$$
E=E_{0}+2.302585 \frac{R T}{z F} \log \frac{a_{o x}}{a_{r e}}
$$

where $\mathrm{E}$ is the half-cell potential, $\mathrm{E}_{0}$ is the standard half-cell potential, $\mathrm{R}=8.314462\left[\mathrm{~J} \mathrm{~K}^{-1}\right.$ $\mathrm{mol}^{-1}$ ] is a gas constant, $\mathrm{T}$ is the absolute temperature and aox and are are the activities of the oxidized and reduced species respectively.

In standard conditions, the redox potentials of Sn measured against a standard hydrogen electrode at $25^{\circ} \mathrm{C}$ and ion activity equal to unity are shown in Table $2-1$. This equilibrium reaction needs to be overcome to allow the electrodeposition process to occur. To achieve that, an external power source is connected to the system and additional voltage is applied, thus increasing the negativity of the cathode to facilitate a reduction reaction and increasing the positivity of the anode to facilitate an oxidation reaction. The difference between the applied potential and the potential of the reversible reaction is called the overpotential or overvoltage, as given by the equation below:

Equation 2.12

$$
E_{o v}=E_{a p}-E_{0}
$$

where $E_{o v}$ is the overpotential and $E_{a p}$ is the applied potential. 
Table 2-1 - Standard reduction-oxidation potentials for Sn [30].

\begin{tabular}{|c|c|}
\hline Reaction & Potential (V) \\
\hline $\boldsymbol{S n} \leftrightarrow \boldsymbol{S n}^{2+}+\mathbf{2} \boldsymbol{e}^{-}$ & -0.136 \\
\hline $\boldsymbol{S n}^{2+} \leftrightarrow \boldsymbol{S n}^{4+}+\mathbf{2} \boldsymbol{e}^{-}$ & +0.15 \\
\hline
\end{tabular}




\subsubsection{Efficiency}

The reduction of ions in metal deposition is not the only reaction that takes place during electrodeposition. The reduction of surface oxides, deposition of other metal ions (often a desired effect known as co-deposition), reduction of metal ions and hydrogen evolution are a few examples of other reactions that may occur, which reduce the efficiency of deposition. The current efficiency is defined as the ratio of the current density of the desired reaction (deposition) and the sum of the current density of every other reaction that is occurring. In practice, current efficiency is calculated by the ratio of mass gained by the sample and the theoretical amount that should have been deposited, as calculated by Faraday’s law (Equation 2.9).

During the electrodeposition of Sn, the co-deposition of other metals should not be a concern, as long as the electrolyte is free of impurities. The reduction of metal ions might occur as shown in Equation 2.13 below, but most of $\mathrm{Sn}^{+4}$ ions react with oxygen or hydroxides to form insoluble salts which precipitate, thus changing the electrolyte color from transparent to white. The contribution of this reaction to the total current is in most cases less than $3 \%$ [31].

Equation 2.13

$$
S n^{+4}+2 e^{-} \rightarrow S n^{+2}
$$

Hydrogen evolution has implications that goes beyond the consumption of current. Most of the hydrogen evolved will combine into its gaseous form, which can interfere with deposition adherence by forming bubbles on the surface of the substrate or forming porous deposits.

The deposition process can be conducted by controlling the potential or current. Potential controlled deposition is undertaken to analyze the kinetics of the process. This method would allow the current to vary accordingly, thus reducing the extent of control over the amount deposited. For industrial applications, galvanostatic deposition is usually used due to the increased accuracy of 
the deposition rate. A potential-current density curve, known as a voltammogram, is used to verify the optimum current for deposition.

\subsubsection{Electrodeposition of Sn}

Sn is an amphoteric metal, that is, its ions can react in both acidic and alkaline conditions. Hence, either alkaline (basic) or acidic electrolytes can be used for the electrodeposition of Sn.

\subsubsection{Alkaline Electrolytes}

In alkaline electrolytes, $\mathrm{Sn}$ has a valence of $4+$, rather than $2+$ as is primarily found in acidic electrolytes. This means that alkaline electrolytes require approximately twice as much current to deposit the same mass of Sn at the cathode. This form of deposition is done at a temperature range of $60^{\circ} \mathrm{C}$ to $90^{\circ} \mathrm{C}$, so electrolyte evaporation is a concern.

Alkaline electrolytes are often prepared by simply using metallic stannates and hydroxide. The problem with the use of these electrolytes for deposition is predominantly improper anode control. The wrong conditions could lead to excessive decomposition of hydroxyl ions, thus forming oxygen, and the dissolving of $\mathrm{Sn}$ in the electrolyte as stannite ions (2+) rather than stannate ions (4+), which would lead to porous non-adherent plating.

The deposition efficiency of alkaline-based Sn is limited by two factors: the solubility of the stannates in hydroxide solutions and the cathode efficiency. In most cases, solubility can be increased by increasing the temperature, with sodium stannate being the exception, but cathode efficiency decreases with an increase in temperature. Both factors leads to limitations in the deposition rate of $\mathrm{Sn}[1]$. 


\subsubsection{Acidic electrolytes}

Acidic electrolytes are usually much more complex than alkaline electrolytes because they require additives to improve deposition. Stannous salts used in acidic electrolytes are dissolved in a solution of water and acid, but by itself, these electrolytes will result in deposition with poor adherence and uniformity. Therefore, grain-refining agents, such as gelatin or peptone, are added to acidic electrolytes to improve deposition, but they are usually insoluble in water, and so a wetting agent must also be added. Brighteners can be included as well to improve the resultant surface finish of the deposition. The use of stabilizers will prevent the formation and precipitation of Sn(II) and Sn(IV) salts, increasing the amount of ions available for deposition, which would otherwise be consumed by forming those salts. Only stannous ions $\left(\mathrm{Sn}^{2+}\right)$ are deposited on the surface of the cathode in acidic electrolytes, even though these electrolytes can withstand a large quantity of stannate ions $\left(\mathrm{Sn}^{4+}\right)$ without compromising the efficiency of the system. However, the oxidation of stannous ions to stannate ions must be avoided as this consumes the desired ions. To address that, a large anode is utilized to provide a steady supply of stannous ions, anti-oxidants are added to the solution, the bath temperature is kept low (around room temperature) and special care must be taken in filtration and agitation to limit the amount of oxygen.

Compared to alkaline electrodeposition, the acidic electrodeposition of Sn necessitates half of the current, since it deposits $\mathrm{Sn}^{2+}$ rather than $\mathrm{Sn}^{4+}$, and thus it is a more efficient process. The equipment utilized must be resistant to acidic environments. If bath agitation is used, a filtration system must also be used to remove precipitates that form during the process [1].

\subsection{Modelling}

As mentioned earlier, solutions for the diffusion equation can be obtained with relative ease when the diffusion coefficient is taken as independent. One such solution with particular interest 
to this work is for the case of a finite body with a given initial thickness $h$ that diffuses into a semiinfinite body. The boundary conditions can be set as:

$$
\left.\begin{array}{c}
C=C_{0}, \quad 0<x<h \\
C=0, \quad x>h
\end{array}\right\}, t=0
$$

The differentiation and application of this boundary condition to the diffusion equation can be found elsewhere [22], and the resulting equation is given below with its graphic representation in Figure 2-7.

Equation 2.14

$$
C=\frac{1}{2} C_{0}\left\{\operatorname{erf} \frac{h-x}{2 \sqrt{D t}}+\operatorname{erf} \frac{h+x}{2 \sqrt{D t}}\right\}
$$

where erf is the error function. An interesting property of this solution is that, due to symmetry, the same expression can be applied to the situation where a finite body is trapped between two semi-infinite bodies by setting the origin of the $\mathrm{x}$-axis in the center of the finite diffusing body. 


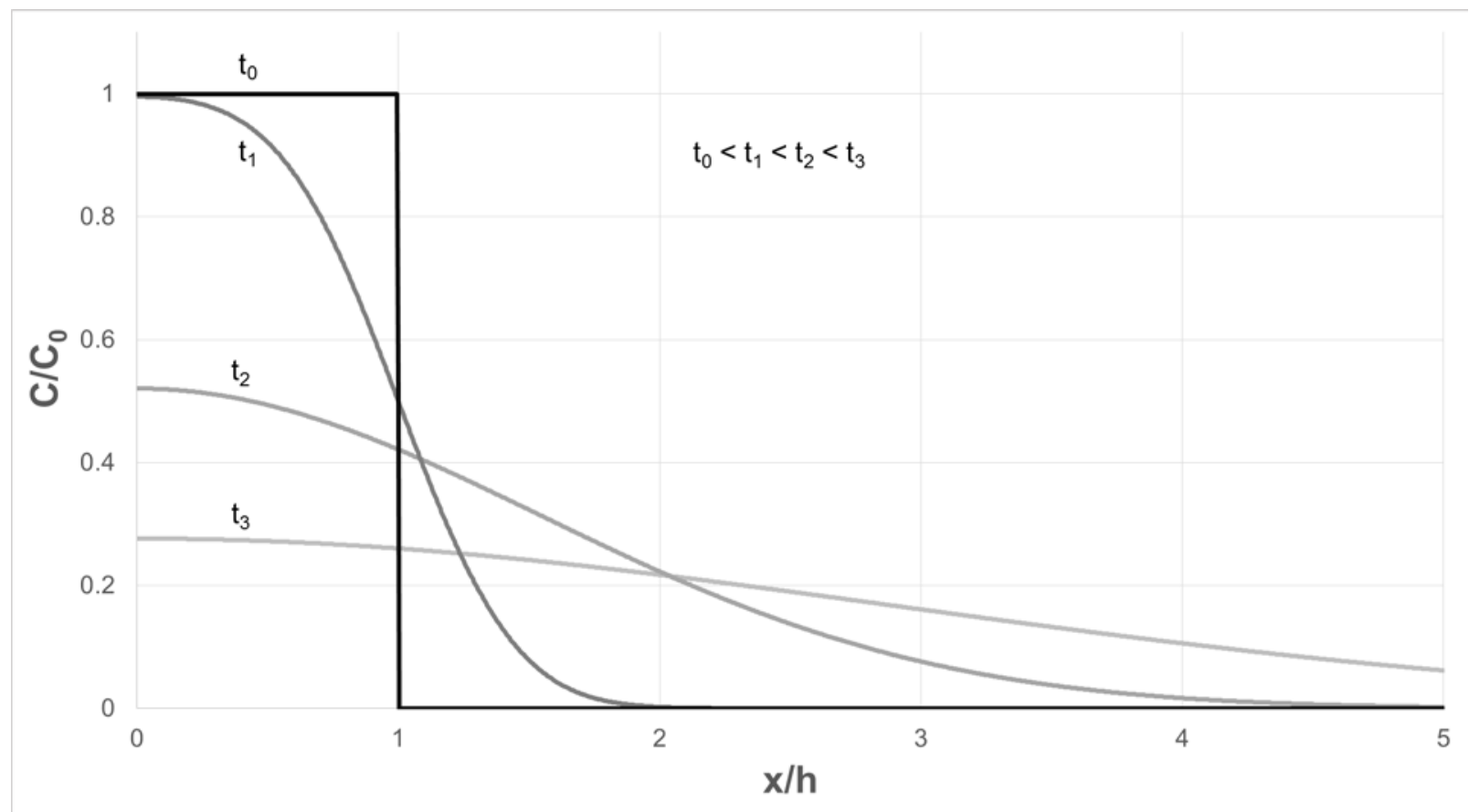

Figure 2-7 - Plot of Equation 2.14 at different points in time. By reflecting around the vertical axis the same equation describes the case of finite body between two semi-infinite bodies. 
Another important analytical solution pertains to the case of diffusion through an interphase, where each side of the boundary is limited by its own saturation concentration. Consider the case where one of the boundary sides has an initial concentration, $\mathrm{C}_{0}$, of 1 , diffusion coefficient, $\mathrm{D}_{1}$, and saturation concentration, which is the lower limit of concentration, s1. The other side of the interphase has an initial concentration of zero, diffusion coefficient, $\mathrm{D}_{2}$, and saturation concentration, which is the upper limit of concentration, s2. In this simple case, the saturation concentrations simply limit the rate of diffusion, and no phase transformation is considered.

At the boundary, $\mathrm{x}=0$, the net flux must be equal on both sides, therefore:

Equation 2.15

$$
D_{1} \frac{\partial C_{1}}{\partial x}=D_{2} \frac{\partial C_{2}}{\partial x} \quad, \quad x=0
$$

By combining the solution of the semi-infinite medium with the expressed boundary condition, the following equation is obtained:

Equation 2.16

$$
\begin{gathered}
C_{1}=C_{0}\left\{1-\frac{\frac{s_{2}}{s_{1}} \sqrt{D_{2}}}{\frac{s_{2}}{s_{1}} \sqrt{D_{2}}+\sqrt{D_{1}}}\left[1+\operatorname{erf}\left(\frac{x}{2 \sqrt{D_{1} t}}\right)\right]\right\} \\
C_{2}=C_{0}\left\{\frac{\frac{s_{2}}{s_{1}} \sqrt{D_{1}}}{\frac{s_{2}}{s_{1}} \sqrt{D_{2}}+\sqrt{D_{1}}}\left[1-\operatorname{erf}\left(\frac{x}{2 \sqrt{D_{1} t}}\right)\right]\right\}
\end{gathered}
$$

It is important to note the limitation of this solution at imposing saturation concentrations. At the interface, $x=0$, Equation 2.16 assumes the following form: 
Equation 2.17

$$
\begin{gathered}
C_{1}=C_{0}\left(1-\frac{\frac{s_{2}}{s_{1}} \sqrt{D_{2}}}{\frac{s_{2}}{s_{1}} \sqrt{D_{2}}+\sqrt{D_{1}}}\right) \\
C_{2}=\frac{C_{0} \frac{s_{2}}{s_{1}} \sqrt{D_{1}}}{\frac{s_{2}}{s_{1}} \sqrt{D_{2}}+\sqrt{D_{1}}}
\end{gathered}
$$

The values of saturation are always in a fraction form; therefore, the actual saturation in the solution will be seen as a function of a fraction $s_{2} / s_{1}$ and the diffusion coefficients $\mathrm{D}_{1}$ and $\mathrm{D}_{2}$, rather than the actual saturation concentrations s1 and s2 individually. Figure 2-8 shows the above solution for $\mathrm{D}_{2}=4 * \mathrm{D}_{1}, \mathrm{C}_{0}=1, \sqrt{D_{1} t}=1$ and $s_{2} / s_{1}=0.5$. Even though the plot shows that saturation takes place at $\mathrm{s}_{1}=0.8$ and $\mathrm{s}_{2}=0.4$, the same curve will be obtained for $\mathrm{s}_{1}=0.4$ and $\mathrm{s}_{2}=$ 0.2 or any other combination of $s_{1}$ and $s_{2}$ in which the fraction equals to 0.5 .

The diffusion process in this study takes place at relatively high temperatures, above the melting point of Sn. Unlike the analytical solution shown above, the solid/liquid interface will not be stationary in this case, due to phase transformation. Moving boundary problems are referred to as Stefan problems which originate from the work of Josef Stefan on the melting of polar ice caps [32]. Solutions to these problems usually rely on an equation known as the Stefan condition:

Equation 2.18

$$
\begin{gathered}
T_{1}=T_{2}=0{ }^{\circ} \mathrm{C} \\
k_{2} \frac{\partial T_{2}}{\partial x}-k_{1} \frac{\partial T_{1}}{\partial x}=L \rho \frac{d S}{d t}
\end{gathered}
$$




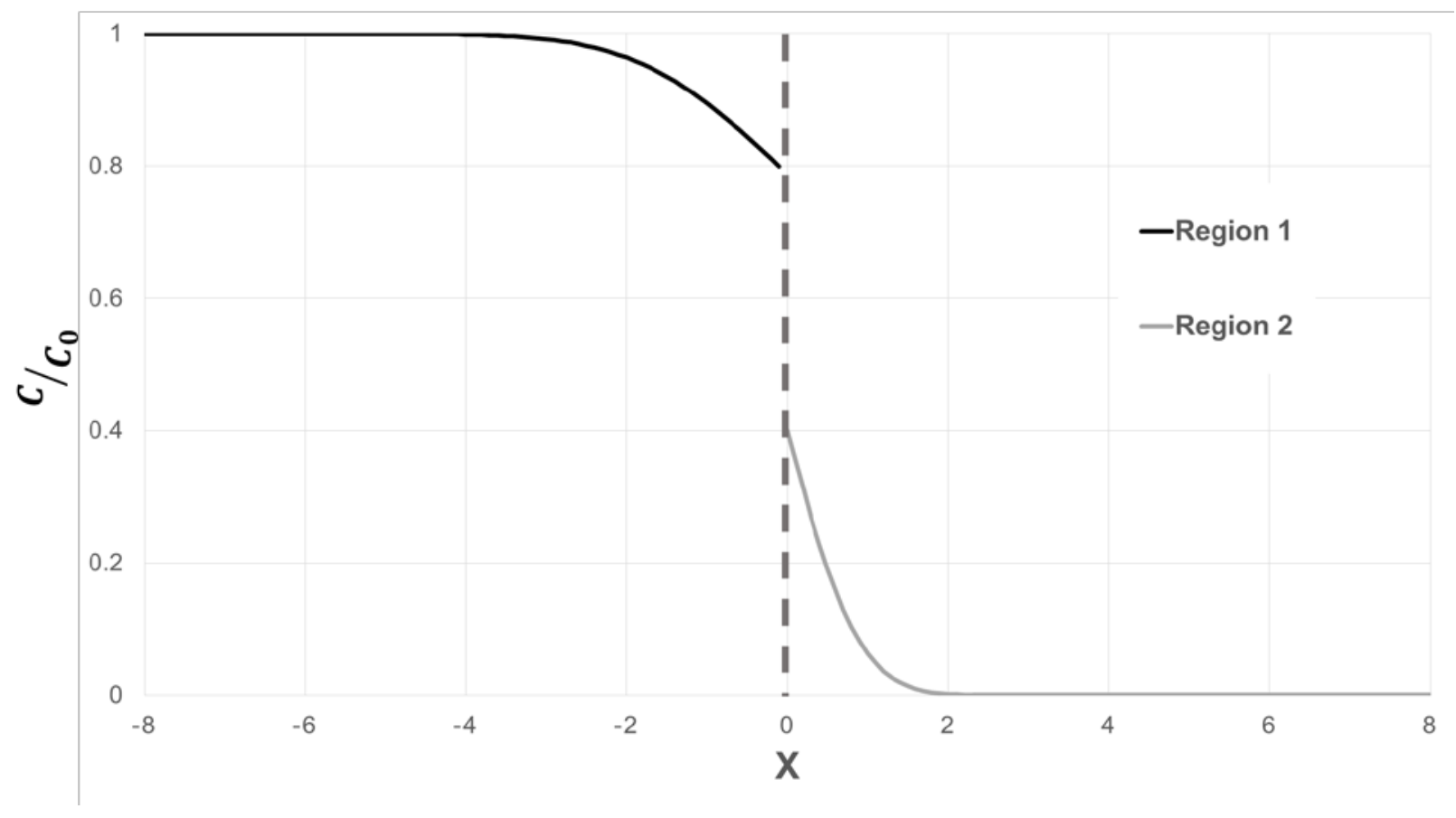

Figure 2-8 - Analytical solution for diffusion on a composite medium. 
Stefan [32] investigated phase changes in water and focused on temperature as the parameter of interest, since that was the cause of phase transformation rather than solute diffusion. In Equation 2.18, subscripts 1 and 2 refer to the liquid and solid phases, respectively, $k$ is the heat conduction coefficient, $T$ is the temperature, $L$ is latent heat and $S$ is the position of the solid/liquid interface.

It is not unusual that heat flow is referenced in diffusion analyses, as both phenomena basically share the same behaviour and can be expressed by using the same equations, given that the appropriate notation changes are made. Equation 2.18 shows that the rate at which an infinitesimal region melts $\left(L \rho \frac{d S}{d t}\right)$ is given by the heat lost by the liquid phase $\left(k_{1} \frac{\partial T_{1}}{\partial x}\right)$ minus the heat gained by the solid phase $\left(k_{2} \frac{\partial T_{2}}{\partial x}\right)$. Translating this same equation into one for a diffusion problem, the concentration needs to change from liquidus to solidus for diffusion induced solidification to occur. The rate at which an infinitesimal region solidifies $\left(\left(C_{12}-C_{21}\right) \frac{d s}{d t}\right)$ is then equal to the amount of solute it gains from the liquid phase $\left(D_{1} \frac{\partial C_{1}}{\partial x}\right)$ minus the amount it loses to the solid phase $\left(D_{2} \frac{\partial C_{2}}{\partial x}\right)$, thus placing them together gives:

Equation 2.19

$$
D_{2} \frac{\partial C_{2}}{\partial x}-D_{1} \frac{\partial C_{1}}{\partial x}=\left(C_{12}-C_{21}\right) \frac{d S}{d t}
$$

where $C_{12}$ and $C_{21}$ are the liquidus and solidus compositions, respectively. The same equation can express diffusion induced melting by reversing the direction of solute diffusion, having concentration change from solidus to liquidus. 
Crank [33] classified the main approaches for solving moving boundary problems into three categories: front tracking, front fixing and fixed domain.

In the front tracking method, the position of the moving boundary is calculated for each point in time. The main problem that arises from this approach is that the movement of the boundary will seldom coincide with the actual grid points of the simulation. Many methods can be used to account for the boundary position. Some examples include that used by Crank and Gupta [34] who utilized unequal space intervals near the moving boundary with a Lagrangian interpolation formula; Murray and Landis [35] who used a fixed boundary as the reference and kept the number of intervals between that and the moving boundary constant by changing the interval between each point; and Douglas and Gallie [36] who kept the spacing constant but adapted a variable time step to ensure that the moving boundary would coincide with a grid point.

In terms of the front fixing method, the moving boundary position is fixed by altering the coordinates used in the system. This transformation was first proposed by Landau in 1950 [37]. This method is often referred to as the arbitrary Lagrangian Eulerian method.

In terms of the fixed domain method, the moving boundary problem is not directly addressed, but instead, reformulated to impose a set of equations onto the system which applies over the whole domain at once. This way, the Stefan condition is implicitly solved, that is, the boundary behaviour is a consequence of the solution, rather than its target. This method is usually applied when boundary movement is erratic, and assumes complicated shapes with sharp peaks or in more than one dimension. 


\subsection{Hardness Test}

The hardness of a surface can be determined with an indentation test; however, this conventional test causes considerable deformation on the surface. For a coated material, the indentations would be significantly influenced by the bulk properties. For such cases, a nanoindentation test can be used to obtain information close to the surface in small volume. Unlike the traditional indentation test, the results of nanoindentation testing are not measured by visually examining the size of the impressions left behind, as they are usually only a few microns in dimension. Instead, the depth of the penetration with respect to the load applied is measured and the area of contact is determined according to the known indenter geometry.

Many indenters are available to carry out nanoindentation hardness testing, which usually have either a pyramidal or conical geometry. The Berkovich indenter is of particular interest for small scale indentation studies because its three-sided pyramid geometry can be brought to a point (with a tip radius between 50-100 $\mathrm{nm}$ ) easier than the Vickers indenter, which has a four-sided pyramid geometry [38]. The Berkovich indenter has a face angle of $65.27^{\circ}$, and provides the same projected area-to-depth ratio as the Vickers indenter.

The projected area of contact given by a Berkovich indenter is provided by the following formula:

Equation 2.20

$$
A=3 \sqrt{3} h_{c}^{2} \tan ^{2} \theta=24.494 h_{c}^{2}
$$

where $A$ is the projected area of contact, $\theta=65.27^{\circ}$ is the face angle and $h_{c}^{2}$ is the depth of penetration by the indenter. Hardness is given by the mean contact pressure, which can be calculated by the ratio of applied load and projected area of contact. 
Nanoindentation instruments must be used in locations that have no acoustic noise, vibrations or temperature variations due to the precision required for measuring nanometer scale movements and micronewton forces. To improve this aspect, the instrument may be installed inside a protective housing located on top of a vibration dampening base. After the sample is mounted onto the nanoindentation instrument, the measurement head is moved vertically until its working distance is reached, which is usually $100 \mu \mathrm{m}$ above the surface of the specimen. After working distance is reached, the indenter is able to move with high precision, which is vital for testing accuracy. The indenter is then slowly moved until contact is made with the specimen surface. This is the reference point used for the following displacement readings, and therefore, the contact force must be minimal to avoid any premature deformation. The initial contact force is usually about $5 \mu \mathrm{N}$ [38].

\subsection{Objective of Current Research}

Diffusion is an interesting area of study as it contains problems that range from simple, where with a series of assumptions a solution can be obtained through manual calculation; to complex, where numerical models are the best, and often the only, option to reach an approximation of the solution. This creates the situation where a specific simplification, that is, an independent diffusion coefficient, is often assumed regardless of its impact on the system behaviour. The main objective of this research is to therefore elucidate the many complex physical processes involved in the heat treatment of $\mathrm{Cu}$ and $\mathrm{Sn}$ and, based on such, develop a numerical model which uses concentration dependent diffusion coefficients to predict the resultant composition profile after heat treatment. 


\section{Chapter 3 Numerical Modeling, Results and Discussion}

As is the case with any simulation investigation, the physical process needs to be understood and its intrinsic mechanisms discussed prior to defining and discretizing the equations to be used in a model. Therefore, Chapter 3 begins with the description of the behavior of a Cu-Sn couple as diffusion occurs at high temperatures, followed by the discretization of the equations utilized for the model and a description of the model itself. After the model is built, its behavior is analyzed by comparing the relative errors with those from the analytical solutions of simple cases. Later, the electrodeposition and heat treatment process used to generate the experimental samples are discussed, and these samples are then used to validate the predictions made by the model. However, the heat treatment process behavior and the numerical model do not take into consideration the presence of Sn-rich craters as mentioned in Chapter 2. A separate discussion on the formation of these craters and a solution to remedy this problem are provided at the end of this chapter.

\subsection{High Temperature Heat Treatment Process Behavior of $\mathrm{Cu}-\mathrm{Sn}$}

Consider that, with the objective of forming an $\alpha$ bronze phase, a $\mathrm{Cu}$ and $\mathrm{Sn}$ couple is placed into a furnace at a temperature above $800^{\circ} \mathrm{C}$ in a protective atmosphere to avoid oxidation. This temperature range is selected to prevent multiple phase transformations as the diffusion of the two

metals occurs, but in doing so, the diffusion between liquid and solid phases, and consequently a moving boundary problem, is introduced. Thus, the heat treatment of this metallic can be described in 5 stages.

The first stage of the heat treatment takes place at a temperature range between room temperature and the melting temperature of Sn, which is $232^{\circ} \mathrm{C}$. The low diffusion rates found at 
low temperatures and the high heating rate due to the imposed large temperature gradient result in little to no diffusion during this stage. Consequently, the model can and will disregard this stage.

The second stage occurs between the melting temperature of Sn and the temperature targeted for heat treatment $\left(+800^{\circ} \mathrm{C}\right)$. Since diffusion during the previous stage was negligible, the solid/liquid interface is virtually in the same location as that of solid/solid interface. However, what was solid Sn is now a slightly thicker liquid Sn, due to the reduced density of the liquid. Since the diffusivity in the liquid phase is orders of magnitude greater than in the solid phase, the overall diffusion rate is limited by solid phase diffusion. The large concentration gradient between the liquid (almost pure $\mathrm{Sn}$ ) and solid (almost pure $\mathrm{Cu}$ ) results in the formation of a steep concentration profile in the solid phase, thus producing a shallow diffusion depth. As diffusion takes place, the solid layer adjacent to the interface quickly increases in concentration of Sn until it reaches the solidus line. After this point, further diffusion will lead to melting of this layer. During this stage, phases other than the targeted $\alpha$ bronze phase will form. The formation of these phases will not be considered by the proposed model. However, a discussion about the consequences will be made in the section on Sn-rich craters. As the specimen reaches the defined working temperature, all of the other phases will dissolve and the third stage begins.

The third stage starts as the diffusion couple reaches the target temperature. As described in the second stage, the solid/liquid interface is moving towards the solid side, that is, the liquid phase is increasing due to melting of the solid phase. As diffusion continues, the concentration of Sn in the liquid phase decreases due to two factors: the diffusion of Sn into the bulk solid and, as the solid becomes liquid, the molten $\mathrm{Cu}$ changes the proportion of $\mathrm{Sn}$ in the liquid phase. Conversely, the solid phase increases the concentration of Sn through the first factor alone, which is the diffusion of Sn. A maximum amount of the solid is dissolved, and consequently, the maximum 
volume of liquid present is found when the concentration of the liquid phase reaches the liquidus line.

The fourth stage begins as the liquid phase reaches liquidus composition at the target temperature. No phase will be stable between the solidus and liquidus compositions, a system that contains an intermediate composition (between solidus and liquidus) will do so only as an average, with a combination of saturated solid (in solidus composition) and saturated liquid (in liquidus composition). Solid will no longer dissolve as the liquid phase reaches saturation, since any dissolution would place the liquid phase below liquidus. However, the diffusion of Sn into the solid will continue, causing the liquid phase to become unstable which leads to the precipitation of saturated solid at the solid surface, at the interface. This precipitation from the liquid phase allows its composition to remain at saturation by consuming its volume.

Just like before, the rate of diffusion of Sn into the solid, and subsequently the solidification at a constant temperature of the liquid phase, is limited by solid state diffusion, but this time, under a much lower concentration gradient, which is at a constant value equal to the difference in the liquidus and solidus concentrations. Therefore, this stage takes considerably longer than the second and third stages. Prematurely stopping the heat treatment process would cause the remaining liquid to solidify and transform into a series of eutectics/eutectoids found at the lower temperatures.

The fifth and final stage starts as the liquid phase completely solidifies. At this stage, just a single $\alpha$-phase is present, with the composition of Sn equal to the maximum level (saturated solid) at the surface where previously liquid was present, and decreasing towards the bulk. Stopping the heat treatment process at the beginning of this stage would result in the maximum content of $\mathrm{Sn}$ as the $\alpha$-phase at the surface. As this stage progresses, the concentration of Sn on the surface decreases through solid state diffusion. 
Figure 3-1 shows three diagrams that depict the stages described above. In each diagram, a lighter shade of gray denotes the system at a later moment in time. Everything above the liquidus composition $\left(\mathrm{C}_{\mathrm{L}}\right)$ can be considered liquid and everything below the solidus composition (Cs) can be considered solid. Note that for any position $\mathrm{x}$ in any of the diagrams, the composition is either below $\mathrm{Cs}_{\mathrm{S}}$ or above $\mathrm{C}_{\mathrm{L}}$; on the solid/liquid interface there is always discontinuity in the composition. Due to the high diffusivity in the liquid phase, its composition is shown to be homogeneous at all times.

Figure 3-2 shows how the composition for the solid and liquid phases change during the first three stages. As mentioned, the composition changes during the first stage are negligible compared to those in the second and third stages. Since the liquid phase is considered to have a virtually homogeneous composition, it is easy to visualize how this part of the system changes with time. Contrariwise, the solid phase will not be homogeneous. Each layer parallel to the interface will have a different concentration of Sn for a given time. Therefore, the curve on the left does not illustrate the composition changes of the solid phase as a whole, but rather, for a layer close to the interface. As described above, the fourth stage occurs at the solidus and liquidus lines and the fifth stage has the remaining solid phase shifting towards the left on the phase diagram. Once again, neither the solid nor the liquid phase enters the region between the solidus and liquidus compositions. 
a)

b)

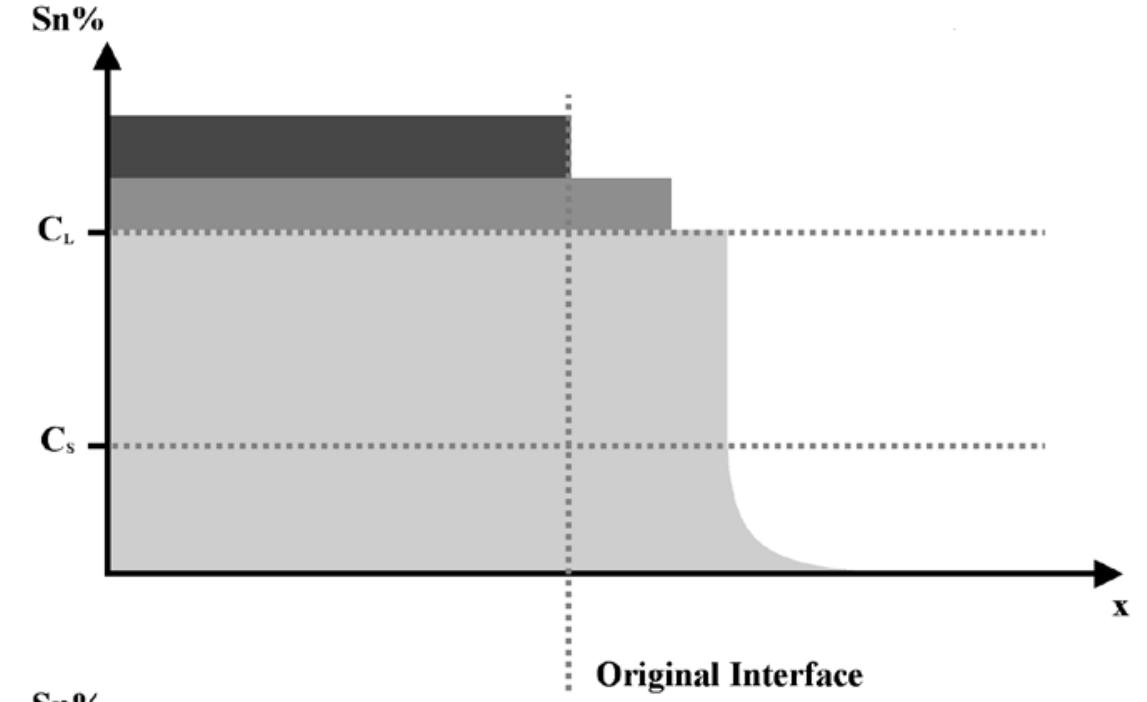

c)
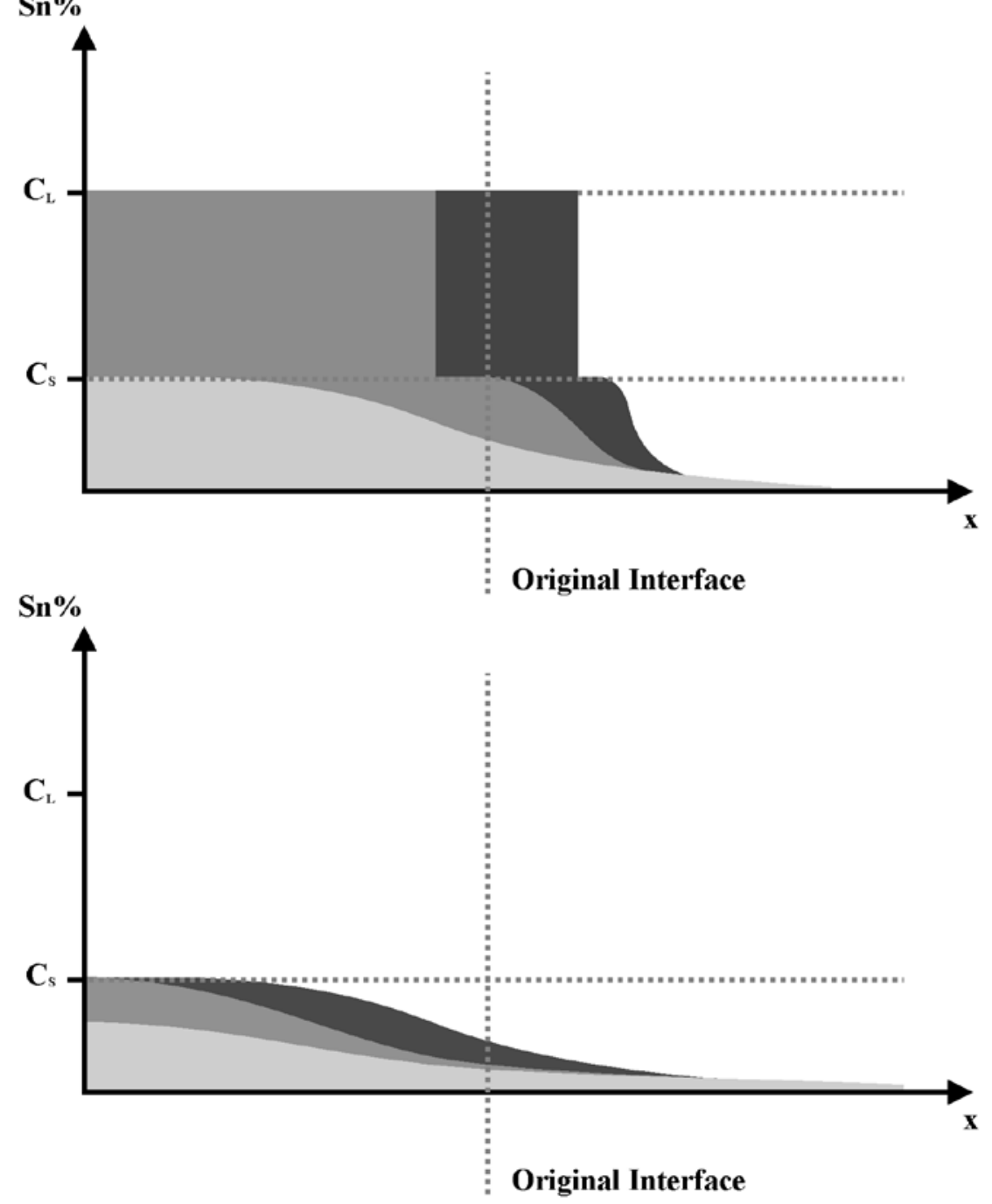

Figure 3-1 - Stages a) I, II and III, b) IV and c) V. Lighter shades of gray represents a later point in time. 


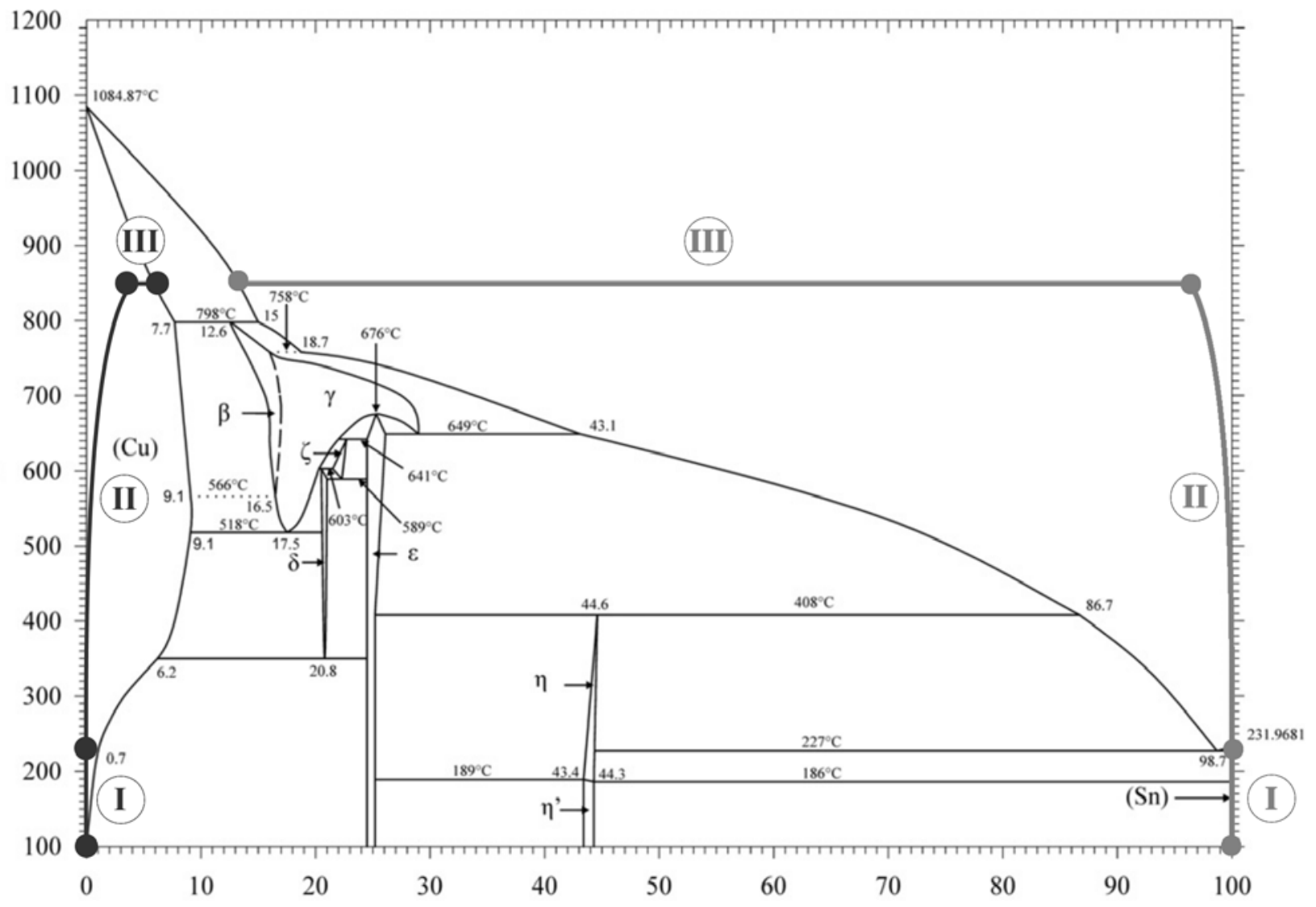

Figure 3-2 - Stages I, II and III for a layer of copper (left) and tin/liquid phase (right). Phase diagram based on [5]. 


\subsection{Development of Numerical Model}

The proposed model was built in one-dimensional Cartesian space, discretized by a finite difference method with a fixed number of nodes but variable spacing around the moving boundary, which was adapted from Patankar's work on heat conduction [39]. For a one-dimensional analysis, the front tracking method is a sufficiently robust technique for calculating the moving boundary position, therefore, it was chosen to avoid increasing the model's computational cost. The composition and diffusion coefficient are dependent variables, while the position, time and temperature are independent variables:

\subsubsection{Discretization}

$$
\begin{gathered}
C=C(x, t) \\
D=D(C, T)=D(x, t, T)
\end{gathered}
$$

During discretization, the simulated body is represented by discrete points where the solution is calculated, as shown in Figure 3-3. Calculation is done at the center node, shown as 1, within a control volume with a size of $\Delta \mathrm{x}$, which accommodates the influence of its neighbors, 0 and 2 .

The general approach to obtaining the solution consists of using the initially known variables in point $x$ at time $t$ to calculate variables at time $t+\Delta t$, and repeating the same procedure for the next point $\mathrm{x}+\Delta \mathrm{x}$. After every point in the simulated body is calculated, time is advanced (i.e. $t=$ $\mathrm{t}+\Delta \mathrm{t}$ ) and the process is repeated. In the following equations, the superscripts indicate present (i)

or future (f) values and subscripts represents the grid point location, e.g., $C_{1}^{i}$ indicates the present composition at grid point 1 .

For convenience, Fick`s second law of diffusion is provided again below.

\section{Equation 3.1}

$$
\frac{\partial C}{\partial t}=\frac{\partial}{\partial x}\left(D \frac{\partial C}{\partial x}\right)
$$




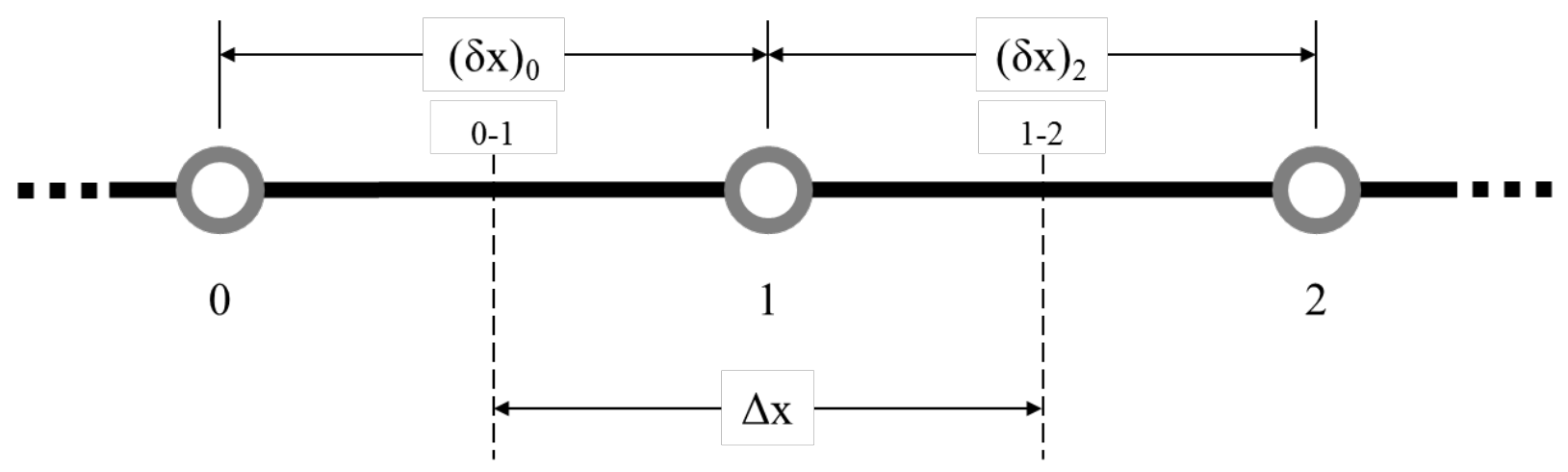

Figure 3-3 - One-dimensional grid. 
Integrating Fick`s second law of diffusion over the control volume for the time interval from $\mathrm{t}$ to $\mathrm{t}+\Delta \mathrm{t}$ :

Equation 3.2

$$
\int_{0-1}^{1-2} \int_{t}^{t+\Delta t} \frac{\partial C}{\partial t} d t d x=\int_{t}^{t+\Delta t} \int_{0-1}^{1-2} \frac{\partial}{\partial x}\left(D \frac{\partial C}{\partial x}\right) d x d t
$$

Assuming that, for any time, the composition is constant throughout the control volume $\Delta \mathrm{x}$, the left side of Equation 3.2 becomes:

Equation 3.3

$$
\int_{0-1}^{1-2} \int_{t}^{t+\Delta t} \frac{\partial C}{\partial t} d t d x=\Delta x\left(C_{1}^{f}-C_{1}^{i}\right)
$$

Moving to the right side of Equation 3.2, the spatial integration can be solved as:

Equation 3.4

$$
\int_{t}^{t+\Delta t} \int_{0-1}^{1-2} \frac{\partial}{\partial x}\left(D \frac{\partial C}{\partial x}\right) d x d t=\int_{t}^{t+\Delta t}\left[\frac{D_{1-2}\left(C_{2}-C_{1}\right)}{(\delta x)_{2}}-\frac{D_{0-1}\left(C_{1}-C_{0}\right)}{(\delta x)_{0}}\right] d t
$$

To integrate Equation 3.4 over time, it is first necessary to define how the composition, and consequently the diffusion coefficient, in each grid point will change with time. Possible assumptions fall under three broad categories: explicit, implicit and fully-implicit schemes.

The explicit scheme assumes that the change from $C^{i}$ to $C^{f}$ as time progresses from $t$ to $t+\Delta t$ occurs stepwise, staying constant and equal to $C^{i}$ until a sudden change to $C^{f}$ at $t=t+\Delta t$, hence:

Equation 3.5

$$
\int_{t}^{t+\Delta t} C d t=C^{i} \Delta t
$$


This way, assuming a constant grid spacing equal to $\Delta \mathrm{x}$, Equation 3.2 becomes:

Equation 3.6

$$
\frac{\Delta x}{\Delta t} C_{1}^{f}=\frac{D_{0-1}}{\Delta x} C_{0}^{i}+\frac{D_{1-2}}{\Delta x} C_{2}^{i}+\left(\frac{\Delta x}{\Delta t}-\frac{D_{0-1}+D_{1-2}}{\Delta x}\right) C_{1}^{i}
$$

Since the composition of a point in the system is influenced by the composition of its neighbors, it is easy to see that an increase in the solute in a neighboring location would always lead to a compositional increase of the central point; a composition decrease would indicate inconsistency of the physical description of the model. To avoid any inconsistencies, the coefficients multiplying the composition must always be positive. Therefore, for the explicit scheme, the following stability criterion must be obeyed to ensure convergence:

\section{Equation 3.7}

$$
\frac{\Delta x}{\Delta t}-\frac{D_{0-1}+D_{1-2}}{\Delta \mathrm{x}}>0 \quad \longrightarrow \quad \Delta t<\frac{(\Delta x)^{2}}{\left(D_{0-1}+D_{1-2}\right)}
$$

The fully-implicit scheme follows a similar logic for composition change with time; it also occurs in a stepwise manner, but the composition assumes its future value during the transition from t to $\mathrm{t}+\Delta \mathrm{t}$, hence:

Equation 3.8

$$
\int_{t}^{t+\Delta t} C d t=C^{f} \Delta t
$$

Thus, Equation 3.2 assumes the following form:

Equation 3.9

$$
\left(\frac{D_{0-1}+D_{1-2}}{\Delta x}+\frac{\Delta x}{\Delta t}\right) C_{1}^{f}=\frac{D_{0-1}}{\Delta \mathrm{x}} C_{0}^{f}+\frac{D_{1-2}}{\Delta \mathrm{x}} C_{2}^{f}+\frac{\Delta x}{\Delta t} C_{1}^{i}
$$


Unlike the explicit scheme, the fully-implicit scheme will always have positive coefficients, and does not require any criteria to be followed to ensure a physically meaningful result. However, the calculation of $C_{1}^{f}$ relies on unknowns, thus requiring a set of equations to be simultaneously solved. Furthermore, a concentration dependent diffusion coefficient would need to be interactively calculated from $C_{1}^{f}$.

Between the extremes of explicit and fully-implicit schemes lies implicit schemes, where the composition changes with time are set to have a certain profile, and its integration usually assumes the following form:

Equation 3.10

$$
\int_{t}^{t+\Delta t} C d t=\left[F C^{f}+(1-F) C^{i}\right] \Delta t
$$

where $\mathrm{F}$ is a weighing factor that can vary between 0 (explicit) and 1 (fully-implicit). Some notable examples are the Crank-Nicolson method [40], which sets $F=0.5$, thus resulting in higher accuracy but still requiring $\Delta \mathrm{t}$ to be sufficiently small to obtain realistic results. There is also the Baliga-Patankar method [41], which sets an exponential profile for composition variance, thus increasing accuracy and eliminating any limits on $\Delta \mathrm{t}$ to be realistic, but at the expense of increased mathematical complexity.

Assuming that the conditions to obtain physically meaningful results are observed, all of the aforementioned schemes provide the same result, albeit at different rates. Therefore, by conforming to the criterion to ensure convergence, the explicit method is more suitable to be used for the present work. 


\subsubsection{Boundary Conditions}

\subsubsection{Free surface}

The free surface boundary condition occurs at the limits of the sample, and effectively denotes the end of the sample and start of the atmosphere it is immersed in. For problems that involve surface treatment, as is the present case, a semi-infinite body is often assumed, that is, a sample that is large enough so that its second free surface does not affect the process analyzed. Therefore, a single free surface, which is the Sn-rich side, will be considered in this study.

No mass flux is found throughout the free surface, which means that any changes in concentration that occur on that point is caused only by one neighbor control volume. Consequently, the concentration of the first point can be calculated by integrating the half control volume, as illustrated in Figure 3-4.

Repeating the process described during discretization but making the appropriate changes due to the absence of one neighboring point and the fact that $\Delta \mathrm{x}^{\prime}=0.5 \Delta \mathrm{x}$, the explicit equation for the free surface boundary condition is:

Equation 3.11

$$
\frac{\Delta x}{2 \Delta t} C_{1}^{f}=\frac{D_{1-2}}{\Delta x} C_{2}^{i}+\left(\frac{\Delta x}{2 \Delta t}-\frac{D_{1-2}}{\Delta x}\right) C_{1}^{i}
$$

Just like with Equation 3.6, a similar stability criterion must be obeyed:

Equation 3.12

$$
\Delta t<\frac{\Delta x^{2}}{2 * D_{1-2}}
$$




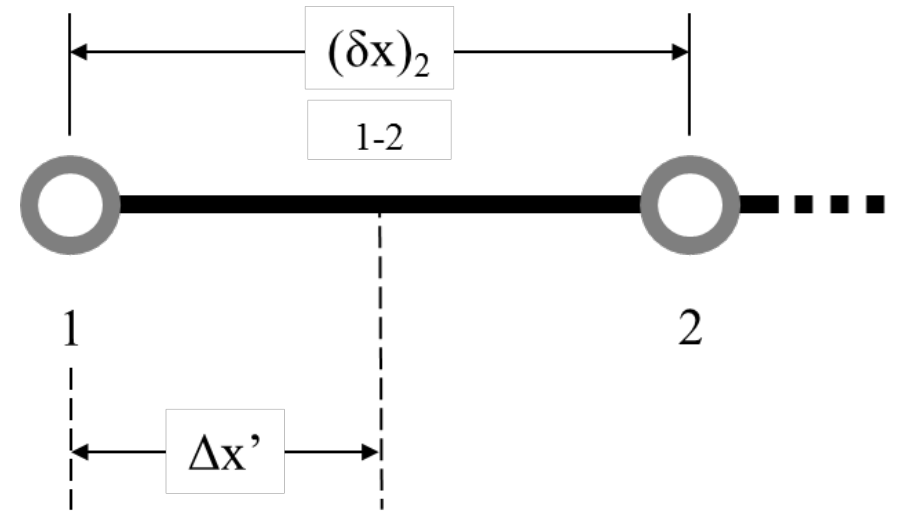

Figure 3-4 - One-dimensional grid showing the half control volume at a free boundary. 


\subsubsection{Moving interface}

The moving interface boundary condition is found any time a gradual phase change occurs. For the diffusion process under analysis, this boundary condition will be mainly applied during the solidification stage due to the diffusion between the saturated liquid and solid. As discussed in Chapter 2, these are known as Stefan problems. For convenience, the Stefan condition for diffusion is provided again below.

Equation 3.13

$$
D_{2} \frac{\partial C_{2}}{\partial x}-D_{1} \frac{\partial C_{1}}{\partial x}=\left(C_{12}-C_{21}\right) \frac{d S}{d t}
$$

The boundary movement rate is controlled by the diffusion that occurs through the boundary. As mentioned before, the liquid phase cannot be stable at a concentration below liquidus, likewise, the solid phase cannot be stable above solidus. This creates a stepwise concentration gradient, where the concentration profile jumps from a high amount of Sn on the liquid phase to a low amount at the solid phase. Thus, a volume of liquid solidifying or a volume of solid melting will have an abrupt transition of composition. In order to do so, a control volume needs to receive, or lose, enough solute (Sn). Therefore, the net flow of solute necessary to transform a control volume with size $\Delta \mathrm{x}$, is:

Equation 3.14

$$
\left(C_{12}-C_{21}\right) \Delta x=\left(D_{2} \frac{\partial C_{21}}{\partial x}-D_{1} \frac{\partial C_{12}}{\partial x}\right) t
$$

So far, the described model calculates the diffusion process by assessing the solute concentration change in each grid node, increasing time by dt and repeating the process. Simply calculating the interface position with this logic would, more often than not, lead to an interface located somewhere between two grid points. Generally, with the front tracking method, this 
problem can be solved by either altering the simulated grid or the simulation time step. The method chosen for this work is a combination of both approaches. As outlined in the beginning of this chapter, the case in point will display a moving interface in two distinct situations: saturated solid and non-saturated liquid, which occurs during the second and third stages, and saturated solid and liquid, which take place during the fourth stage.

When the first layer of the solid reaches saturation, its composition is kept constant until enough solute have diffused for its phase change. Equation 3.6 can be rewritten to determine the time necessary for a control volume $\Delta \mathrm{x}$ to change its solute concentration from saturated solid to saturated liquid, as seen below.

Equation 3.15

$$
t=\frac{\Delta x^{2}\left(C_{L}-C_{S}\right)}{\left(D_{0-1}\left(C_{0}^{i}-C_{S}\right)+D_{1-2}\left(C_{2}^{i}-C_{S}\right)\right)}
$$

As mentioned, the situation described occurs throughout the second and third stages. During the second stage, the temperature changes with time, and with that, so does the diffusion coefficient and saturation concentrations. All of these are important factors in Equation 3.15. Therefore, during this stage, the time step is limited by a fraction of the time required for the boundary to move a distance of $\Delta \mathrm{x}$ and time is continuously recalculated with new values of temperature, diffusion coefficient and saturation concentrations. The solid control volume at the solid/liquid interface is saturated, therefore, further diffusion of Sn should cause a fraction of this layer of solid to melt. This factor is also important since the dissolution of this previously solid fraction results in a decrease of the liquid concentration, thus reducing the concentration gradient across the interface and, consequently, the diffusion rate. To account for this, a virtual movement of the boundary is created by adjusting the distance between the liquid and solid control volumes at the 
interface. Assuming that the first layer of solid is identified as Node 1 and the liquid is on its left, this distance is represented by $(\delta \mathrm{x})_{0}$ in Figure 3-3. Therefore, Equation 3.6 is rewritten as:

Equation 3.16

$$
\frac{\Delta x}{\Delta t} C_{1}^{f}=\frac{D_{0-1}}{(\delta x)_{0}} C_{0}^{i}+\frac{D_{1-2}}{(\delta x)_{2}} C_{2}^{i}+\left(\frac{\Delta x}{\Delta t}-\frac{D_{0-1}}{(\delta x)_{0}}-\frac{D_{1-2}}{(\delta x)_{2}}\right) C_{1}^{i}
$$

where:

$$
\Delta x=\frac{(\delta \mathrm{x})_{0}}{2}+\frac{(\delta \mathrm{x})_{2}}{2}
$$

While altering the time step affects the system as a whole, virtual movement of the boundary is only applied on the two adjacent nodes located at the interface.

During the third stage, the temperature is taken as a constant, which limits the impact of the large time steps. However, the partial movement of the boundary is still a concern, thus it is applied in this stage as well.

In terms of the second situation, the fourth stage, both liquid and solid are in their saturated conditions. The liquid phase can no longer accommodate any new solid dissolution. This creates an interesting circumstance where the previously determined equations would lead to physically inconsistent behavior. That is, the concentration gradient would cause solute from the liquid phase to continue to diffuse into the solid, which causes the solid phase to dissolve, which in turn would cause the composition of the liquid to be below the liquidus concentration. Therefore, the rate of solidification at a constant temperature is controlled by solid state diffusion, meaning that regardless how fast diffusion takes place in the liquid phase, it will be limited by the rate of diffusion into the solid. The solid control volume at the interface must first diffuse solute towards the bulk, bringing its concentration below solid saturation, to then receive solute from the liquid 
phase, thus obeying its saturation limitation. Therefore, the time necessary for a liquid control volume $\Delta \mathrm{x}$ to solidify can be inferred by the time necessary for the solid control volume at the interface to diffuse the same amount of solute mass toward its bulk. Consequently, the Stefan condition for this situation can be rewritten as:

Equation 3.17

$$
-D_{1} \frac{\partial C_{S}}{\partial x}=\left(C_{L}-C_{S}\right) \frac{d S}{d t}
$$

Thus, the time necessary for the solidification of $\Delta \mathrm{x}$ can be calculated by assuming a free boundary between the solid and liquid phases. In this way, similarly to Equation 3.15, the exact amount of solute that have diffused out of the solid control volume, towards the solid bulk, can be determined.

Equation 3.18

$$
t=\frac{\Delta x^{2}\left(C_{L}-C_{S}\right)}{D_{1-2}\left(C_{S}-C_{2}^{i}\right)}
$$

Unlike what was observed during the previous stages, the magnitude of the time step during solidification has little influence on the final behavior of the boundary, provided that it is below the limit for convergence. At the interface, both solid and liquid are, and remain at, saturation; and temperature is kept constant. Therefore, none of the previous parameters undergo any changes over time. For this reason, during this stage, the simulation is simplified: $\Delta \mathrm{x}$ is fixed and $\Delta \mathrm{t}$ is calculated by using Equation 3.18 as long as convergence is criterion obeyed.

\subsubsection{Variable Diffusion Coefficient}

As mentioned before, the diffusion coefficient often displays a meaningful dependency with concentration. Fortunately, the discretization of the problem presented allows for this dependence, thus permitting the diffusion coefficient to be calculated at any point in space and time. However, 
the value of the diffusion coefficient utilized in the discretized equations is not the same as that on the point where the composition is calculated, rather, it is the one assumed at the borders of the defined control volume ( $\left.\mathrm{D}_{0-1}, \mathrm{D}_{1-2}\right)$. Intuitively, one can assume that linear interpolation would result in a correct approximation at said borders, but that is not the case.

Fick's first law of diffusion, as shown below, can be used to determine the flux of mass through the border of the control volume between Nodes 0 and 1.

Equation 3.19

$$
J=-D_{0-1} \frac{\left(C_{1}-C_{0}\right)}{\Delta x}=-\frac{\left(C_{1}-C_{0}\right)}{\Delta x / D_{0-1}}
$$

Since the composition is set to be constant inside each individual control volume, the diffusion coefficient inside each control volume will be considered to be constant as well $(\Delta x / D=$ cte). Referring back to Figure 3-3, it can be seen that from the node 0 to the node 1 , only half of each volume of controls are contributing to the flux $\left(\Delta x / 2 D_{0}+\Delta x / 2 D_{1}\right)$. Assuming a constant size, $\Delta \mathrm{x}$, for each control volume, Equation 3.19 can be rewritten as:

Equation 3.20

$$
J=-\frac{\left(C_{1}-C_{0}\right)}{\frac{\Delta x}{2} \frac{1}{D_{0}}+\frac{\Delta x}{2} \frac{1}{D_{1}}}
$$

The correct value for $\mathrm{D}_{0-1}$ would result in the same flux obtained from both Equation 3.19 and Equation 3.20, and by equating those equations, the correct expression is found:

Equation 3.21

$$
D_{0-1}=\frac{2 D_{0} D_{1}}{D_{0}+D_{1}}
$$


Therefore, a harmonic mean is the correct expression for calculating the diffusion coefficient at the border of each control volume rather than an arithmetic mean, as would be given by using linear interpolation.

\subsection{Analysis of Numerical Model}

\subsubsection{Comparison Against Finite/Semi-infinite Solid Diffusion}

Comparing the model described above with an analytical solution for the diffusion of a finite/semi-infinite couple with a constant diffusion coefficient, the accuracy of the discretization process for the basic equations and the free boundary condition can be ascertained.

\subsubsection{Effect of Time Step - $\Delta t$}

During discretization, it was determined that the current value of concentration transforms into the future value in a stepwise manner, in which the former is retained throughout the passing of $\Delta \mathrm{t}$, which creates an upper limit ( $\left.\Delta \mathrm{t}_{\max }\right)$ above which the simulation cannot provide realistic results. Therefore, the analysis will always be maintained below the upper limit of the time step.

The following results were obtained by comparing an analytical solution and the simulation results with the following parameters: free surface on the left; finite solid initial concentration $\mathrm{C}_{0}$ $=1$; finite solid thickness $\mathrm{h}=5$; semi-infinite solid initial concentration $\mathrm{C}_{\text {inf }}=0$; constant diffusion coefficient $\mathrm{D}=0.1$; diffusion time $\mathrm{t}=500$; simulation time step and spatial step as indicated. It is important to note that for these simulations, the boundary condition of the behavior of the material is not implemented.

Analytical solutions are capable of calculating the concentration at any point in the domain; however, for comparison purposes, discrete points spaced apart by $\Delta \mathrm{x}$ were calculated, as they should be equivalent to those obtained from the simulation. 
Figure 3-5 shows the error distribution along the simulated length for different of $\Delta t$ and $\Delta \mathrm{x}$. It is evident that when $\Delta \mathrm{t}$ is set to the convergence limit, error is maximized. The shape of the curve shows the stepwise approximation, alternating between peaks of maximum error value and valleys where errors are virtually null. As $\Delta$ t decreases, peaks and valleys converge to a line where errors become a smooth curve, with the maximum error roughly equal to half of that observed with $\Delta t_{\text {max. }}$ This reduction is not coincidental, and in fact, is expected from the discretization process applied, because as $\Delta t$ decreases, error is distributed among every point of the simulated solution. Figure 3-6 shows the superposition of the analytical solution and the curve approximated by the model with a large $\Delta \mathrm{t}$ and a small one. Since mass is set to be conserved, a time step reproduction of a concentration curve cannot have all of the steps intercepting the curve, otherwise, the total mass would be over or underestimated. This can be observed when the analytical solution is treated like a discrete solution. If the total mass under the analytical composition curve in the initial conditions is calculated by multiplying the concentration of a point by the distance between the points, $\Delta \mathrm{x}$, the mass would be overestimated by $\frac{C * \Delta x}{2}$, as shown on Figure 3-6b. As diffusion time increases, the slope of the concentration curve is decreases, as is the case with the overestimation of mass in the system. To maintain constant mass, the model mainly behaves in two different ways. At $\Delta \mathrm{t}_{\max }$, the calculated concentration alternates between values of low error, close to real solution, and values of large error, forming the stepwise shape of the approximation and guaranteeing that mass is not overestimated, Figure 3-6c. A reduced $\Delta \mathrm{t}$ causes error, previously concentrated on a few points, to be distributed among every point, reducing the overall error to half of its original value and smoothing the stepwise approximation down to a curve, Figure 3-6d. By varying the diffusion time $(\mathrm{t}=0.01 \sim 1000)$, it became evident that error is concentrated around the interface, where the concentration gradient is largest, and reduced as the diffusion time increases, due to the decrease of the slope of the composition curve. 

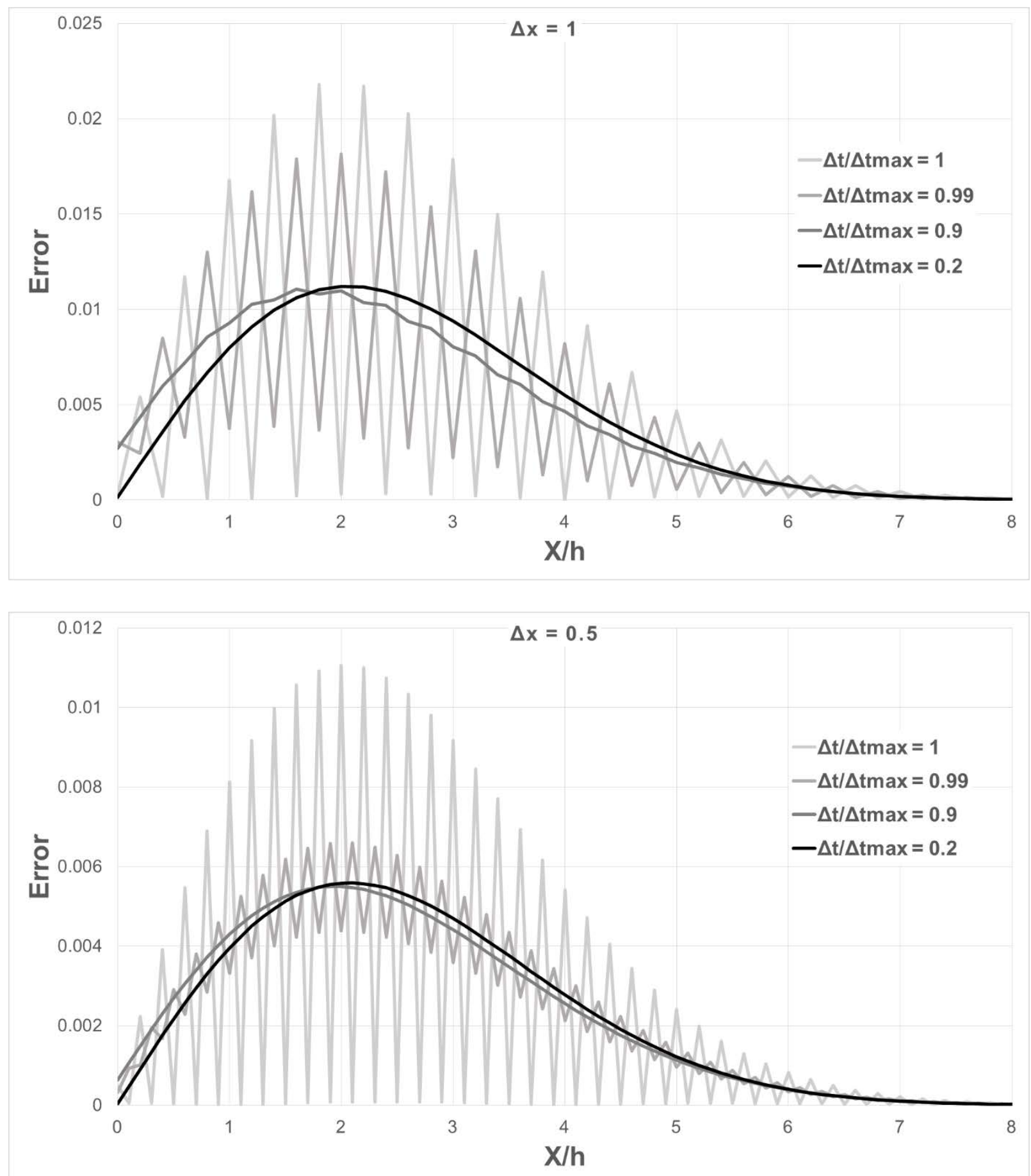

Figure 3-5 - Error behavior at different values of $\Delta t$ and $\Delta x$ (continues on next page). 

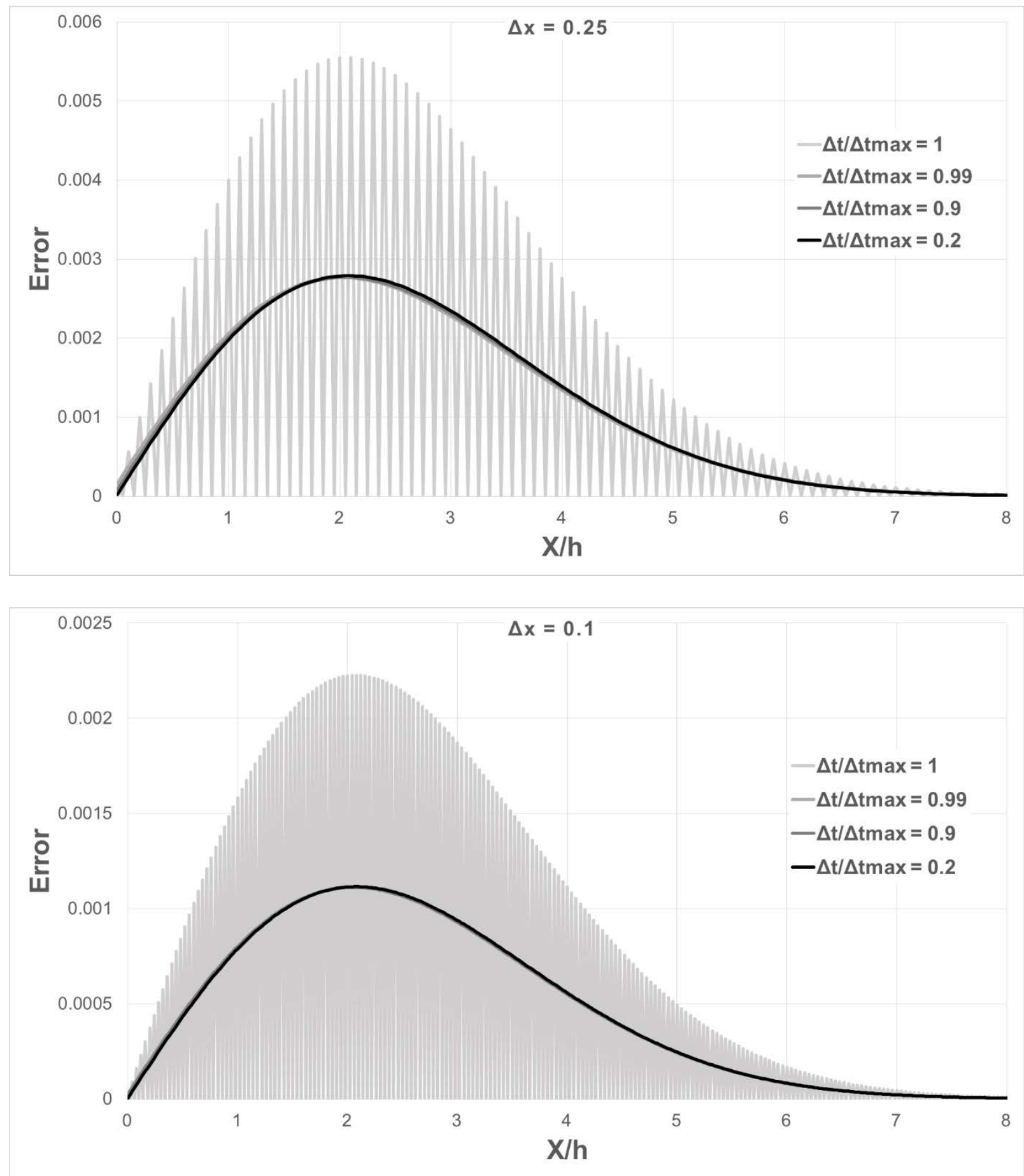

Figure 3.5(continued) - Error behavior at different values of $\Delta t$ and $\Delta x$. 

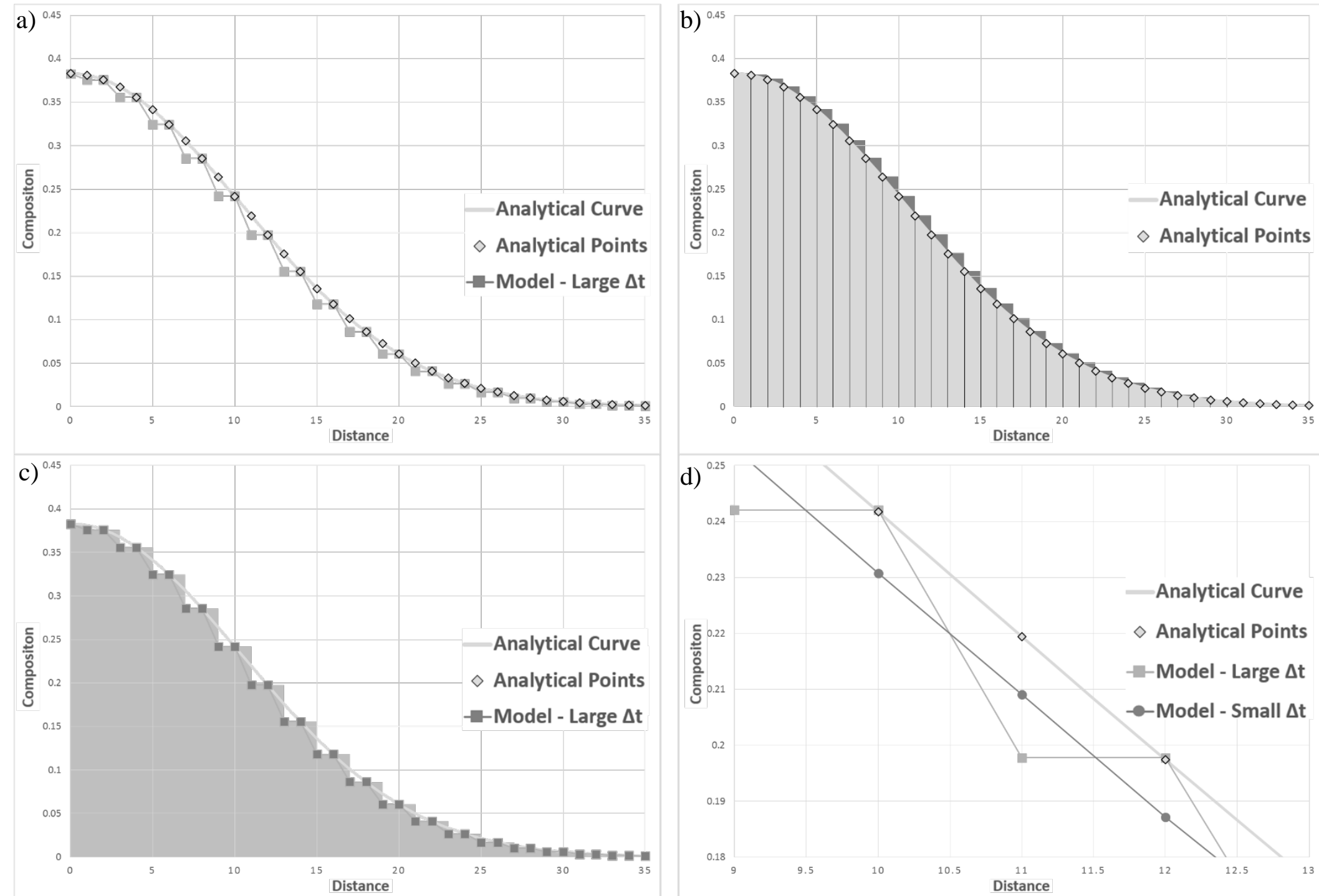

Figure 3-6 - a) Analytical curve superimposed by model approximation with $\Delta t_{\max }$; b) Area under the curve of discrete analytical points, darker region shows where mass is being overestimated. c) Mass calculated from area under model approximation; d) detail showing analytical curve, and curve approximated by the model with large and small $\Delta t$. 
A simulation model incrementally calculates diffusion by utilizing the previous condition in time to calculate the next. This is in contrast with an analytical equation, which only depends on the initial conditions to calculate any point in time. The fact that error is not increased with an increase in the diffusion time indicates that it is indeed caused by the approximation of the curve with discrete points, since no error accumulates. Therefore, the discretization of the governing equations is sound as is the actual implementation of the computation code.

\subsubsection{Effect of Spatial Step $-\Delta x$}

Unlike $\Delta \mathrm{t}$, decreases in $\Delta \mathrm{x}$ will always result in increased accuracy of the model. Each graph in Figure 3-5 corresponds to a different $\Delta \mathrm{x}$ size and even though the overall shape remains the same, the error range is directly proportional to $\Delta \mathrm{x}$. Figure 3-7 shows the maximum error as it changes with $\Delta \mathrm{x}$ for various values of diffusion coefficient, all at the same diffusion time. The value of $\Delta t$ is set to be small enough so as to ensure an average error, as explained before. It can

be seen that a linear relation of the form Error $=A * \frac{\Delta \mathrm{x}}{D}$ exists, where A is a variable associated with the slope of the concentration curve, which in turn, can be correlated to the diffusion time. 


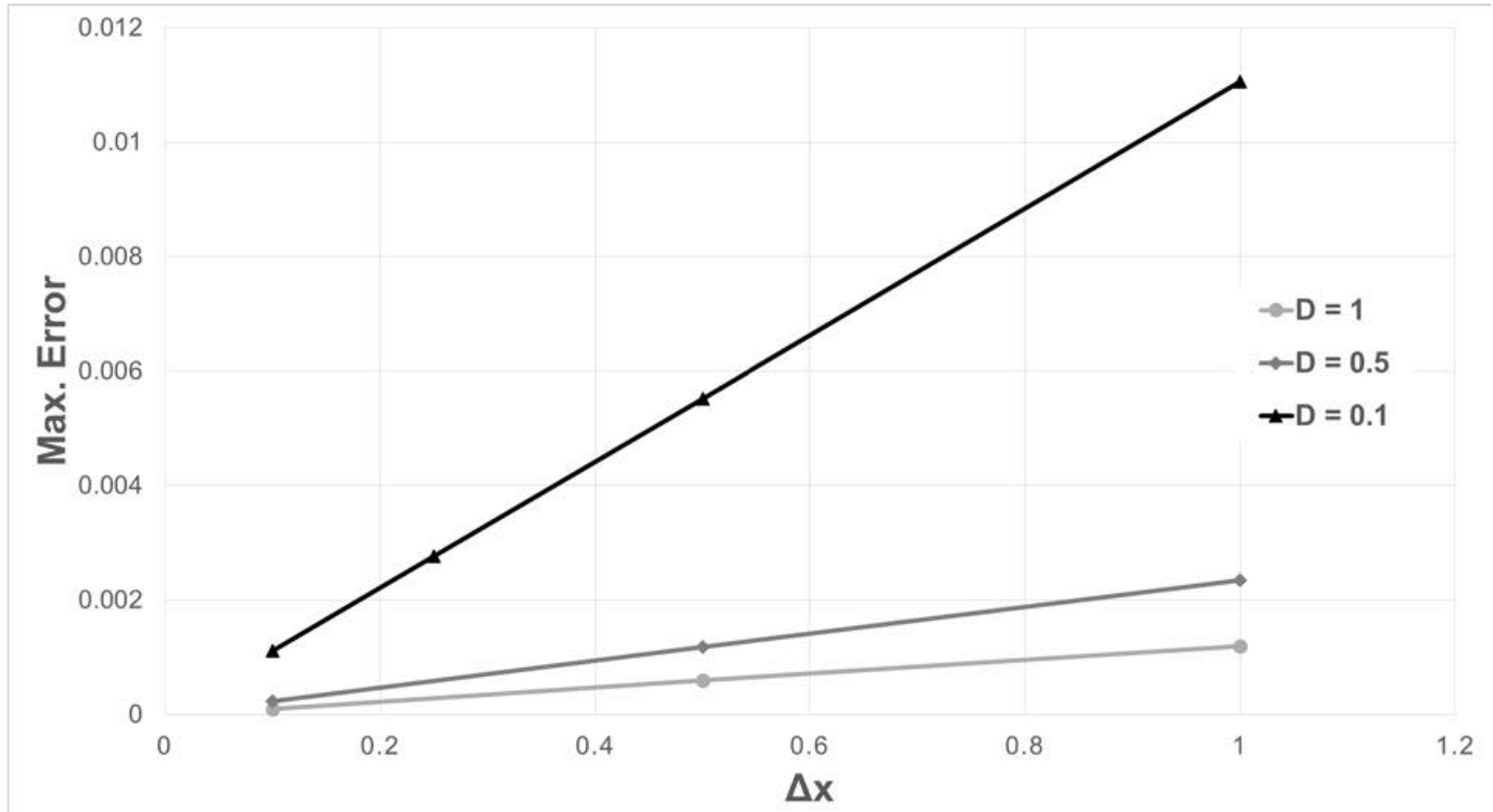

Figure 3-7 - Error propagation with respect to $\Delta x$. 


\subsubsection{Comparison Against Semi-infinite Composite Medium}

A semi-infinite composite medium consists of two regions with different initial concentrations, diffusion coefficients and saturation. For this comparison, the interface of the two regions will be set as the zero of the system, Region 1 will be confined to the negative side ( $\mathrm{x}<$ 0), with diffusion coefficient $\mathrm{D}_{1}$ and saturation $\mathrm{C}_{1}$ as a lower limit of concentration. Conversely, Region 2 is set as the positive side ( $\mathrm{x}>0$ ), with diffusion coefficient $\mathrm{D}_{2}$ and saturation $\mathrm{C}_{2}$ as the upper limit of concentration. Thus, after equilibrium at the interface is reached, concentration at $\mathrm{x}$ $=0^{-}$will be $\mathrm{c}_{1}$ and $\mathrm{x}=0^{+}$will be $\mathrm{c} 2$. This situation is similar to what was described during diffusion between the liquid and solid phases. However, in this analytical solution, no phase change or boundary movement are considered, saturation compositions will result only in limiting the diffusion rate.

Figure 3-8 shows the results and relative error for the analytical solution and model calculation for the above described case with $\mathrm{D}_{1}=4 * \mathrm{D}_{2}, \mathrm{c}_{1}=0.8, \mathrm{c} 2=0.4$ and $\sqrt{D_{1} t}=1$.

Just as observed in the finite/semi-infinite solid diffusion, the error peak is located in the region with the highest concentration gradient, and, just as before, the maximum error is directly proportional to $\Delta \mathrm{x}$. The same behavior with respect to the diffusion time is observed, where the error decreases with an increase in time, due to the decreased slope of the concentration curve.

\subsection{Modelling Heat Treatment Process Behavior}

As indicated earlier, the heat treatment of the $\mathrm{Cu}$-Sn system under analysis can be divided into five stages. The first stage occurs between room temperature and the melting point of Sn. The low diffusion rate and the short time duration of this stage results in no significant diffusion, and therefore, the first stage will not be considered for the modelling. 

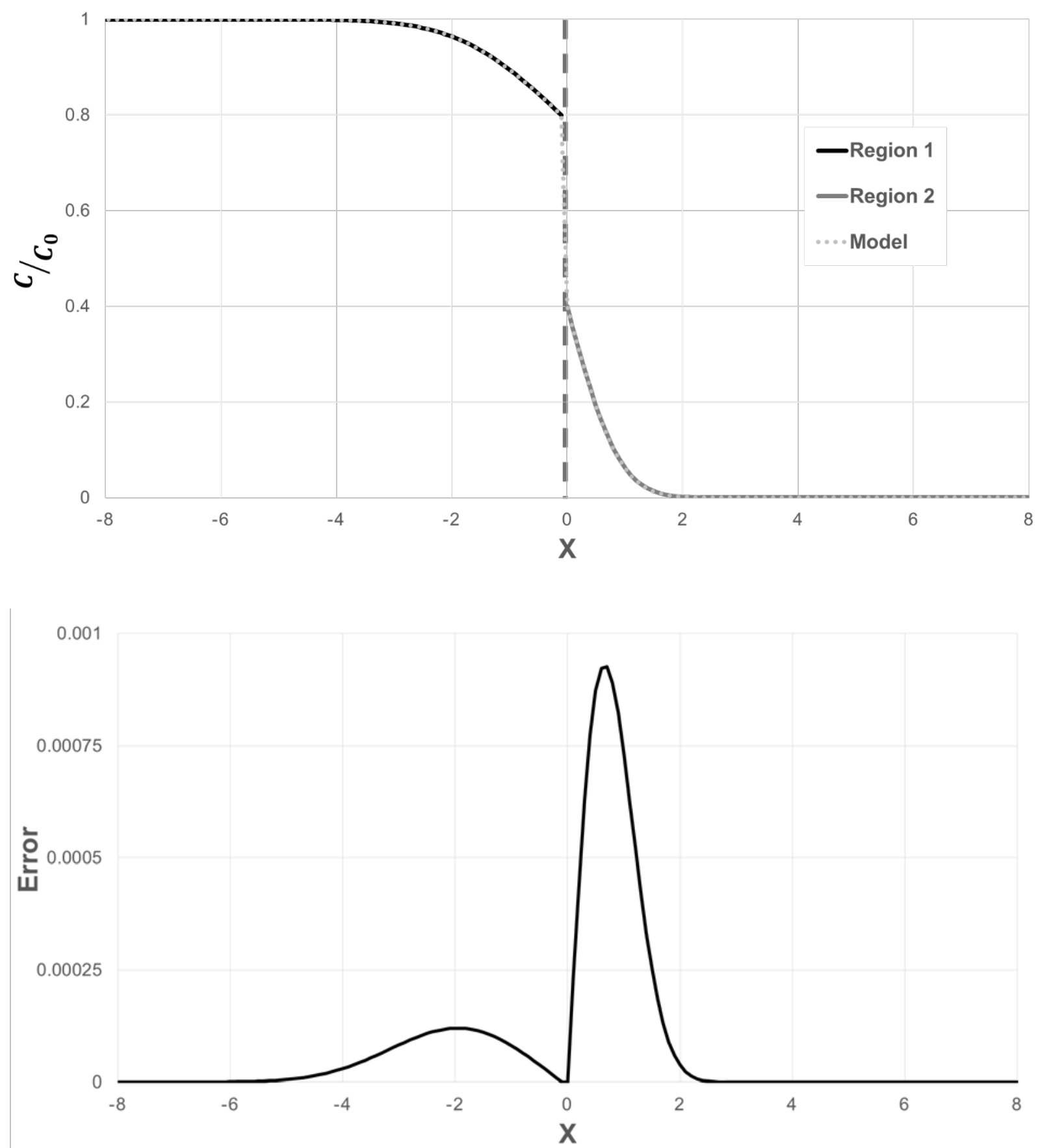

Figure 3-8 - Analytical and model solution for semi-infinite composite medium diffusion. 


\subsubsection{Second and Third Stages}

The second stage occurs during the heating of the system, from melting of the Sn coating until the working temperature is reached. Thus, its contribution to the overall diffusion process is highly dependent on the heating rate, which will define the shape of the temperature curve. The third stage is the same physical process as the second, albeit at a constant temperature. Consequently, both will be treated as one stage by the model. As mentioned before, other possible phases that form during these two stages are not considered in the present model.

For the solid portion of the system, a diffusion coefficient extrapolated from Hoshino et al. [28] will be utilized. The composition dependence is determined in accordance with the reported activation energy and frequency factor, and temperature dependence with the Arrhenius equation.

For the liquid phase, the diffusion is many orders of magnitude faster than that observed in the solid phase; therefore, the composition profile can be taken as homogeneous when viewed from the time frame of the solid diffusion. At the interface, the diffusion is limited by the slower region, which takes place in the solid. This reflects on the diffusion coefficient which, as explained before, is determined by the harmonic average, thus limiting the influence of the larger half, as can be seen in Figure 3-9. An increase of the larger coefficient becomes negligible when it is roughly 100 times the smaller coefficient, since further increases have no impact on the harmonic average. As the liquid self-diffusion of $\mathrm{Sn}$ is between 500 and $10^{13}$ times greater than the solid diffusion of Cu-Sn [27], it can be assumed that the mixture will remain at a similar order of magnitude. Thus, any number sufficiently large can be taken as the diffusion coefficient of the liquid phase. 


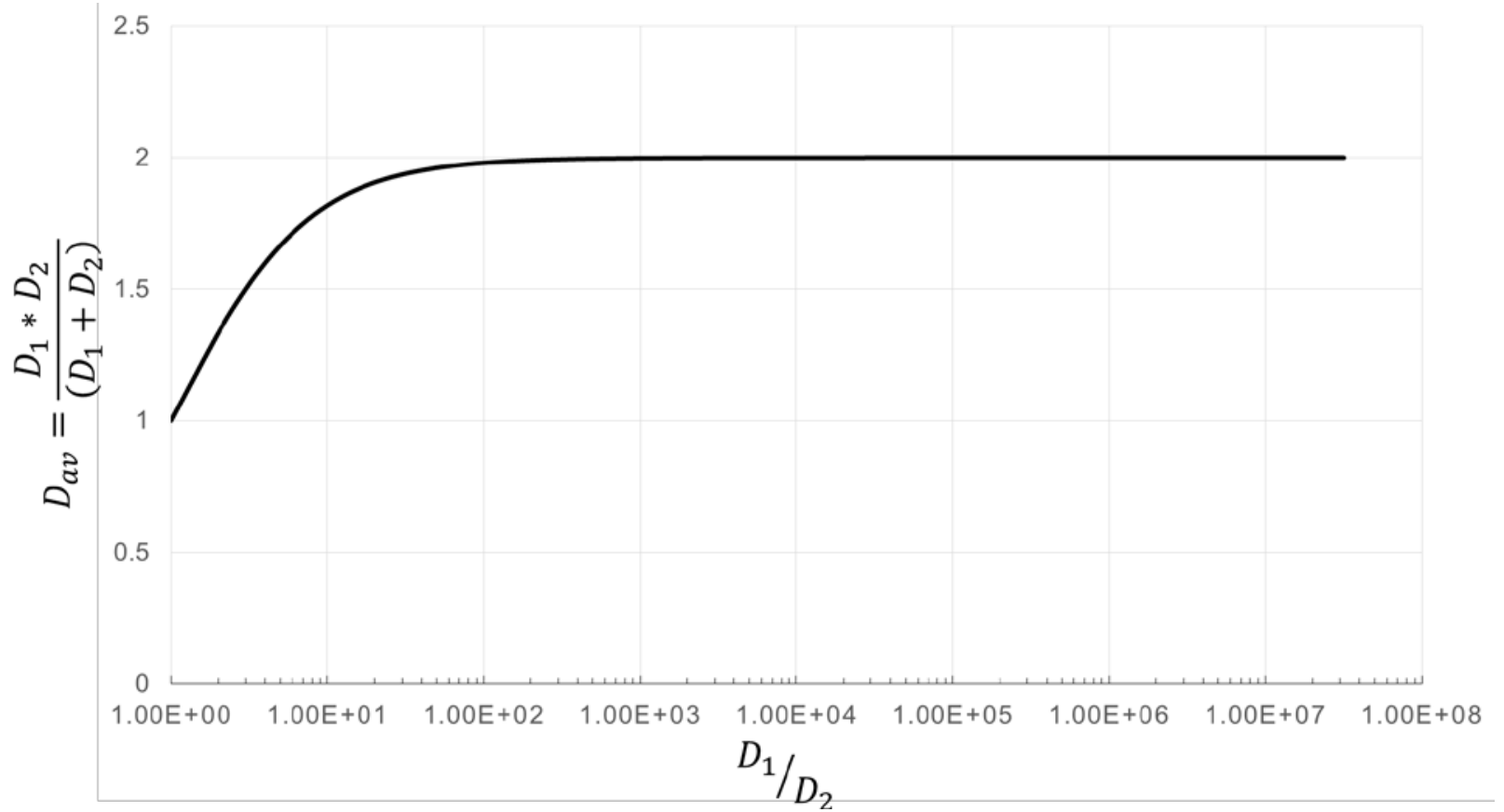

Figure 3-9 - Harmonic average for D1 and D2 at different ratios. 
To assess the impact of these stages on the overall diffusion process, the following temperature profiles were analyzed: constant temperature equal to the heat treatment temperature; reaching the target temperature for heat treatment in 30 seconds; reaching the target temperature in 120 seconds; and reaching the target temperature in 300 seconds; all with a logarithmic temperature increase. Each profile was analyzed for an annealing temperature of $800^{\circ} \mathrm{C}, 850^{\circ} \mathrm{C}$ and $900^{\circ} \mathrm{C}$. Other parameters used for the simulation were: initial Sn thickness of $2 \mu \mathrm{m}, \Delta \mathrm{x}$ of 0.1 $\mu \mathrm{m}$, and maximum $\Delta \mathrm{t}$ of $0.001 \mathrm{~s}$.

The results for the simulation of the moving boundary position and composition profile after the end of the third stage of heat treatment at $800^{\circ} \mathrm{C}$ are shown in Figure $3-10$ and Figure 3-11, respectively.

As expected, there is very fast movement of the boundary when the temperature is held constant. Solid dissolution starts almost immediately and saturation of the liquid phase happens after approximately 17.4 seconds with a thickness of $13.3 \mu \mathrm{m}$.

The temperature profile where the target temperature is reached in 30 seconds has a similar behavior as that observed by holding the temperature constant; however, there is no appreciable solid dissolution for roughly 19 seconds before temperature reached $651^{\circ} \mathrm{C}$. Liquid saturation occurs after 42.9 seconds with the same liquid thickness of $13.3 \mu \mathrm{m}$.

The third temperature profile, in which the working temperature is reached in 120 seconds, demonstrates similar behavior. Solid dissolution begins after 67 seconds, which corresponds to a temperature of $601^{\circ} \mathrm{C}$. Just like the other two profiles, the final thickness of the liquid is $13.3 \mu \mathrm{m}$ but required 120 seconds. 


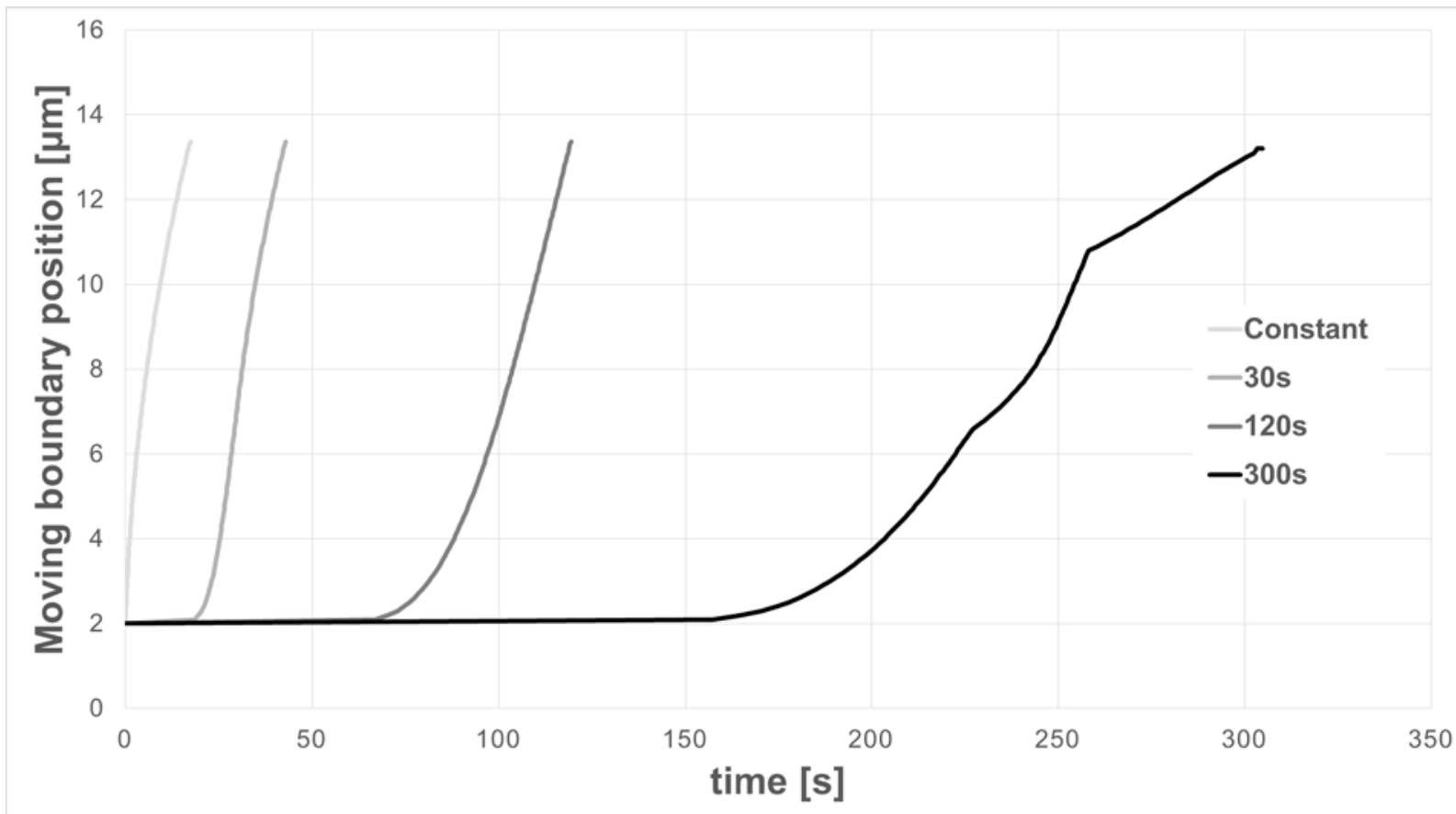

Figure 3-10 - Moving boundary position at the end of stage 3 of heat treatment at $800{ }^{\circ} \mathrm{C}$ for each temperature profile.

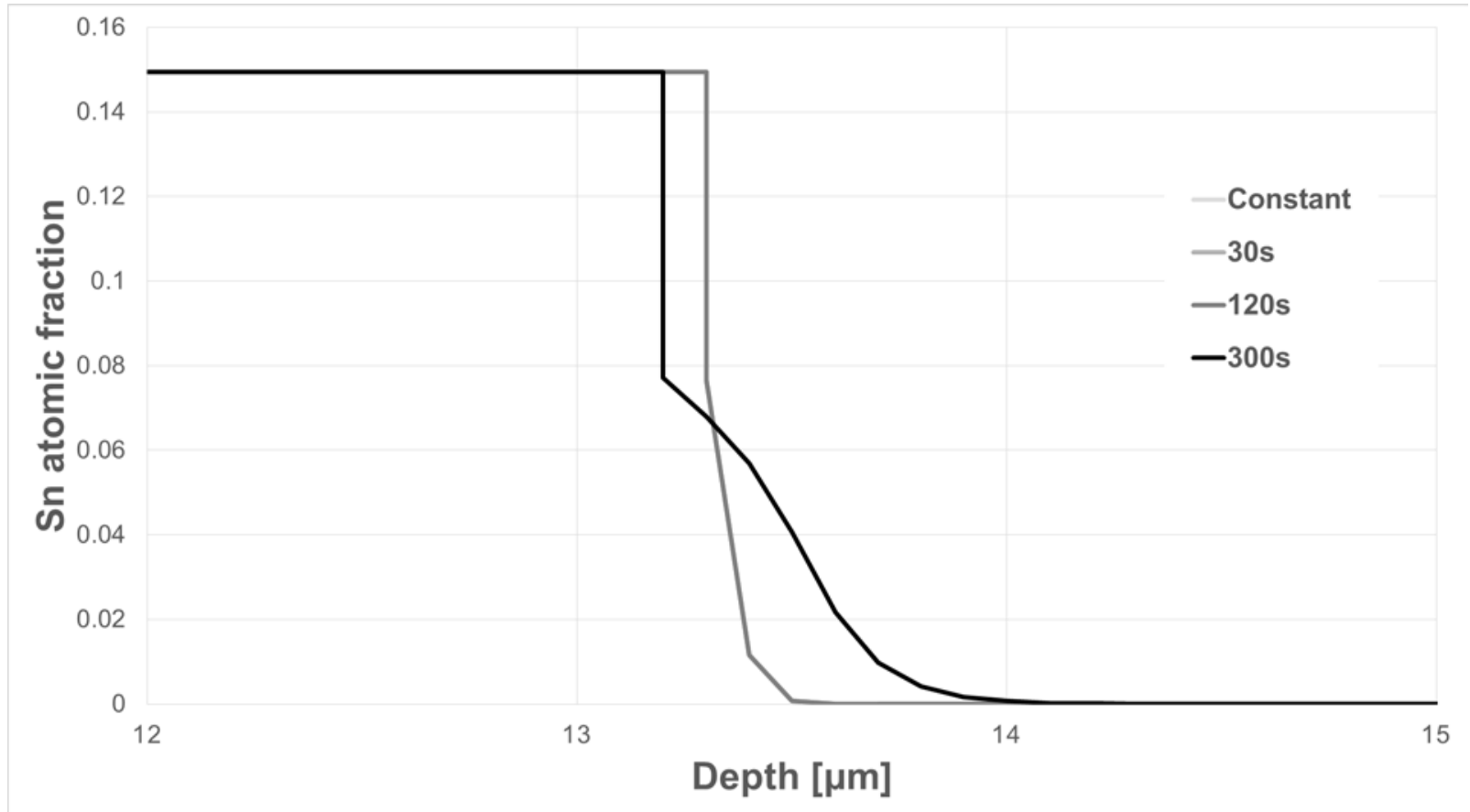

Figure 3-11 - Concentration profile at the end of stage 3 of heat treatment at $800{ }^{\circ} \mathrm{C}$, every profile except 300s is superimposed. 
The final profile, in which the target temperature is reached in 300 seconds, required 157 seconds to start the dissolution of the solid at a temperature of $574^{\circ} \mathrm{C}$. The slope of the boundary movement curve is smaller compared to the other profiles due to the reduction in heating rate. This creates the situation where there is enough time for the liquid phase to reach saturation at temperatures below the working temperature, which further limits boundary movement. Liquid saturation in the working temperature is reached shortly after working temperature itself is reached, or 304 seconds. However, the final thickness of the liquid is less than that obtained by the other profiles, $13.2 \mu \mathrm{m}$.

Diffusion at the liquid/solid interface is much higher than in the solid. Not only is the diffusion coefficient twice as large (due to the harmonic average), but the concentration gradient is also more pronounced, which is at least equal to the difference between liquid and solid saturation. For this reason, an insubstantial amount of diffusion occurs between two solid control volumes, since when a meaningful concentration gradient is established, enough diffusion occurred at the liquid solid interface to cause a melting phase transformation. However, when the liquid phase reaches saturation, there is enough time for solid diffusion to take place, as can be observed on the composition profiles shown in Figure 3-11, thus limiting the maximum liquid layer reached.

The simulation results for heat treatment temperatures of $850^{\circ} \mathrm{C}$ and $900^{\circ} \mathrm{C}$ are provided in Figure 3-12 to Figure 3-15. Just as observed for a heat treatment temperature of $800^{\circ} \mathrm{C}$, the liquid phase reaches liquidus composition below the targeted temperature with the heating profile of 300 seconds, which leads to solid state diffusion. However, unlike what was observed before, with the heating profile of 120 seconds, the same behavior is observed, also leading to solid state diffusion. This is not observed with the heating profile of 30 seconds. 


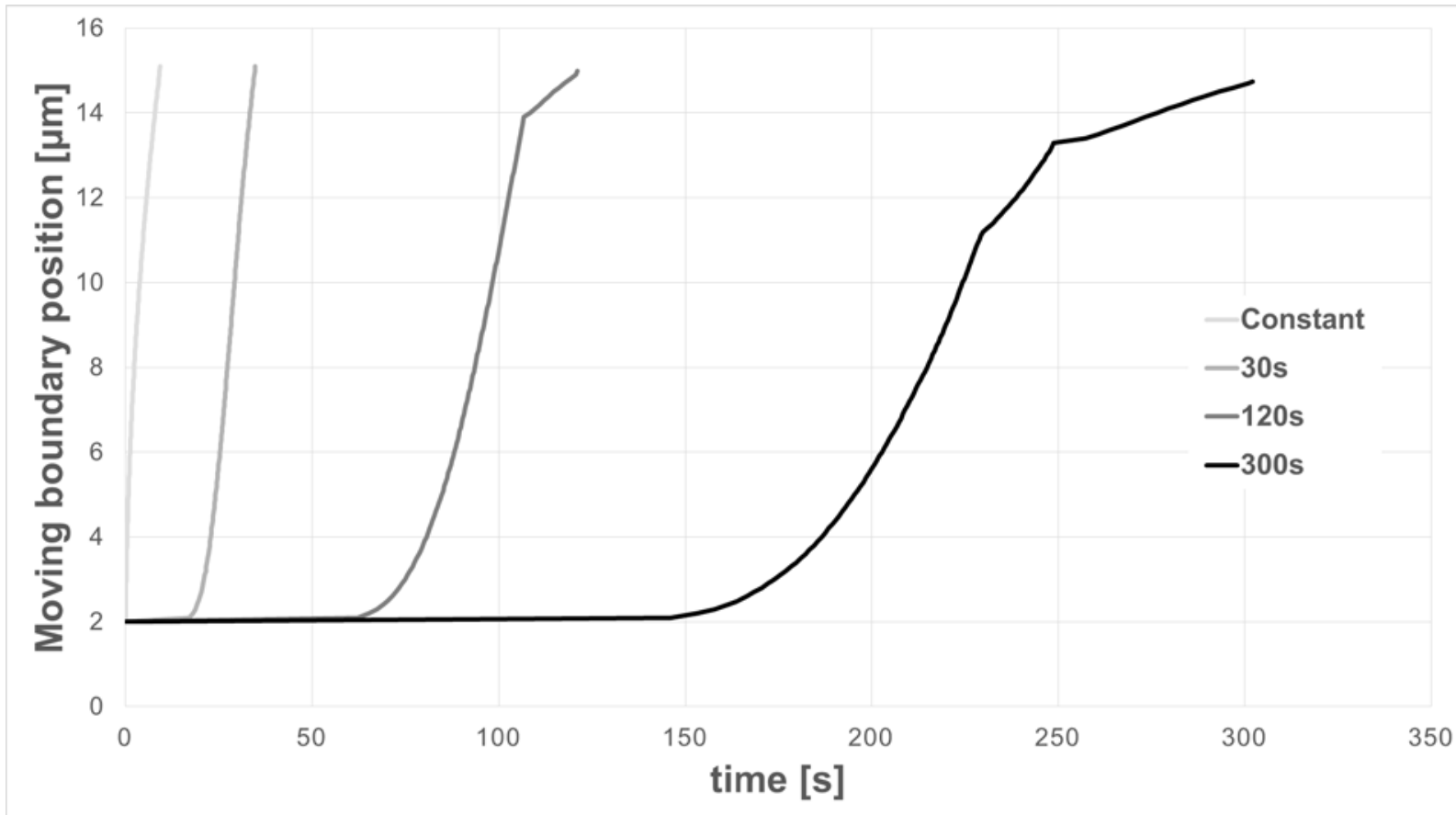

Figure 3-12 - Moving boundary position at the end of stage 3 of heat treatment at $850{ }^{\circ} \mathrm{C}$ for each temperature profile.

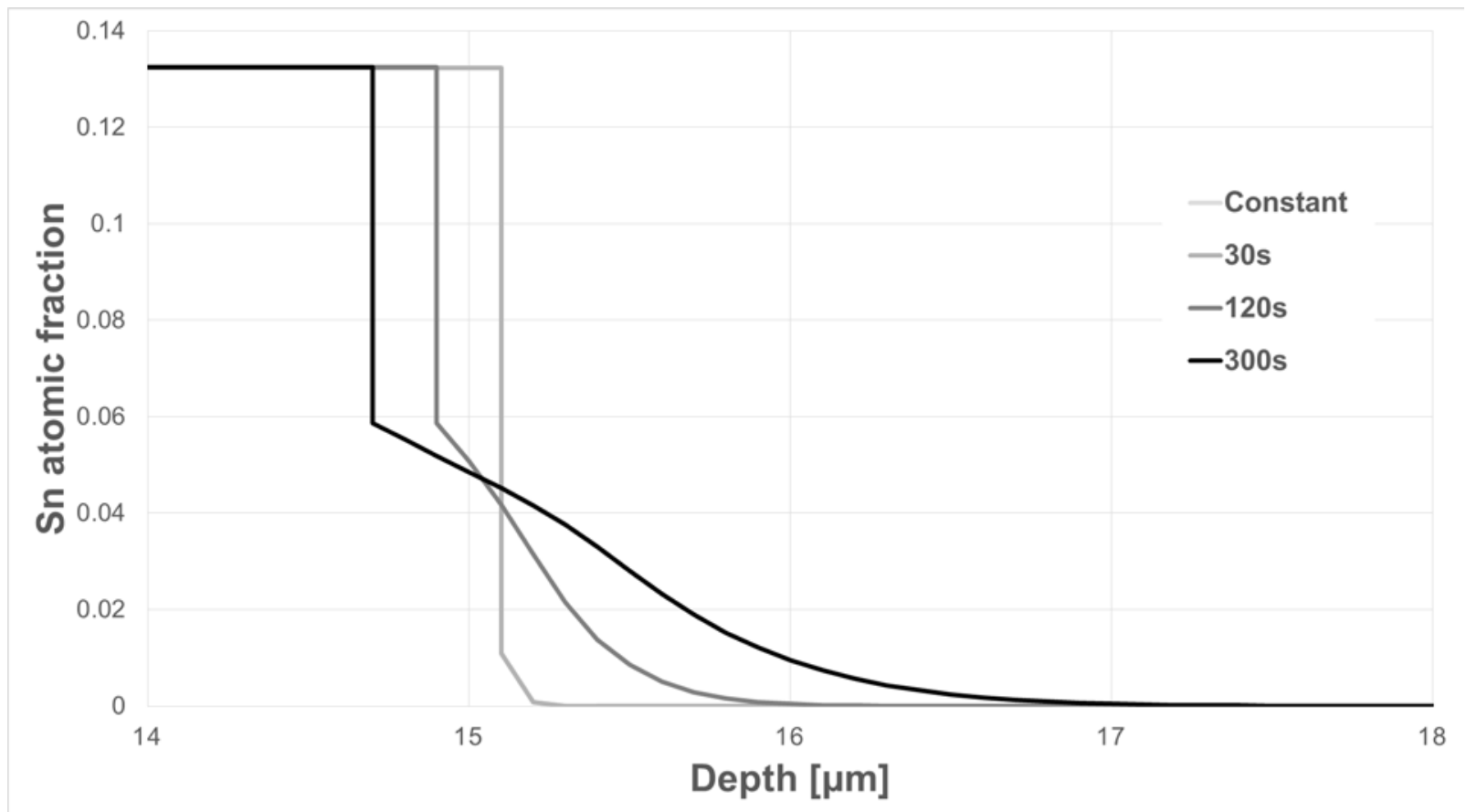

Figure 3-13 - Concentration profile at the end of stage 3 of heat treatment at $850{ }^{\circ} \mathrm{C}$, constant temperature and 30s heating profiles are superimposed. 


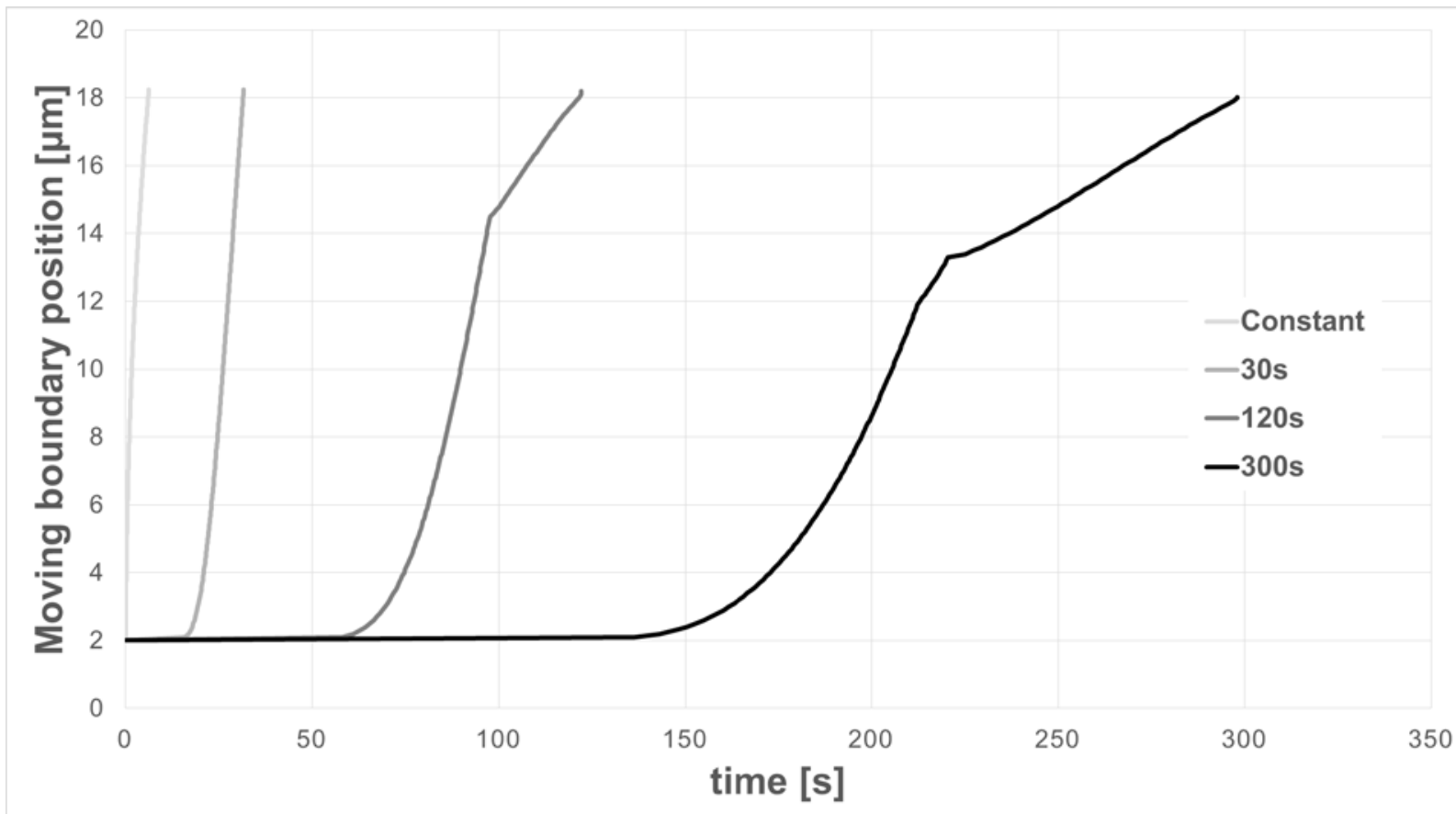

Figure 3-14 - Moving boundary position at the end of stage 3 of heat treatment at $900{ }^{\circ} \mathrm{C}$ for each temperature profile.

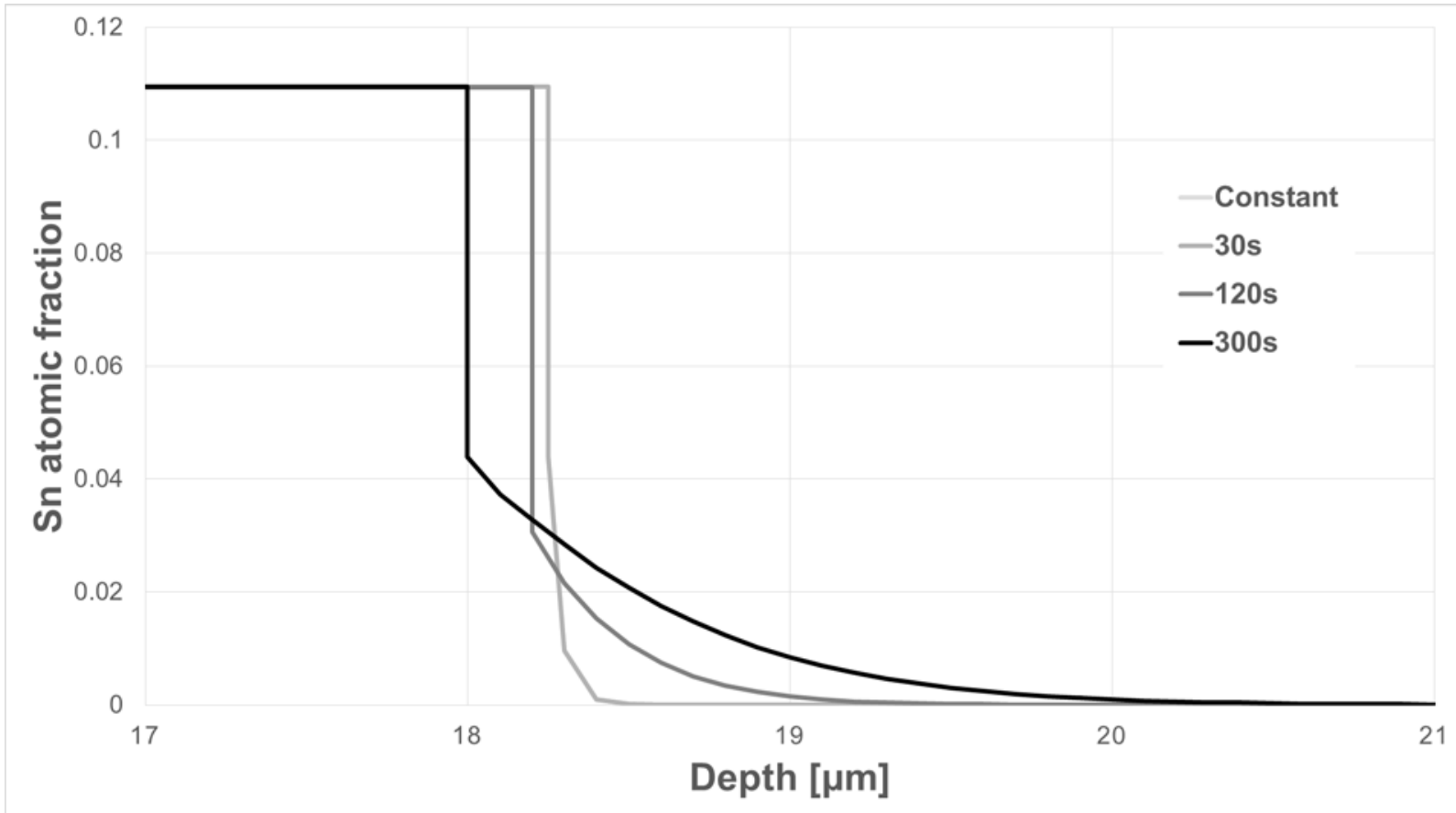

Figure 3-15 - Concentration profile at the end of stage 3 of heat treatment at $900{ }^{\circ} \mathrm{C}$, constant temperature and 30s heating profiles are superimposed. 


\subsubsection{Fourth Stage}

When the liquid phase reaches liquidus concentration, any further diffusion would cause it to solidify. However, on the other side of the interface lies an obstruction due to the fact that the solid phase cannot increase its concentration above solidus. In this stage, the rate of solid state diffusion dictates the speed of the process.

Simulation of the second and third stages showed that the composition profile is dependent on the heating rate, which can change the amount of solute that diffuse into solid, thus reducing the final thickness of the liquid. Figure 3-16 to Figure 3-18 show the boundary movement for different heating rates from the second to the fourth stage for heat treatment temperatures of $800^{\circ} \mathrm{C}$, $850^{\circ} \mathrm{C}$ and $900^{\circ} \mathrm{C}$, respectively. It is not surprising that for each heat treatment temperature, the order at which the simulated specimens reached complete solidification coincides with their initial heating rate. However, during the fourth stage, every curve appears to behave the same way regardless of the result of the third stage, thus indicating that the amount of diffusion that takes place during the second and third stages was insufficient to cause significant impact on the fourth stage. This is evidenced when time taken only in the fourth stage is compared; for instance, at $900^{\circ} \mathrm{C}$, the difference in the total time until solidification between the specimen that is heat treated at a constant temperature and that with a heating rate of 300 seconds is around 300 seconds, but the difference in the duration for the fourth stage alone is only 22 seconds.

The composition profiles obtained for each heat treatment temperature are shown in Figure 3-19. No appreciable difference can be identified between the profiles obtained by the different heating rates; therefore, all are represented as one. The aforementioned figure also illustrates the maximum possible surface concentration that can be obtained at each heat treatment temperature, which is determined by solid saturation. 


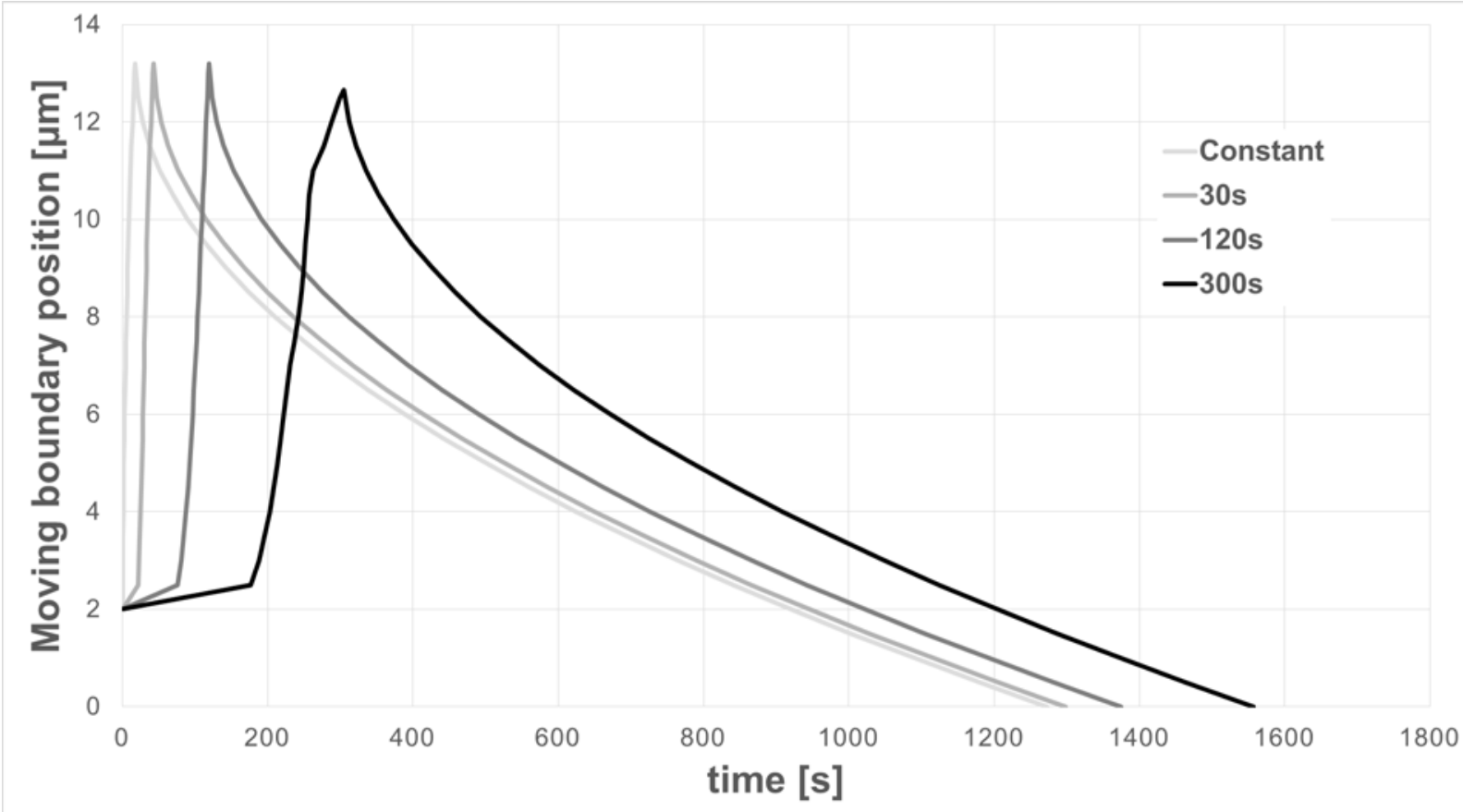

Figure 3-16 - Moving boundary position during stages 2-4 for heat treatment at $800{ }^{\circ} \mathrm{C}$ with different heating rates.

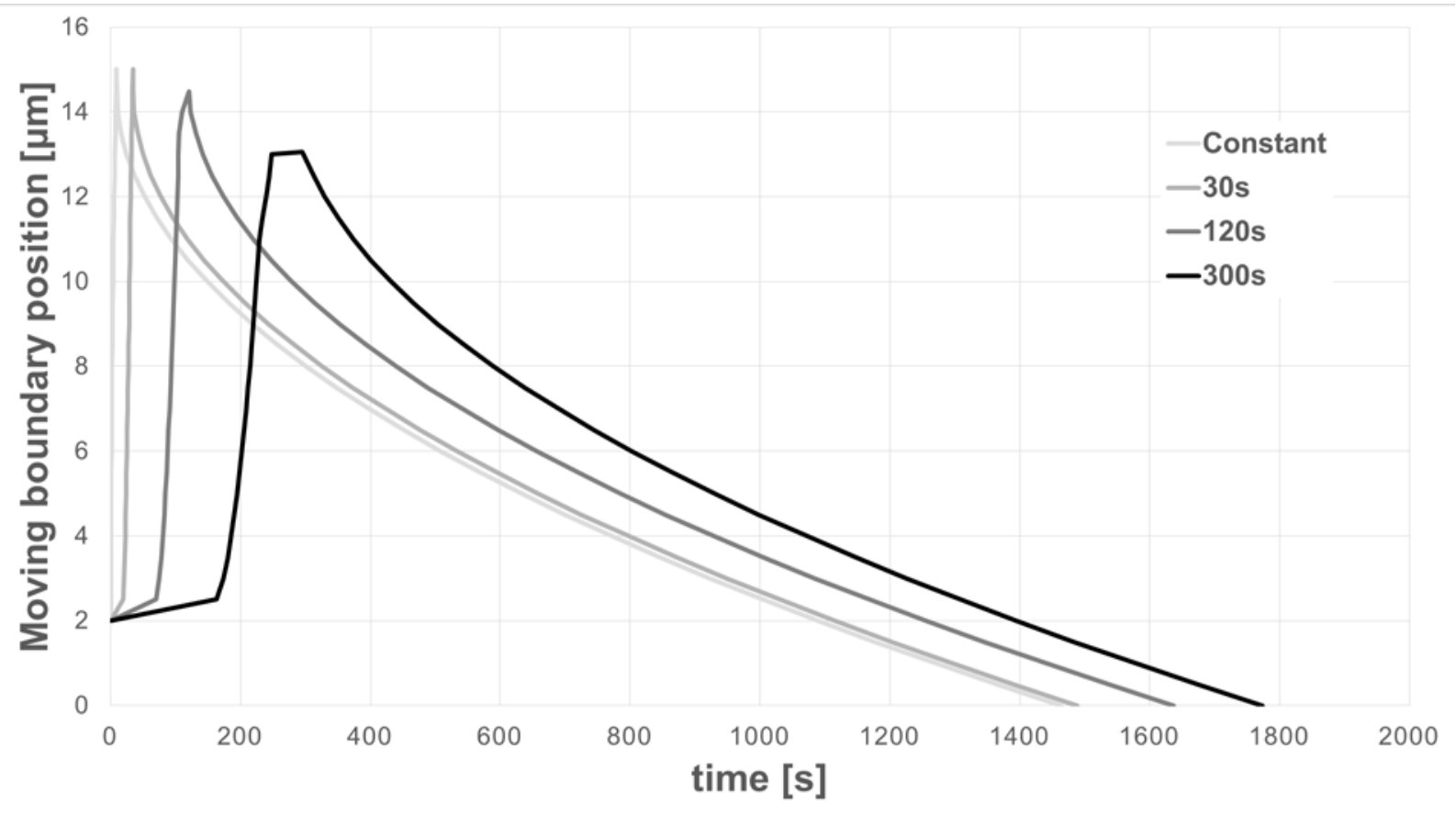

Figure 3-17 - Moving boundary position during stages 2-4 for heat treatment at $850{ }^{\circ} \mathrm{C}$ with different heating rates. 


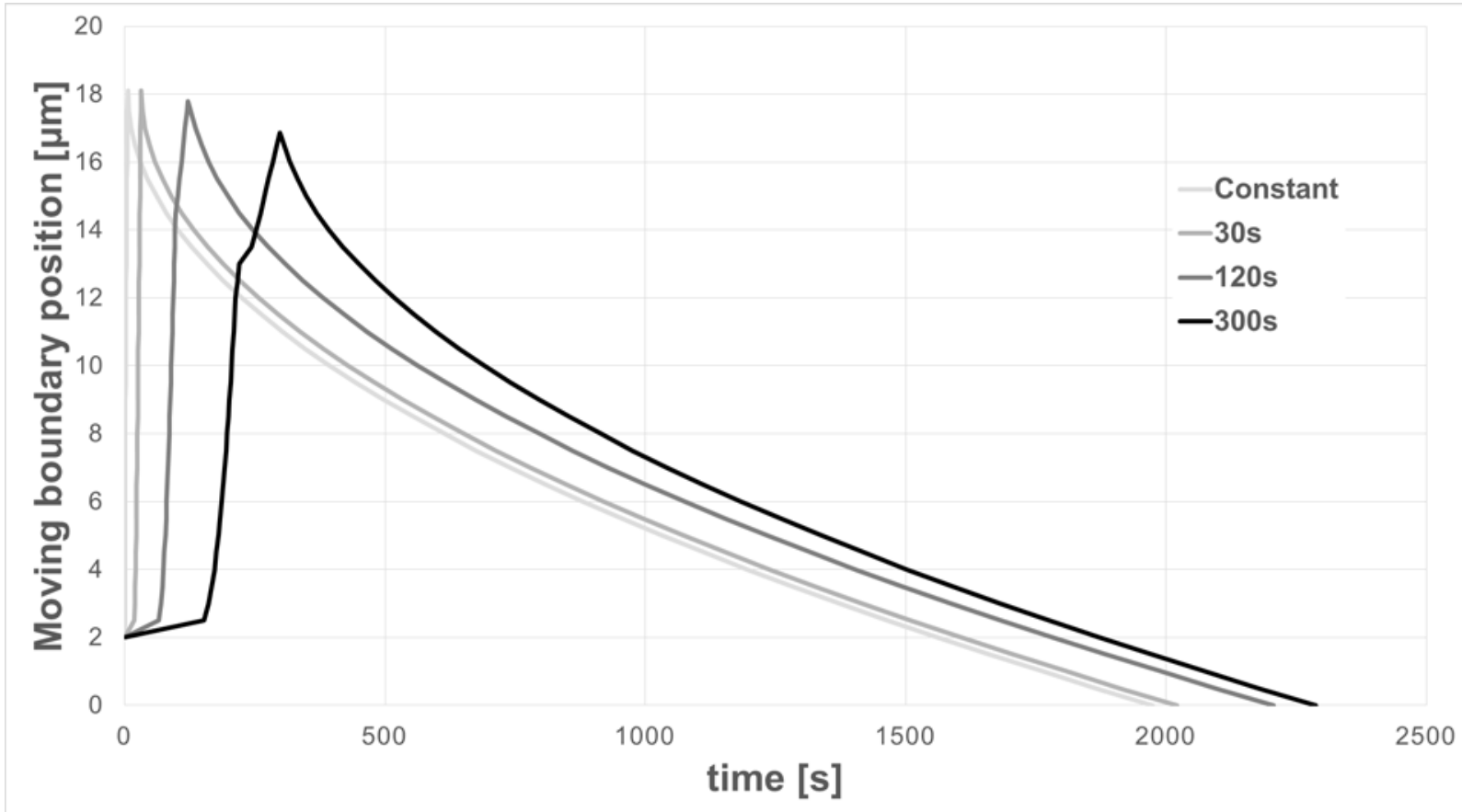

Figure 3-18 - Moving boundary position during stages 2-4 for heat treatment at $900{ }^{\circ} \mathrm{C}$ with different heating rates.

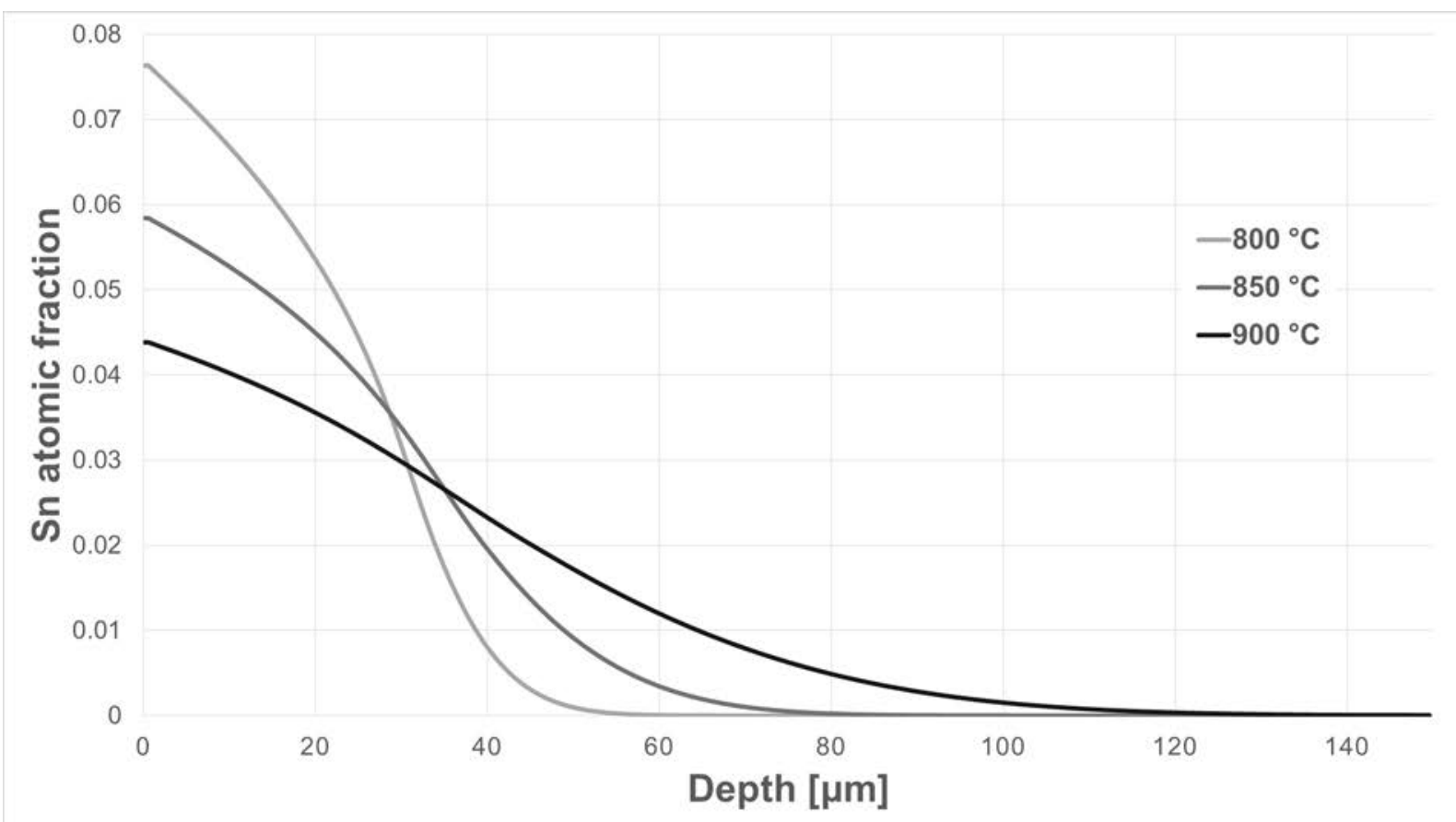

Figure 3-19 - Composition profiles after solidification for different heat treatment temperatures. 
By not considering the formation of other phases during the second stage, this creates a maximum possible diffusion of solute into the solid phase due to the artificially increased concentration gradient. During the fourth stage, these possible other phases would have dissolved due to the increased temperature, so their impact would be limited to the solute diffused into the $\alpha$ phase; therefore, the fact that no appreciable difference was observed at the end of the fourth stage between the analyzed temperature profiles indicates that the formation of other phases most likely would also not have any influence on those profiles.

In the temperature range under analysis, for a given amount of maximum liquid phase at the end of stage 3, complete solidification will be reached faster at higher temperatures, see Figure 3-20. This might seem logical since Arrhenius equation shows that the diffusion coefficient increases with temperature. However, for the case in this study, the maximum amount of liquid phase is not indicative of the actual amount of solute in it, since each temperature will be associated with a different saturation concentration. When comparing the same amount of $\mathrm{Sn}$ in the liquid phase, which is given by its initial thickness before heat treatment, solidification will take longer at higher temperatures, see Figure 3-21. This is caused by a decrease in solubility as temperature is increased. The decreased solubility leads to a smaller concentration gradient during solidification at higher temperatures, reducing the extent of diffusion and resulting in longer solidification times. 


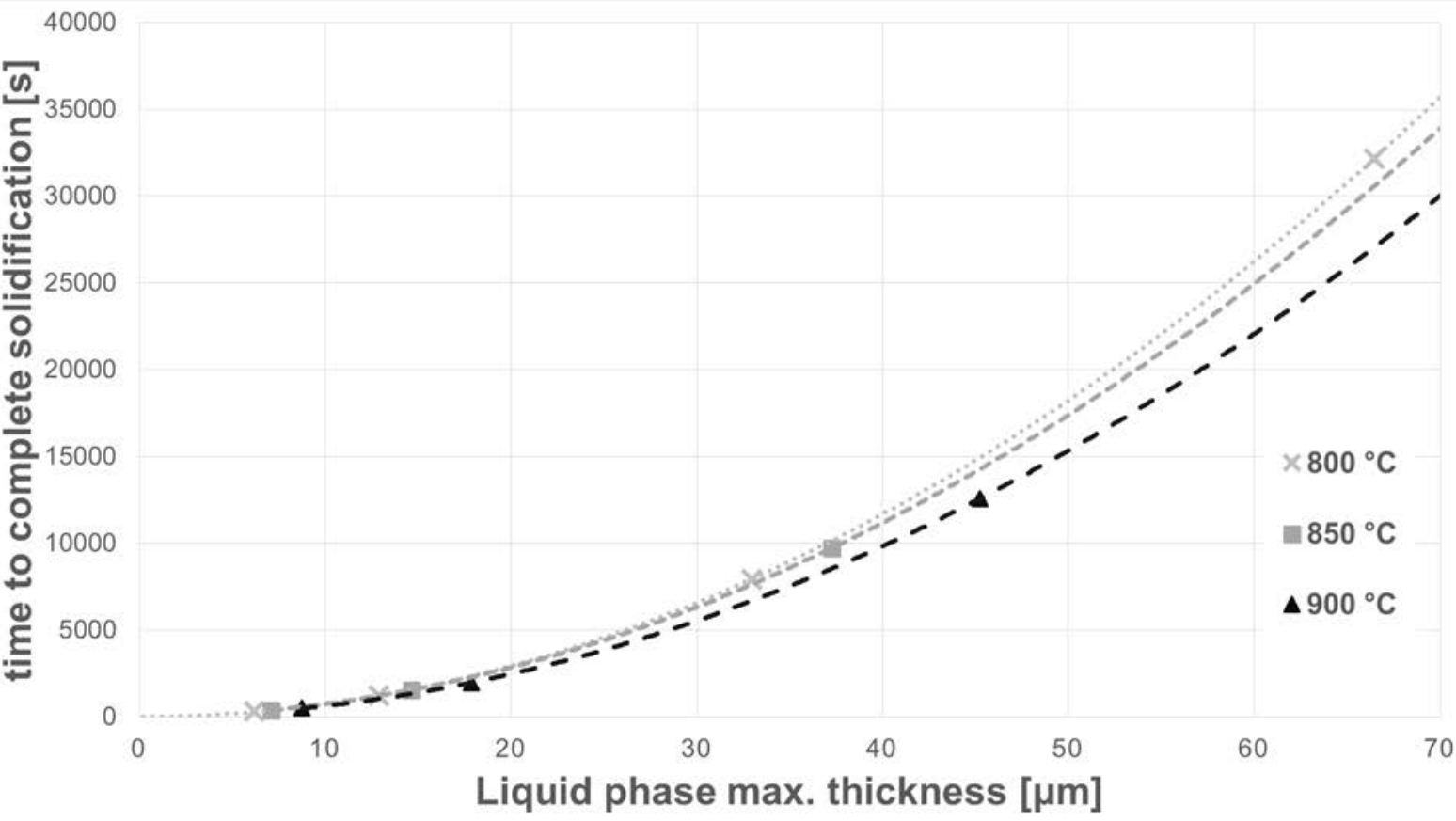

Figure 3-20 - Time to solidification of different liquid amounts for different temperatures.

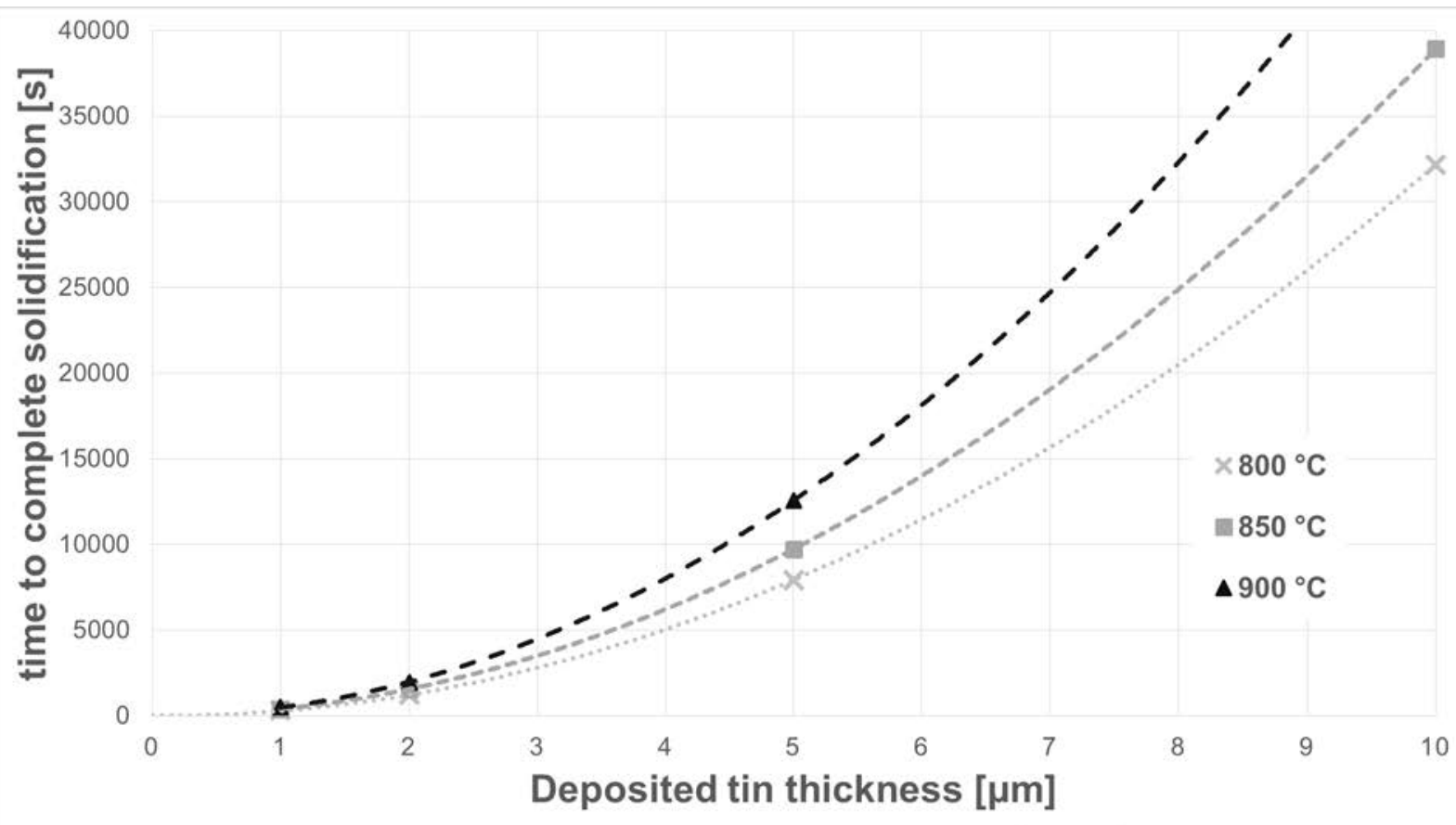

Figure 3-21 - Time to solidification of different deposited amounts for different temperatures. 


\subsubsection{Fifth Stage}

With the liquid phase completely solidified, the fifth stage begins, in which only solid state diffusion occurs. As time progresses, the concentration at the surface will only decrease. Figure 3-22 displays two curves obtained for the same surface concentration at different temperatures of $800^{\circ} \mathrm{C}$ and $900^{\circ} \mathrm{C}$. It can be seen that the solute content of the specimen that is heat treated at the higher temperature reaches deeper into itself at the expense of a lower concentration close to the surface, since total mass needs to be conserved. Conversely, with a lower temperature heat treatment, more $\mathrm{Sn}$ is found closer to the surface but compensated by a shallower diffusion depth. Therefore, the heat treating temperature becomes situational. If a higher concentration is desired and required throughout with a certain thickness close to the surface, a lower temperature would be ideal as it is capable of higher concentrations due to higher solid saturation, has a decreased slope in the composition curve, thus retaining the concentration deeper into the specimen; and offers more control over the desired surface composition due to the decreased diffusion rate. However, such advantages are time consuming. For instance, the simulated case of an Sn coating with a thickness of $2 \mu \mathrm{m}$ would require a heat treatment duration that is $38 \%$ longer at $800^{\circ} \mathrm{C}$ as opposed to $900^{\circ} \mathrm{C}$ to reach $6 \mathrm{Sn}$ at. $\%$ at the surface, and $140 \%$ longer at $800^{\circ} \mathrm{C}$ as opposed to $900^{\circ} \mathrm{C}$ to reach $4.8 \mathrm{Sn}$ at.\% at the surface. 


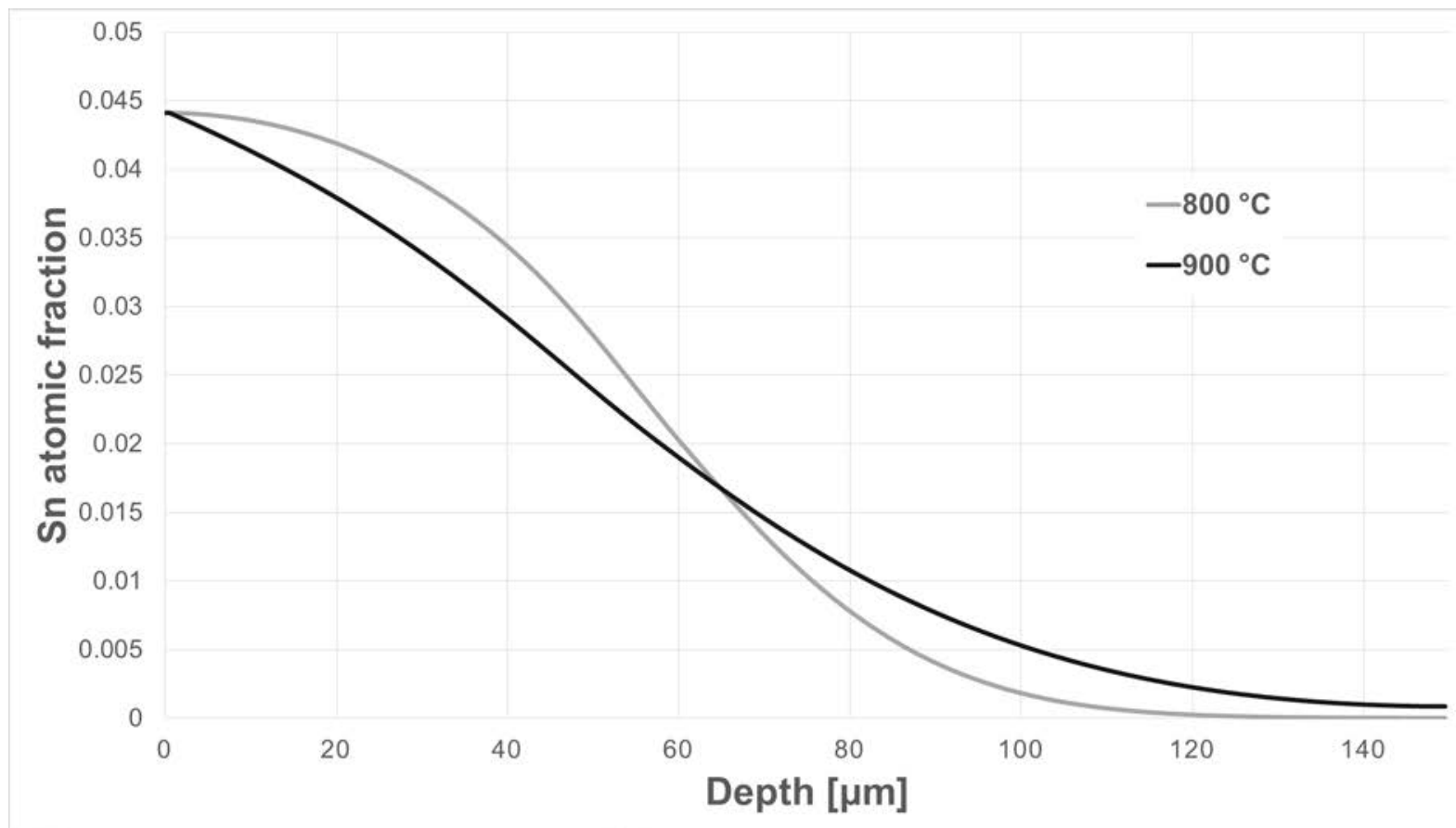

Figure 3-22 - Composition profiles of the same surface concentration obtained through different heat treatment temperatures. 


\subsection{Experimental Methodology}

Experimental verification was carried out to assess the validity of the model. The diffusion couple was composed of a Cu block, with dimensions of $10 \mathrm{~mm}$ x $10 \mathrm{~mm}$ x $4 \mathrm{~mm}$, coated with $\mathrm{Sn}$ through electrodeposition. The large difference in size between the thickness of Sn deposited and the $\mathrm{Cu}$ substrate ensures a semi-infinite condition, which means that the diffusion of $\mathrm{Sn}$ is not affected by boundaries or influenced diffusion from the opposite surface. However, the sample is still sufficiently small to quickly reach working temperature.

\subsubsection{Electrodeposition}

Electrodeposition was performed by using stannous sulfate as the main source of $\mathrm{Sn}^{2+}$ ions. Solid Sn electrodes were used to replenish the Sn ions as they were consumed. Gelatin was added in small quantities to improve the coating coverage, thus avoiding the formation of Sn whiskers during deposition, and also acted as a slight inhibitor of hydrogen evolution [42]. To increase the gelatin solubility, $\beta$-naphthol was added to the electrolyte. The list of chemicals and their concentrations are provided in Table 3-1.

The electrodeposition setup consisted of an electrochemical corrosion cell coupled with a Calomel reference electrode and a magnetic stirrer. The plating bath was maintained under an argon protected atmosphere. A VersaSTAT 3 galvanostat/potentiostat (Princeton Applied Research) was used as the power source.

The Cu samples were cut to size from a Cu ingot by using a Hansvedt Model DS-2 travelling wire electro discharge machine. After polishing, a thermocouple wire was spot-welded to one of the surfaces to serve as both an electrical connection and a sample holder during the electrodeposition. The thermocouple wire is thin, so its influence on the overall deposition process was minimal and the amount of Sn deposited onto the wire itself was negligible. 
Table 3-1 - Composition of stannous sulfate acidic electrolyte [1].

\begin{tabular}{|l|c|}
\hline \multicolumn{1}{|c|}{ Chemical } & $\begin{array}{c}\text { Concentration } \\
\text { (g/L) }\end{array}$ \\
\hline Stannous sulfate & 80 \\
\hline Sulfuric acid & 50 \\
\hline Phenolsulfonic acid & 40 \\
\hline Gelatin & 2 \\
\hline B-naphthol & 1 \\
\hline
\end{tabular}


Prior to the electrodeposition of Sn, the potential-current relationship of the plating bath was determined by using a voltammogram, as shown in Figure 3-23. Polarization started at $0.6 \mathrm{~V}$, sweeping towards the negative direction at a rate of $1 \mathrm{mV} / \mathrm{s}$. Between $0.10 \mathrm{~V}$ and $-0.47 \mathrm{~V}$, the current remained largely independent of the potential, which corresponds to the charging of a double layer [29]. As the potential became more negative, a distinct cathodic peak was observed, which indicated the reduction of $\mathrm{Sn}$ ions $\left(\mathrm{Sn}^{2+}\right)$ into metallic $\mathrm{Sn}\left(\mathrm{Sn}^{0}\right)$, effectively depositing them onto the surface. As the potential reached values below $-1.10 \mathrm{~V}$, a linear increase of the current began, thus signifying the start of hydrogen evolution [42].

The best deposition results were obtained through galvanostatic electrodeposition at 7.3 $\mathrm{mA} / \mathrm{cm}^{2}$, which resulted in a potential of $-1 \mathrm{~V}$. The deposition time was calculated by Faraday's law of electrolysis and actual thickness was determined by measuring the weight changes before and after deposition.

\subsubsection{Heat Treatment}

Prior to the heat treatment, the coated samples were individually placed into a quartz tube and submitted to at least three cycles of vacuuming by a diffusion pump up to $10^{-8}$ torr followed by fluxing with argon gas to remove any oxygen or moisture. After the final cycle, the quartz tube was filled with argon gas until ambient pressure, and enclosed by molding the glass tube with an oxy-propane torch, thus forming a protective capsule.

Heat treatment was performed on a Leco Laboratory Instruments box furnace. The furnace was always at the target temperature prior to sample insertion. After the heat treatment, the protective capsule around the samples was broken and they were quenched in water. Cold mounting with epoxy was then performed to avoid further diffusion. 


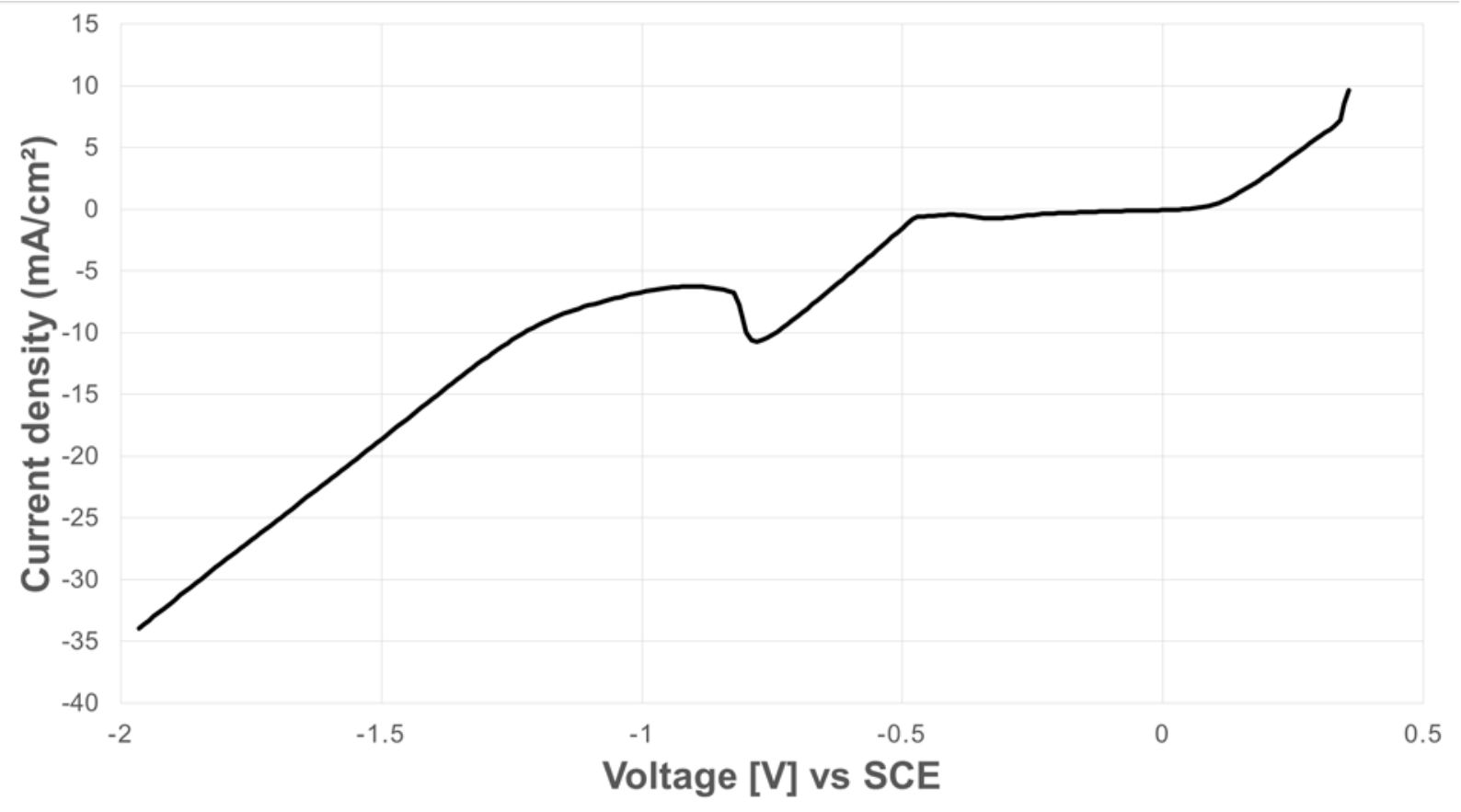

Figure 3-23 - Voltammogram of sulfate tin plating bath. 


\subsubsection{Scanning Electron Microscope Analysis}

Composition analysis was done with a JEOL JSM-5900LV scanning electron microscope (SEM) equipped with an Oxford Instruments energy dispersive spectral (EDS) analyzer, the INCA $\mathrm{X}$-Max detector. All of the measurements were taken at an accelerating voltage of $20 \mathrm{kV}$, and the focus was maintained at a working distance of $10 \mathrm{~mm}$. The EDS data were processed by using Oxford Instruments INCA Microanalysis Suite v.4.15 software. The experimental composition profiles of the samples were built by utilizing the point line scan tool from this software, with which the EDS analytical points were placed along a straight line with equal spacing. For the validation of the model, the composition profiles were measured at regions sufficiently away from the Sn-rich craters so that they would not affect the results. Both secondary electron (SE) images and backscattered electron (BSE) images were taken during analysis.

\subsection{Experimental Results and Comparison with Simulation Results}

The proposed model simulated the diffusion of $\mathrm{Sn}$ into $\mathrm{Cu}$ during heat treatment by assuming a constant density during the process. This assumption can be problematic when the initial conditions are set for the simulation process. The deposited solid Sn at the surface of the specimen assumes a $\beta$ Sn phase with a BCT crystal structure. After the heat treatment, $\mathrm{Sn}$ in solid solution with $\mathrm{Cu}$ assumes a FCC crystal structure, as it is now part of the $\alpha$ bronze phase. The volume and packing factor difference between the BCT and FCC crystal structures results in large density changes, such that the total mass of Sn before and after heat treatment, given by the integration of the Sn concentration curve, would not be constant if this aspect is not considered. To account for this, an equivalent deposition thickness is calculated, which represents the thickness Sn with a FCC crystal structure. 
Equation 3.22 is used to calculate the equivalent density:

Equation 3.22

$$
\rho^{\prime}=\frac{W * N}{n * a^{3}}
$$

where $\rho$ ' is the equivalent density; $W=118.7107$ [g/mol] is the atomic weight of Sn [43]; $N=4$ which is the number of atoms contained in the unit cell of the FCC; $n=6.022140857 \times 10^{23} \mathrm{~mol}^{-}$ ${ }^{1}$ ] which is the Avogadro constant [44]; and $a$, which varies between 3.68902 and 3.7179 [ $\AA$ ], is the lattice parameter of the $\alpha$ bronze [5]. By substituting these values, the equivalent density lies between 15.343 and $15.706\left[\mathrm{~g} / \mathrm{cm}^{3}\right]$. Taking the $\beta S n$ density as $7.365\left[\mathrm{~g} / \mathrm{cm}^{3}\right]$, the equivalent deposition thickness is about half (46.9\% 48.0\%) that of the actual deposited thickness.

Using the sample preparation described, a series of samples were prepared to validate the accuracy of the model. The parameters utilized for the samples are listed in Table 3-2. 
Table 3-2 - Parameters used for samples.

\begin{tabular}{|c|c|c|c|c|}
\hline$\#$ & $\begin{array}{c}\text { Actual deposited } \\
\text { Sn thickness } \\
{[\mu \mathrm{m}]}\end{array}$ & $\begin{array}{c}\text { Equivalent Sn } \\
\text { thickness } \\
{[\mu \mathrm{m}]}\end{array}$ & $\begin{array}{c}\text { Temperature } \\
{\left[{ }^{\circ} \mathrm{C}\right]}\end{array}$ & $\begin{array}{c}\text { Time } \\
{[\mathrm{min}]}\end{array}$ \\
\hline $\mathbf{1}$ & $7.0 \pm 0.8$ & $3.3 \pm 0.5$ & 850 & 60 \\
\hline $\mathbf{2}$ & $5.9 \pm 0.6$ & $2.8 \pm 0.4$ & 820 & 70 \\
\hline $\mathbf{3}$ & $6.0 \pm 0.5$ & $2.8 \pm 0.3$ & 870 & 80 \\
\hline $\mathbf{4}$ & $5.7 \pm 0.6$ & $2.7 \pm 0.4$ & 800 & 70 \\
\hline $\mathbf{5}$ & $5.5 \pm 0.8$ & $2.6 \pm 0.4$ & 890 & 80 \\
\hline $\mathbf{6}$ & $3.7 \pm 0.1$ & $1.7 \pm 0.1$ & 830 & 20 \\
\hline
\end{tabular}


Sample \#1 has the thickest deposition of Sn. For the sample size utilized, a Sn coating with a thickness of $7 \mu \mathrm{m}$ proved to be overly thick. During the heat treatment process, the liquid thickness reached the point where it could not be sustained by the surface tension. This resulted in liquid flowing towards the bottom side of the sample and creating two distinct surfaces at the end of the process: a top surface with a low content of Sn and the bottom surface with a layer of eutectics, which indicates that the liquid was still present during quenching.

Figure 3-24 and Figure 3-25 are BSE cross section images of the bottom and top surfaces, respectively, of Sample \#1 after the heat treatment process. EDS analysis of the features in the eutectic phase indicated a composition between 7 and 9 Sn at.\% for the darker regions, which is consistent with the $\alpha$ phase, and between 15 and 16 Sn at.\% for the brighter regions. The composition of the brighter regions is indicative of a $\beta / \gamma$-based metastable phase, since both $\beta$ and $\gamma$ phases are not found at room temperature [5]. This combination of phases indicates that the original liquid layer, prior to cooling, had a composition consistent with that of liquid saturation at the analyzed temperature range, which is in agreement with the described process. 


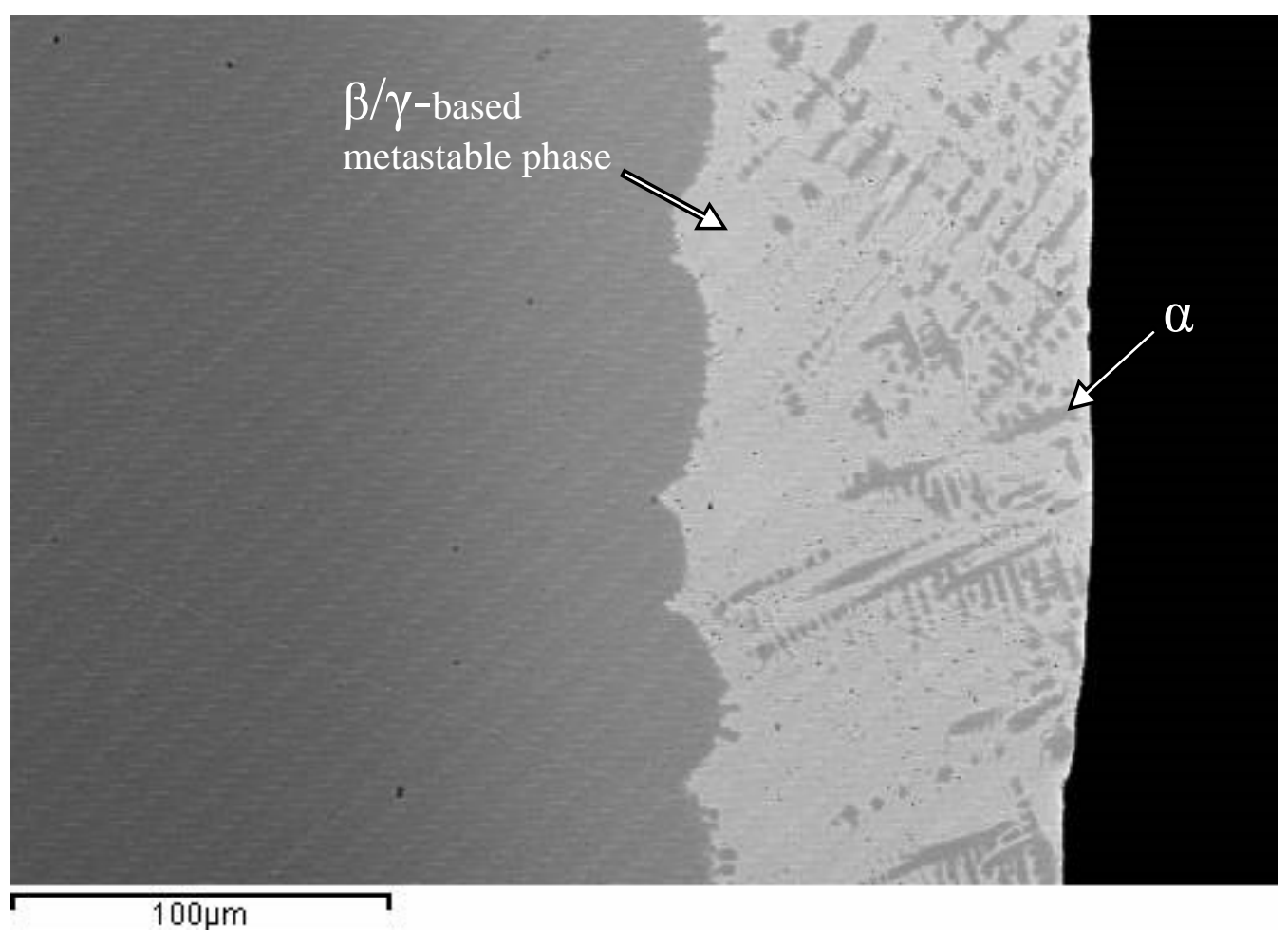

Figure 3-24 - Cross section BSE image of the bottom surface of sample \#1.

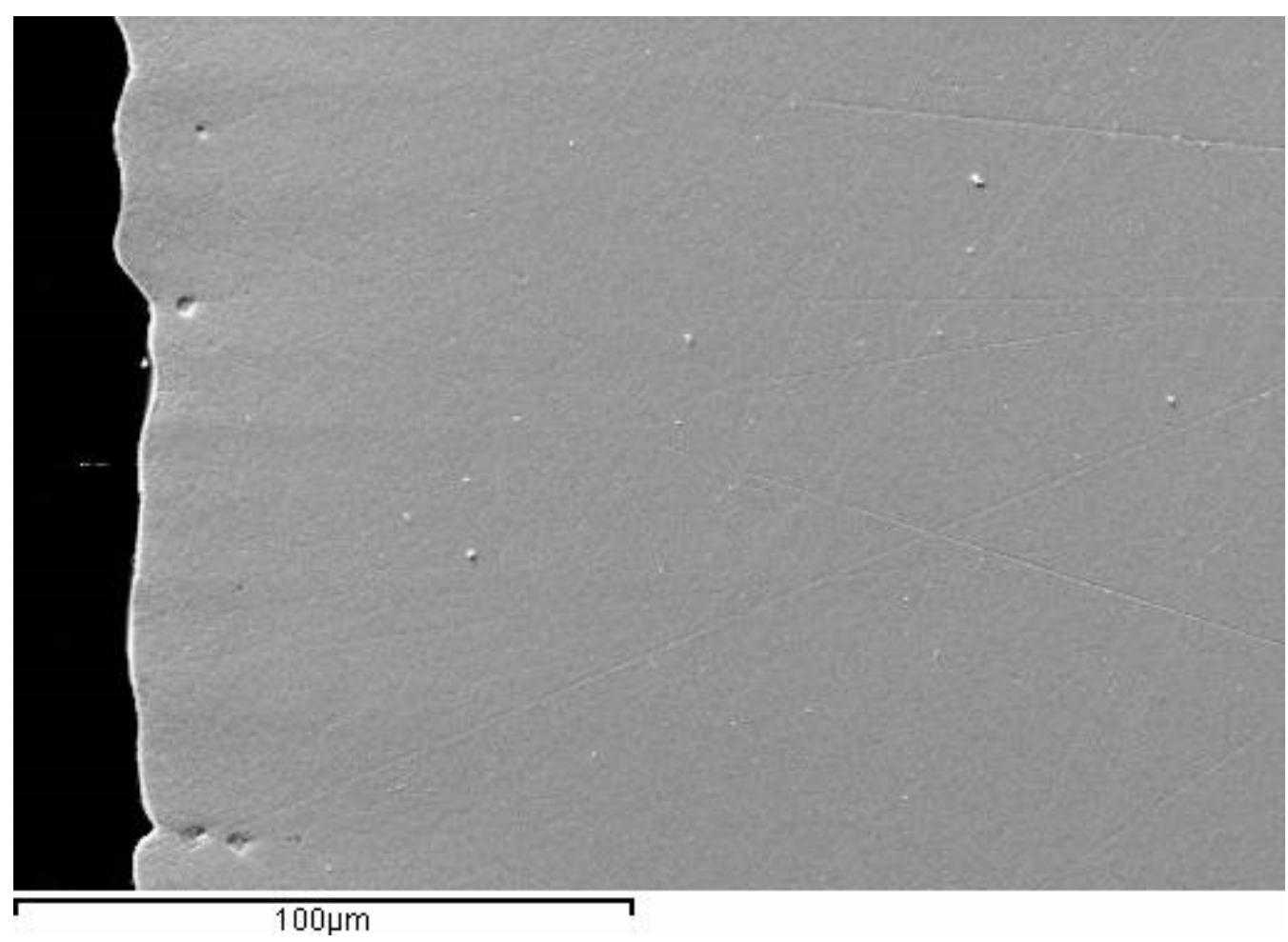

Figure 3-25 - Cross section BSE image of the top surface of sample \#1. 
Below the eutectic layer, the bottom surface never reached the fifth stage. This creates a simplified situation which can be used to check the model. In this condition, the highest concentration of Sn, found at the interface with the eutectic layer, is indicative of solid saturation concentration, which can be attributed to the actual heat treatment temperature. For Sample \#1, this is estimated to be $860^{\circ} \mathrm{C}$, which is equivalent to a solid saturation of $5.2 \mathrm{Sn}$ at.\%. By running the model with a temperature of $860^{\circ} \mathrm{C}$ and an initial thickness of $\mathrm{Sn}$ that is equivalent to the total Sn diffused below the eutectic, the time required by the fourth stage is determined to be $60.1 \mathrm{~min}$, which is virtually the same amount of time that the actual experimental specimen underwent heat treatment. Conversely, the same simulated profile could be obtained by running the simulation for the prescribed amount of time and a semi-infinite liquid layer, which allows the maximum amount of diffusion possible into the solid. The experimental and simulated composition profiles of the eutectic covered side of the sample are shown in Figure 3-26.

In a similar fashion, the opposite side of the specimen was simulated by using the same temperature and time as the model parameters but with a reduced Sn content, which was determined from the experimental sample. Unlike to other side, this surface underwent every stage of heat treatment, including the fifth stage of solid state diffusion. The simulated composition profile was found to be close to that experimentally observed, see Figure 3-27. 


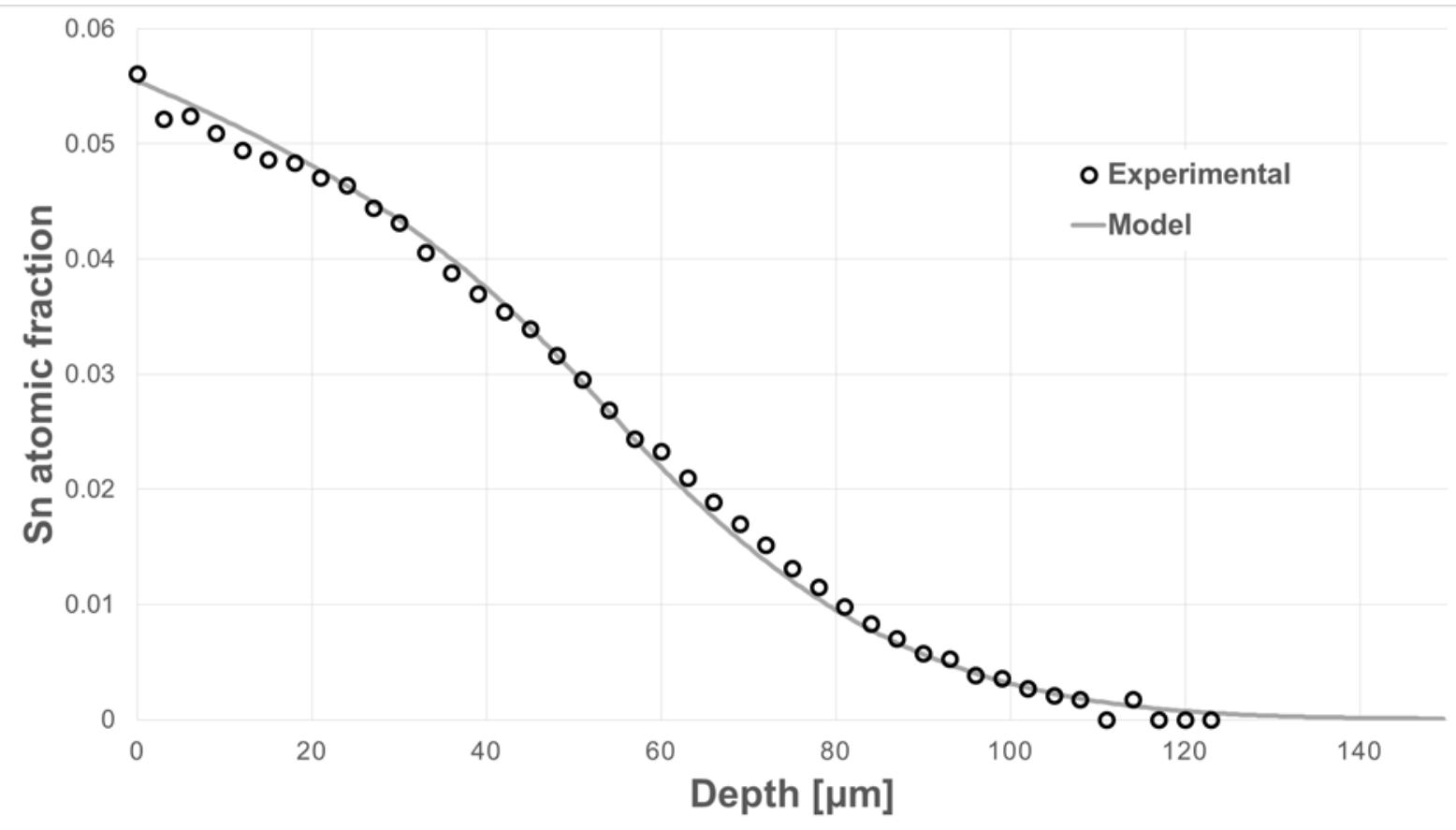

Figure 3-26 - Experimental data (circles) and model simulation (line) of composition profile below eutectic layer of sample $\# 1$ heat treated at $850^{\circ} \mathrm{C}$ for $60 \mathrm{~min}$. Simulation temperature $=860^{\circ} \mathrm{C}$.

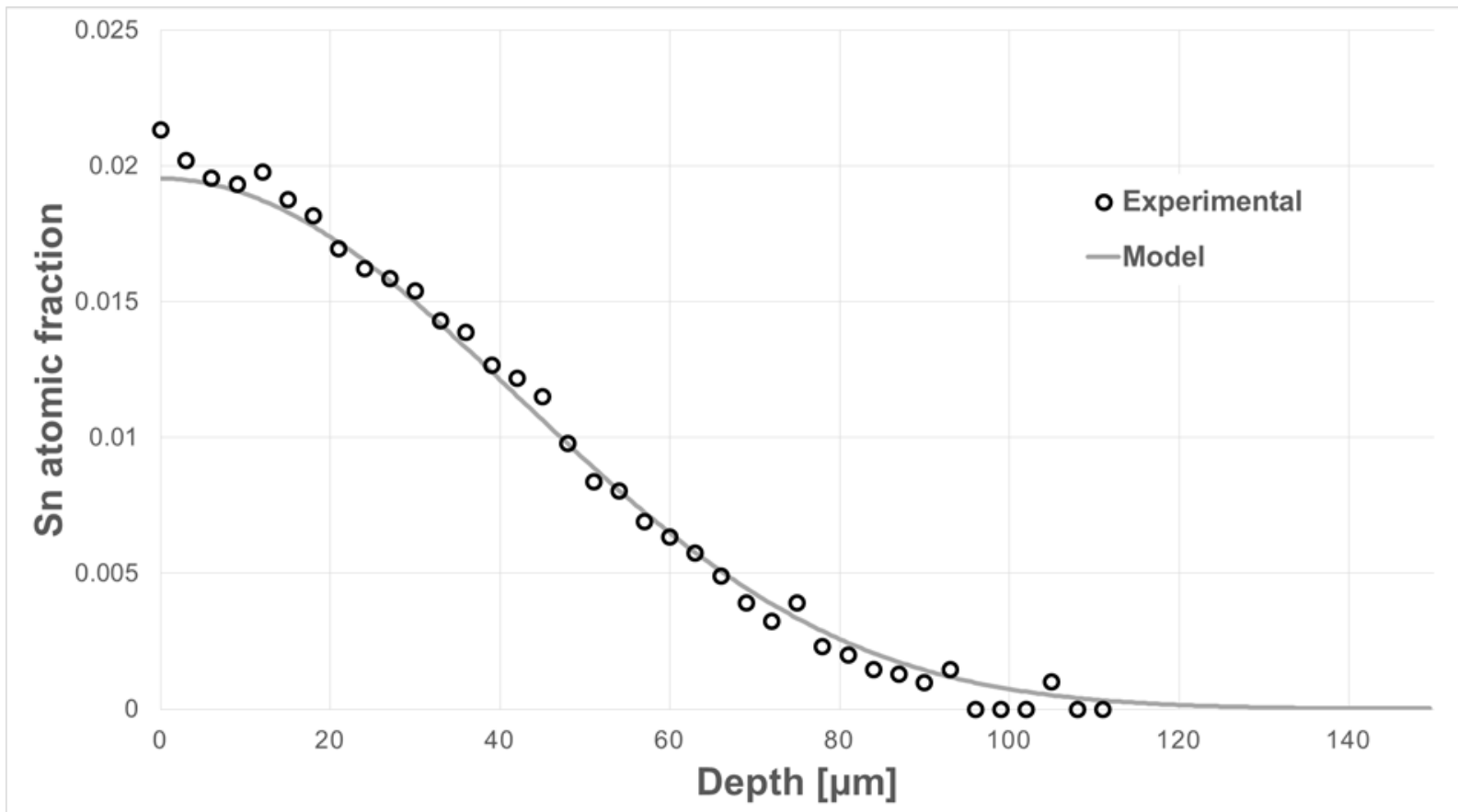

Figure 3-27 - Experimental data (circles) and model simulation (line) of composition profile on low tin content side of sample \#1 heat treated at $850{ }^{\circ} \mathrm{C}$ for $60 \mathrm{~min}$. Simulation temperature $=860{ }^{\circ} \mathrm{C}$. 
The data obtained from Sample \#1 can be used as a platform to demonstrate the importance of considering the dependence of the diffusion coefficient on the concentration. To simulate the eutectic covered surface, the model was adapted to take into consideration the case in which the diffusion coefficient is not dependent on concentration with the same parameters used previously. The diffusion coefficient value was determined as that with which the aforementioned mass diffused in 60 minutes, thus amounting to $0.438 \mu \mathrm{m}^{2} / \mathrm{s}$. Figure 3-28 shows the resultant simulated profile plotted against the same experimental data which was shown in Figure 3-26.

There is a clear departure between the two curves. The constant diffusion coefficient provides a simulated curve with a shape similar to exponential decay, whereas the experimental data displays a larger amount of solute close to the surface and lower away from it, which indicates that in reality, the diffusion coefficient indeed varies with composition. Using the same value for the diffusion coefficient not dependent on concentration $\left(0.438 \mu \mathrm{m}^{2} / \mathrm{s}\right)$, the other side of the sample was simulated, as shown in Figure 3-29. Unlike the observation for the modeling of the concentration dependent diffusion coefficient, the results of this simulation do not agree with the experimental data. The coefficient that was previously set to obtain the correct rate of diffusion on the eutectic side, completely overestimates the rate of diffusion when different conditions are simulated, in this case, with different initial masses of Sn. The diffusion coefficient which is considered independent of the composition is a simplification often taken when addressing diffusion problems and, indeed, in some situations it is reasonable. However, as shown, this assumption leads to errors that any prediction becomes null for the case in this study. 


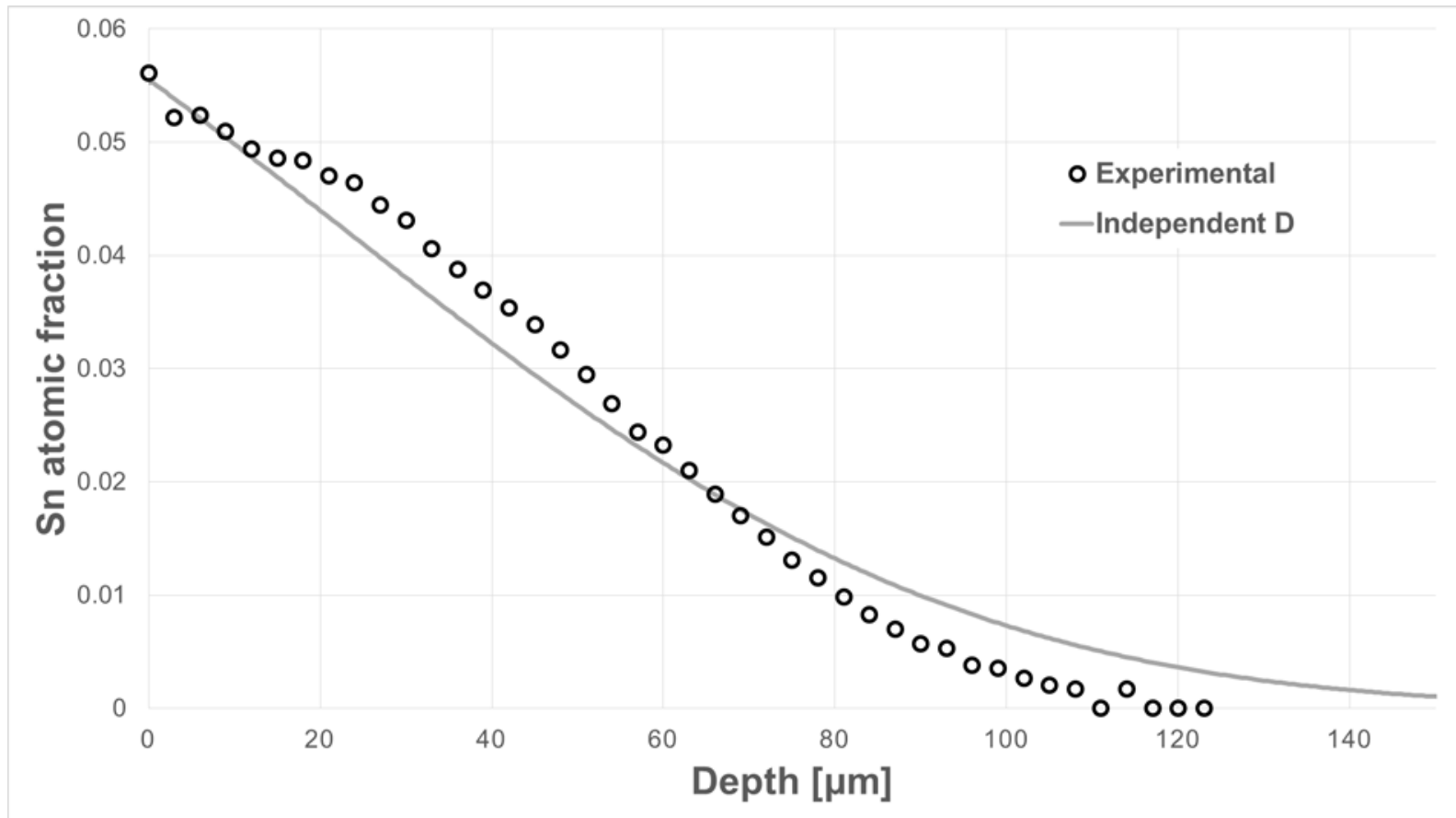

Figure 3-28 - Experimental data (circles) and model simulation with concentration independent diffusion (line) of composition profile below eutectic layer of sample \#1 heat treated at $850{ }^{\circ} \mathrm{C}$ for $60 \mathrm{~min}$. Simulation temperature $=860^{\circ} \mathrm{C}$.

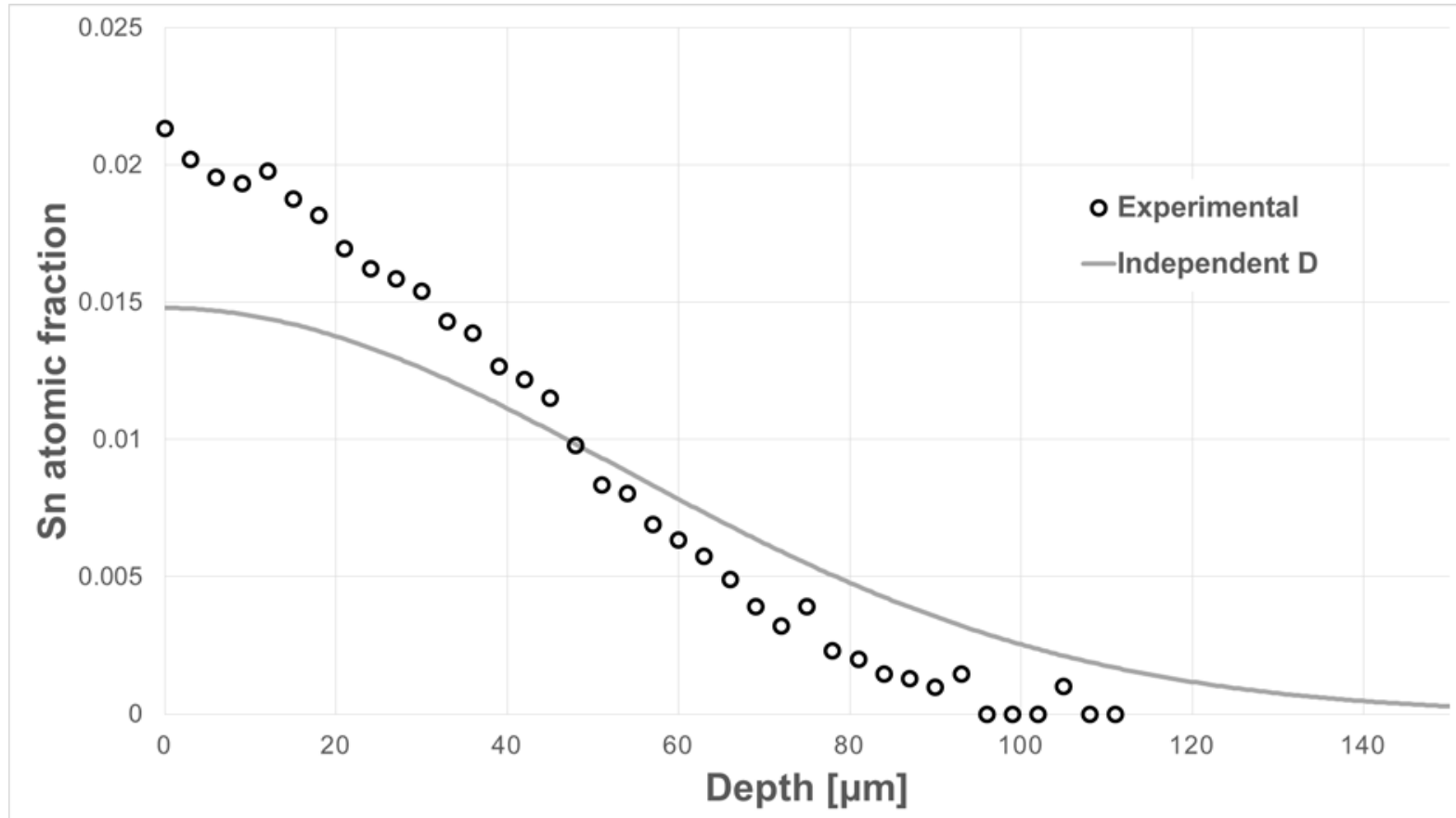

Figure 3-29 - Experimental data (circles) and model simulation with concentration independent diffusion (line) of composition profile on low tin content side of sample \#1 heat treated at $850{ }^{\circ} \mathrm{C}$ for $60 \mathrm{~min}$. Simulation temperature $=860^{\circ} \mathrm{C}$. 
The deposition layer on Sample \#2 was thin enough so that it did not display the same behavior observed on Sample \#1 during the heat treatment. For the simulation of this sample, the uncertainties around the deposition thickness and actual heat treatment temperature can affect the accuracy of the solution. By analyzing the experimental data of this sample, the equivalent deposition thickness was determined to be $2.45 \mu \mathrm{m}$, which is within the error of what was measured prior to the heat treatment. A series of profiles were simulated with the model, keeping all of the parameters the same except for temperature. Figure 3-30 shows the experimental data, simulation at $820^{\circ} \mathrm{C}$ (temperature given by furnace controller) and the best fit obtained by the simulation, at $840^{\circ} \mathrm{C}$. A better approximation of the experimental data from the simulation with a higher temperature indicates that there is in fact a slight offset between the temperature set in the furnace and the actual temperature imposed on the sample, which was also observed for Sample \#1.

Similar to Sample \#2, Sample \#3 did not have any overflow of Sn. However, the maximum thickness of the liquid phase in Sample \#3 should be comparable to that of Sample \#1, due to the approximate thickness of the deposition and the higher temperature that this sample underwent during the heat treatment. The fact that no overflow was observed suggests that such movement occurs as Sn melts, and therefore would be mainly affected the deposition thickness rather than the maximum liquid thickness.

The amount of Sn diffused on Sample \#3, as measured from the experimental data, has an equivalent deposit thickness of $2.29 \mu \mathrm{m}$, or $0.24 \mu \mathrm{m}$ below the expected lower limit. The experimental and simulation composition profiles are provided in Figure 3-31. Once again, the best fit is obtained at a higher simulated temperature of $880^{\circ} \mathrm{C}$. 


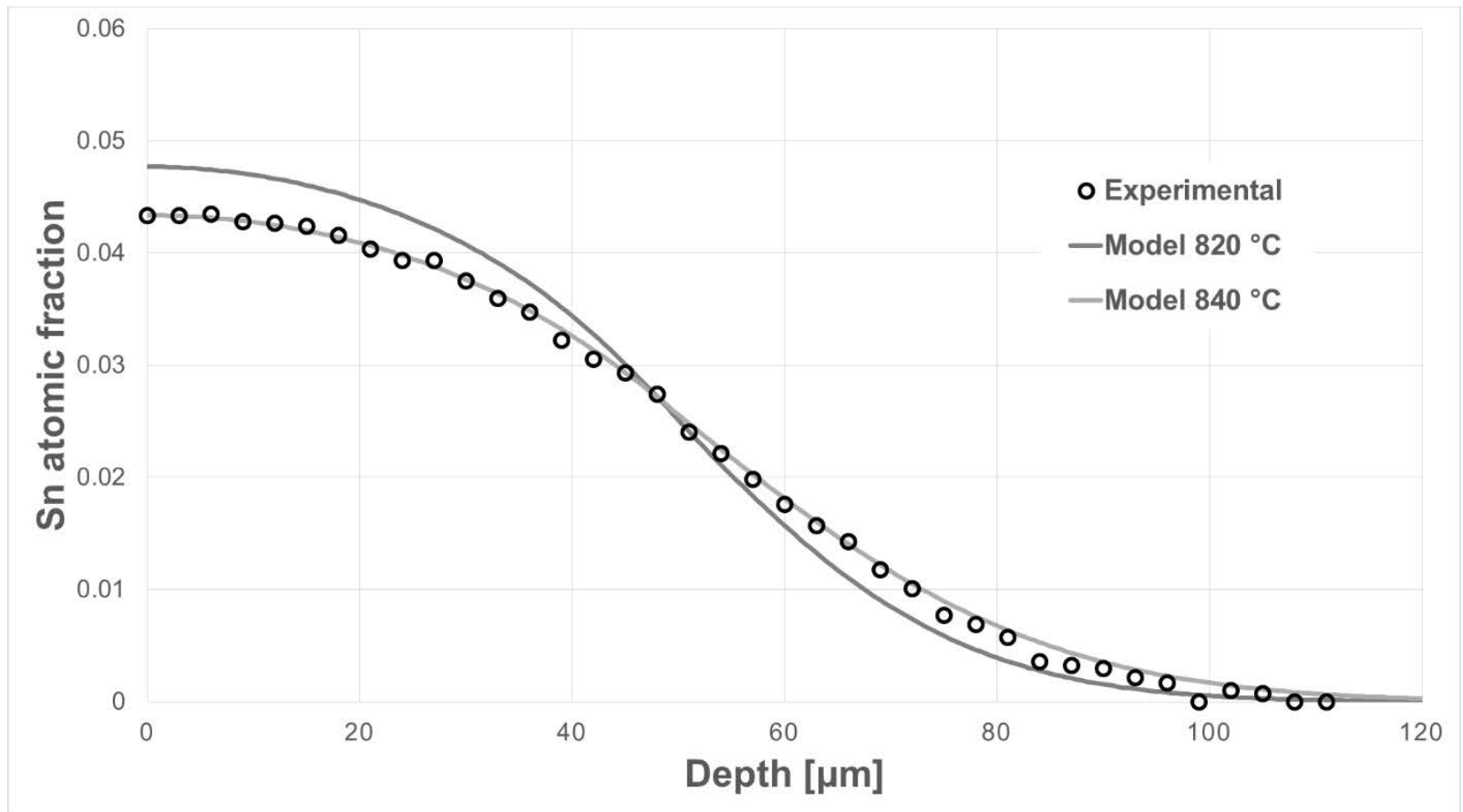

Figure 3-30 - Experimental data (circles) and model simulations (lines) of composition profile of sample \#2 heat treated at $820^{\circ} \mathrm{C}$ for $70 \mathrm{~min}$.

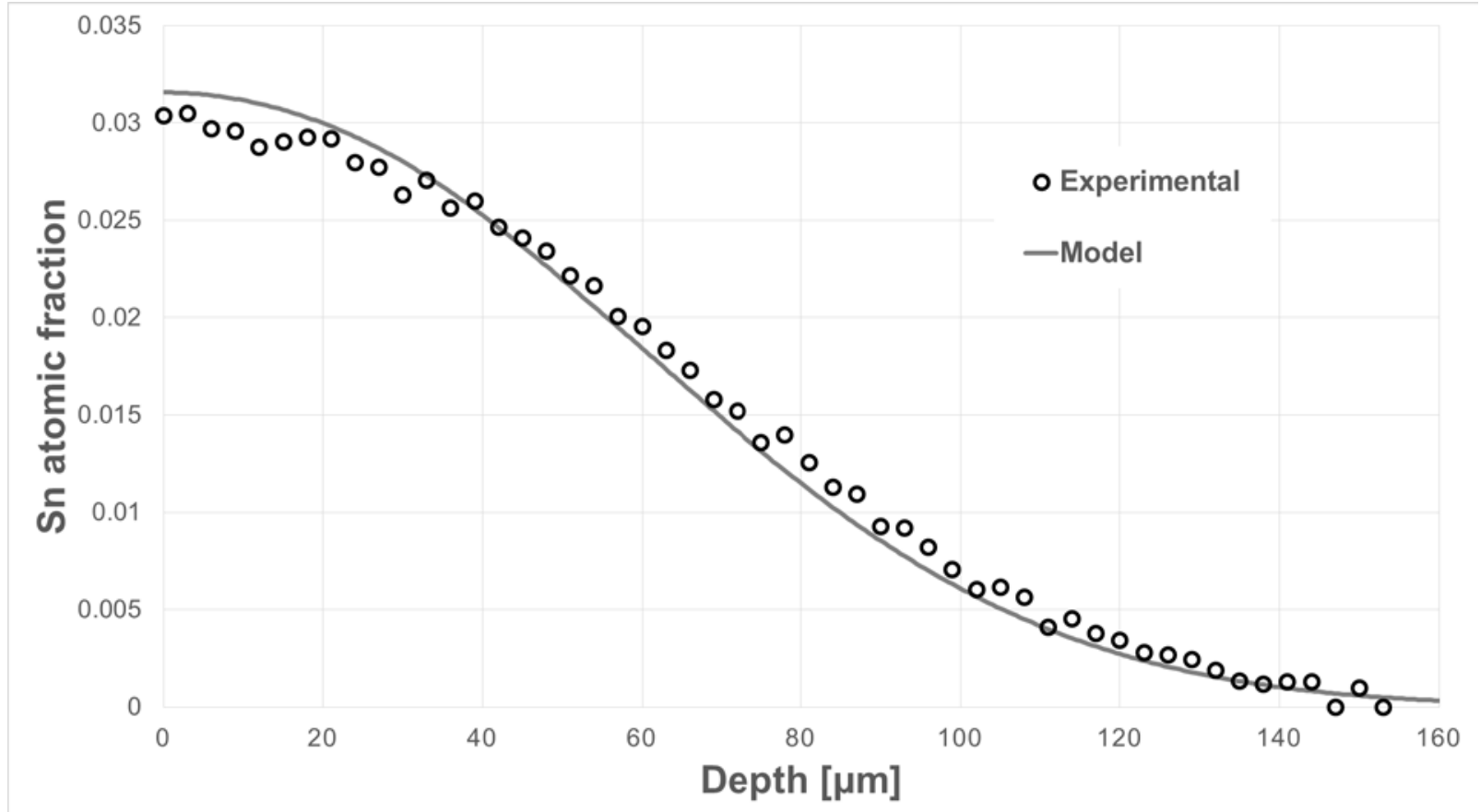

Figure 3-31 - Experimental data (circles) and model simulation (line) of composition profile of sample \#3 heat treated at $870{ }^{\circ} \mathrm{C}$ for $80 \mathrm{~min}$. Simulation temperature $=880^{\circ} \mathrm{C}$. 
On Sample \#4, the amount of Sn calculated from the experimental data resulted in an equivalent thickness of $2.71 \mu \mathrm{m}$, which is in the middle of the expected range of equivalent thickness. Just as observed on previous samples, the best fit of the simulated curve was obtained at a temperature higher than that of the furnace, which in this case is $820^{\circ} \mathrm{C}$. Figure $3-32$ shows the experimental and simulation composition profiles. The sudden jump in the composition profile observed for the first experimental data point is most likely a sample edge effect during EDS measurement.

The amount of solute calculated from the experimental data of Sample \#5 was also within the range expected, with an equivalent thickness of $2.60 \mu \mathrm{m}$. At this heat treatment temperature $\left(890^{\circ} \mathrm{C}\right)$, the experimental data showed an almost linear composition decay with depth, which was not seen in the simulation, see Figure 3-33. This means that there is considerable discrepancy between the predicted and actual composition close to the surface, with underestimations from the prediction. The difference was considerably reduced from a depth of $20 \mu \mathrm{m}$ inward, with slight overestimations from the prediction. There is most likely a change in the diffusion behavior at this temperature range, which would cause the diffusion coefficient to deviate from the Arrhenius relation that was used. Arita et al. [45] witnessed a similar phenomenon during the diffusion experiments of $\mathrm{Cu}$ and $\mathrm{Sn}$ at the $\gamma$ phase. The intrinsic diffusion coefficient of both $\mathrm{Cu}$ and $\mathrm{Sn}$ had a slope change on each of the Arrhenius plot, which took place at around $626^{\circ} \mathrm{C}$ on $\mathrm{Sn}$ and $676^{\circ} \mathrm{C}$ on $\mathrm{Cu}$, but no explanation was offered. Dohie [46] also observed a deviation of the Arrhenius relation at higher temperatures when analyzing the diffusion of zinc and iron. It was suggested that the behavior is caused by the formation of divacancies. 


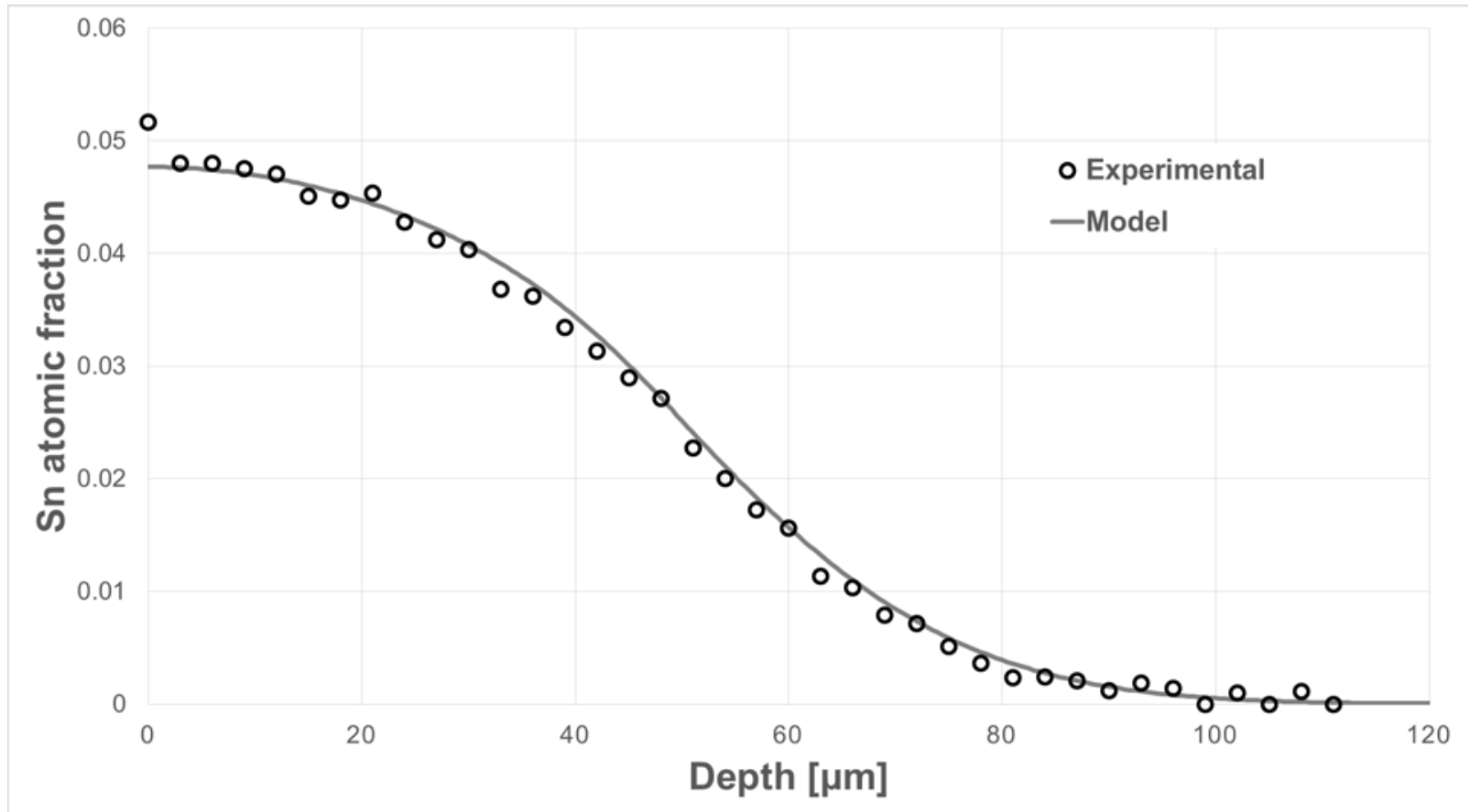

Figure 3-32 - Experimental data (circles) and model simulation (line) of composition profile of sample \#4 heat treated at $800^{\circ} \mathrm{C}$ for $70 \mathrm{~min}$. Simulation temperature $=820^{\circ} \mathrm{C}$.

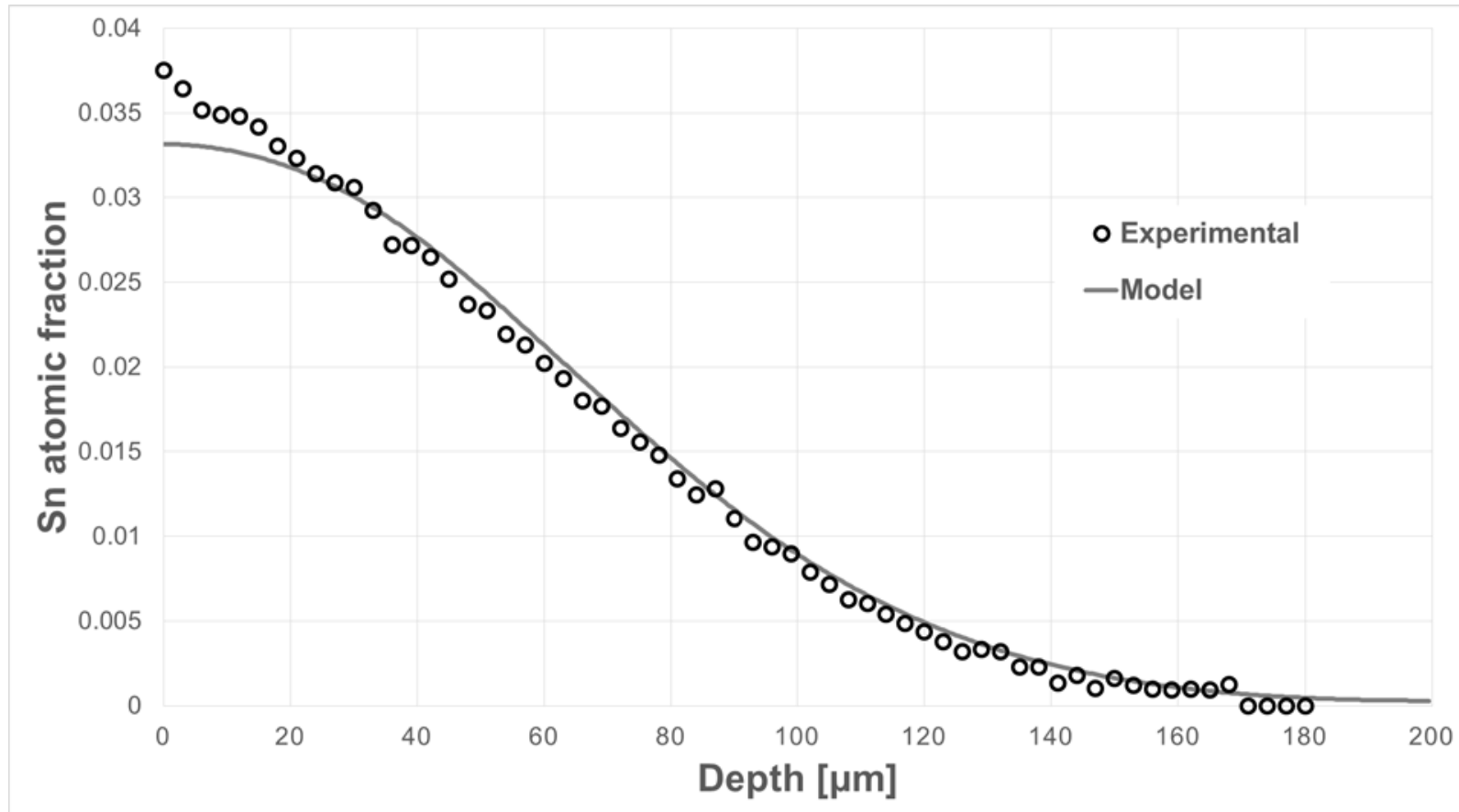

Figure 3-33 - Experimental data (circles) and model simulation (line) of composition profile of sample \#5 heat treated at $890^{\circ} \mathrm{C}$ for $80 \mathrm{~min}$. Simulation temperature $=900{ }^{\circ} \mathrm{C}$. 
For Sample \#6, simulation was done prior to the heat treatment. To reduce the uncertainty introduced by determining deposition thickness through weight change, this sample was slightly larger than the previous ones, with dimensions of $10 \mathrm{~mm}$ x $10 \mathrm{~mm} \times 10 \mathrm{~mm}$, and a more precise weight scale was utilized.

Simulation was performed at a temperature that was $10^{\circ} \mathrm{C}$ higher than that of the furnace, as an offset was observed on previous experiments. The simulation result is presented as a range, rather than a precise line, given that the deposition thickness, although more precise, is still a source of error. The heat treatment time was set as the time necessary for the complete solidification of the greater thickness estimated, represented by the upper limit of the resultant range. Conversely, the lower limit of the range represented the composition profile for a specimen with the smaller estimated thickness but subjected to the same diffusion time. Figure 3-34 shows the result of the simulation, which is the gray colored area, and the experimental composition profile obtained, which are the circles. 


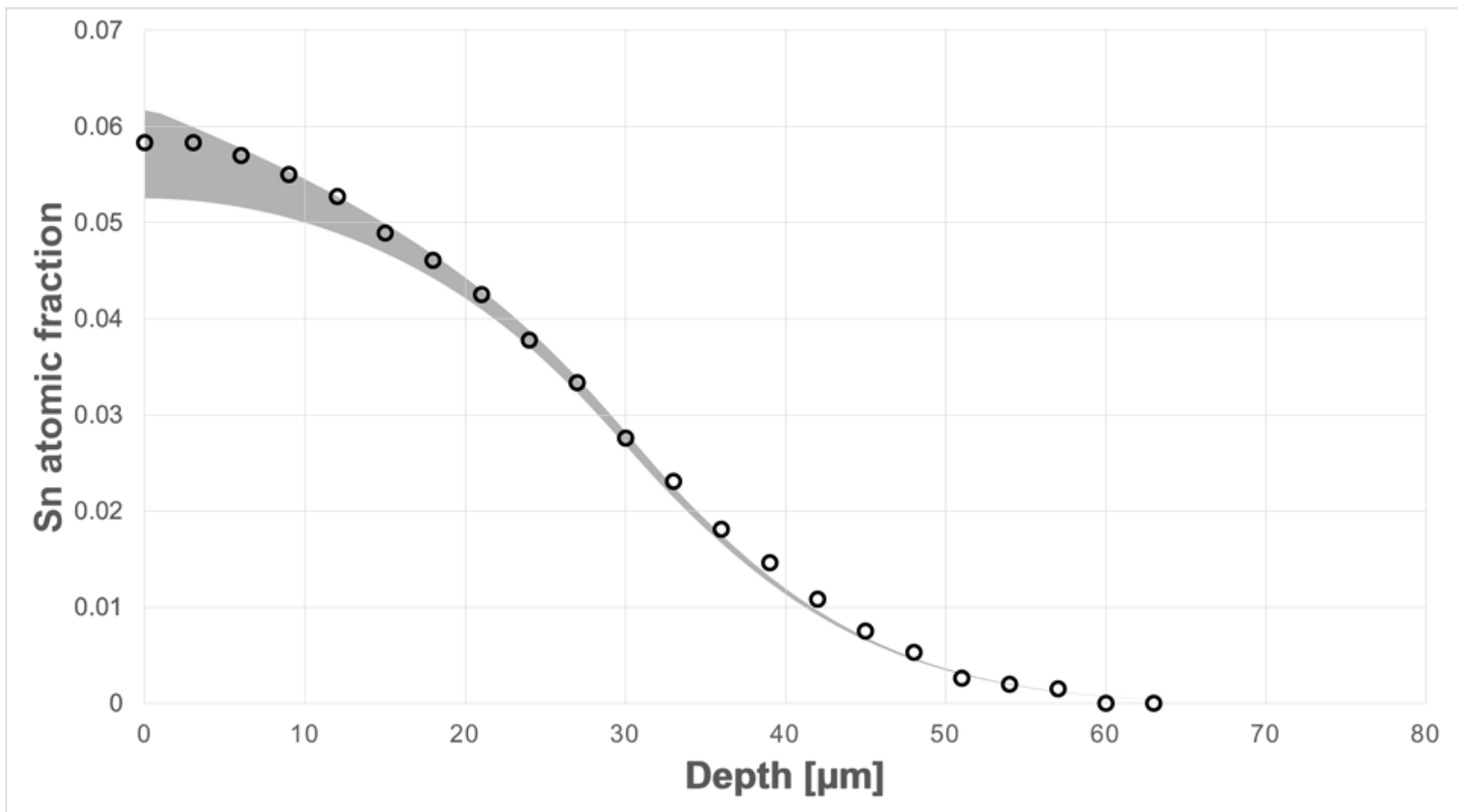

Figure 3-34 - Possible range of composition profile estimated by model (gray area) and experimental composition profile obtained from specimen heat treated at $830^{\circ} \mathrm{C}$ for 20 minutes. Simulation temperature $=840^{\circ} \mathrm{C}$. 


\subsection{Sn-rich Craters}

As mentioned in Chapter 2, the heat treatment of $\mathrm{Cu}$ coated with Sn often results in a surface that is covered with craters in which the Sn content is considerably higher than that in its surroundings. Experimentation showed that the crater formation is sporadic, and changes in size and area coverage are observed even when the experiment parameters are kept constant.

The crater topology often presents itself as two concentric circles with a higher Sn content in the inner circle. A lower concentration of Sn is found in the outer circle; however, it is still superior to that found on the unaffected regions of the specimen. At the edge of the outer circle an abrupt drop in the concentration of Sn occurs, reaching a homogeneous level throughout the unaffected surface. On some specimens, only the inner circle is present.

Figure 3-35 shows a surface optical image and below the image, a cross section BSE image, taken from the location indicated, of a sample heat treated at $810^{\circ} \mathrm{C}$ for 30 minutes. To assist with the visualization, the backscattered image is vertically stretched, thus increasing the size of the features on the vertical axis seven-fold, whereas the horizontal axis is not changed. It can be seen that the surface of the specimen unaffected by the craters is flat, in contrast to the rough profile seen on the craters. With this surface as reference, the area around the crater, which is golden on the optical image, is on a higher plateau, followed by a depression where the dark crater is located. The change in the topography suggests that, during the heat treatment, something caused the liquid phase to concentrate in some regions, thus increasing the amount of solid dissolution through diffusion. During the fourth stage, some regions had basins that contained more liquid, which created disparity on the time necessary for solidification. This way, the unaffected surfaces would reach and continue on with the fifth stage, thus reducing the surface composition, while craters still contained the liquid phase. The borders were most likely resultant of the spillage of the excess 
liquid present in the craters. At the end of the heat treatment process, these craters which were full of liquid would undergo a series of eutectic and eutectoid phase transformations. The different densities of the phases formed led to the formation of an uneven surface.

If the assumption that the topography of the craters is formed during cooling is correct, then underneath these craters the surface should be fairly even. Consequently, the composition profile of two close locations of the same crater should share the same shape when the "base" of the crater is reached, even if each chosen position has a different height. Figure 3-36 shows the BSE image of a section of the rightmost crater seen in Figure 3-35. This time, the original aspect ratio of the image is retained. Below the image is the composition profile taken from a straight line from top to bottom of the locations identified as A and B, as well as the composition profile obtained by simulation of the diffusion of a semi-infinite liquid layer, similar to what was done with Sample \#1 in a previous section of this chapter. All of the profiles are aligned according to the indicated dashed line, chosen according to the deepest eutectic formation. As predicted, both profiles align with each other, thus indicating a common even base, and with the simulated profile, indicating that there is no unusual diffusion that created these craters.

As was discussed in the previous chapter, the $\varepsilon$ and $\eta$ intermetallic phases form heterogeneously and very quickly during heat treatment. The formation of these phases is most likely unavoidable. However, their heterogeneity can be reduced by allowing enough time for these phases to form properly. A set of samples coated with approximately $3.5 \mu \mathrm{m}$ of Sn were used to test the intermetallic influence. Some of them were heat treated at $250^{\circ} \mathrm{C}$ to allow for the formation of the $\eta$-phase, while others were heat treated at $450^{\circ} \mathrm{C}$, for the exclusive formation of the $\varepsilon$-phase. 


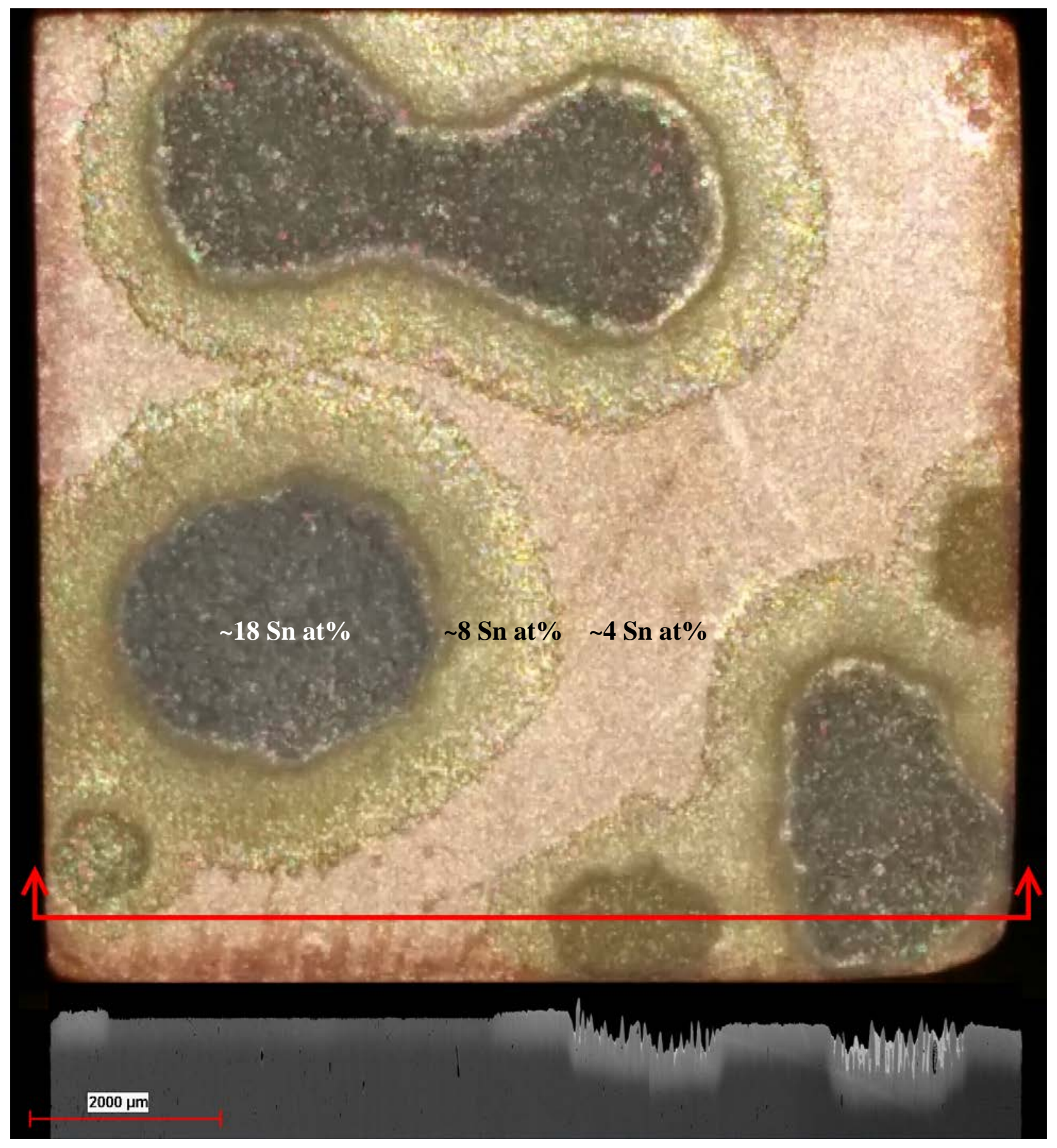

Figure 3-35 - Top: optical image of surface of specimen heat treated at $810{ }^{\circ} \mathrm{C}$ for 30min; average surface composition of each region as shown. Bottom: BSE image of cross-section stretched on vertical axis. Location of cross-section indicated by line on optical image 

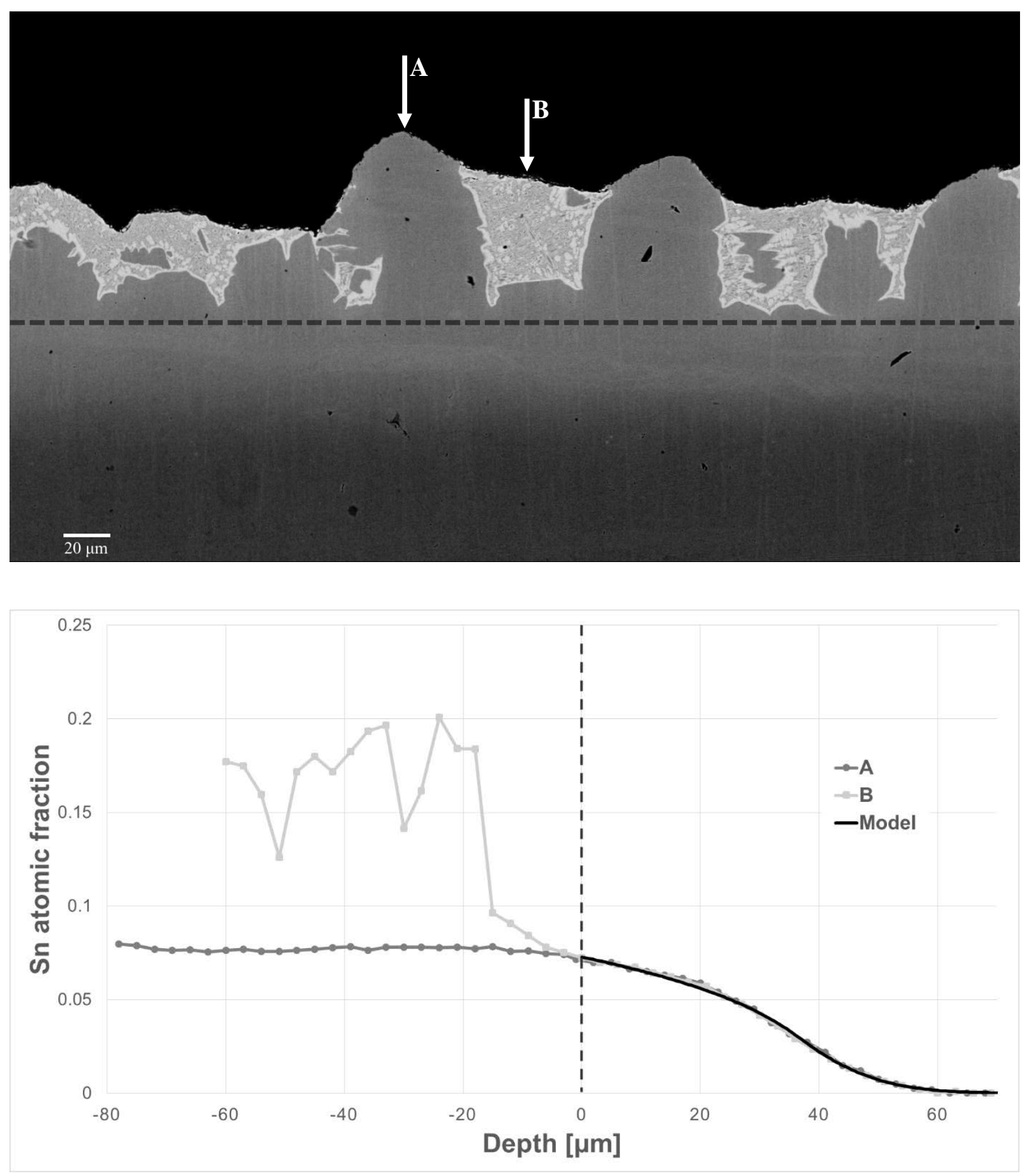

Figure 3-36 - Top: BSE cross-section image of tin-rich crater. Bottom: Composition profiles obtained from locations indicated on BSE image and simulated by model. 


\subsubsection{Heat Treatment at $450^{\circ} \mathrm{C}$}

At $450^{\circ} \mathrm{C}, \varepsilon$ is the first phase to form and $\eta$ should not be found. The formation of any other solid phase at this temperature would have to transform into either the $\varepsilon$ or $\alpha$ phase to remain stable after cooling. Therefore, the presence of $\eta$-phase after heat treatment at this temperature is explained only by the cooling of excess liquid.

When heat treated for 15 minutes, the specimen displayed circular features on its surface which resemble the Sn-rich craters, but are concave rather than convex, as seen in Figure 3-37a and Figure 3-37b. EDS analysis showed that the protrusions are composed of 100 Sn at\%. After analyzing the cross-section, a clear delimitation between $\mathrm{Cu}$ and $\varepsilon$-phase can be seen, see Figure 3-37c and Figure 3-37d, with virtually no diffusion of Sn into $\mathrm{Cu}$ as found by the EDS analysis, see Figure 3-37e. The cross section of the protrusion can be seen in Figure 3-38a, which reveals that although it seems to be distended from the surface, it is indeed cratering into the material as well. Once again, the presence of the $\eta$-phase and (Sn) indicates that liquid was concentrated in this region.

A second sample was heat treated for the same duration of time, which resulted in a reduced number of protrusions on the surface. This sample was then heat treated at $830^{\circ} \mathrm{C}$ for 23 minutes. This duration of time was predicted by the model as the time necessary for complete solidification, assuming a step composition profile between the $\varepsilon$-phase layer and pure $\mathrm{Cu}$ substrate, that is, no Sn diffusion into the bulk Cu prior to the high temperature heat treatment. Figure 3-38b shows the composition range of the prediction due to the uncertainty of actual deposited Sn (gray area) superimposed with the composition profile of the actual composition obtained from a cross section analysis of the sample. Figure 3-39a and Figure 3-39b are the optical images of the surface of the specimen after the first and second heat treatments. From the location of the protrusions, it is clear that they are the cause of the Sn-rich craters on the final surface. 

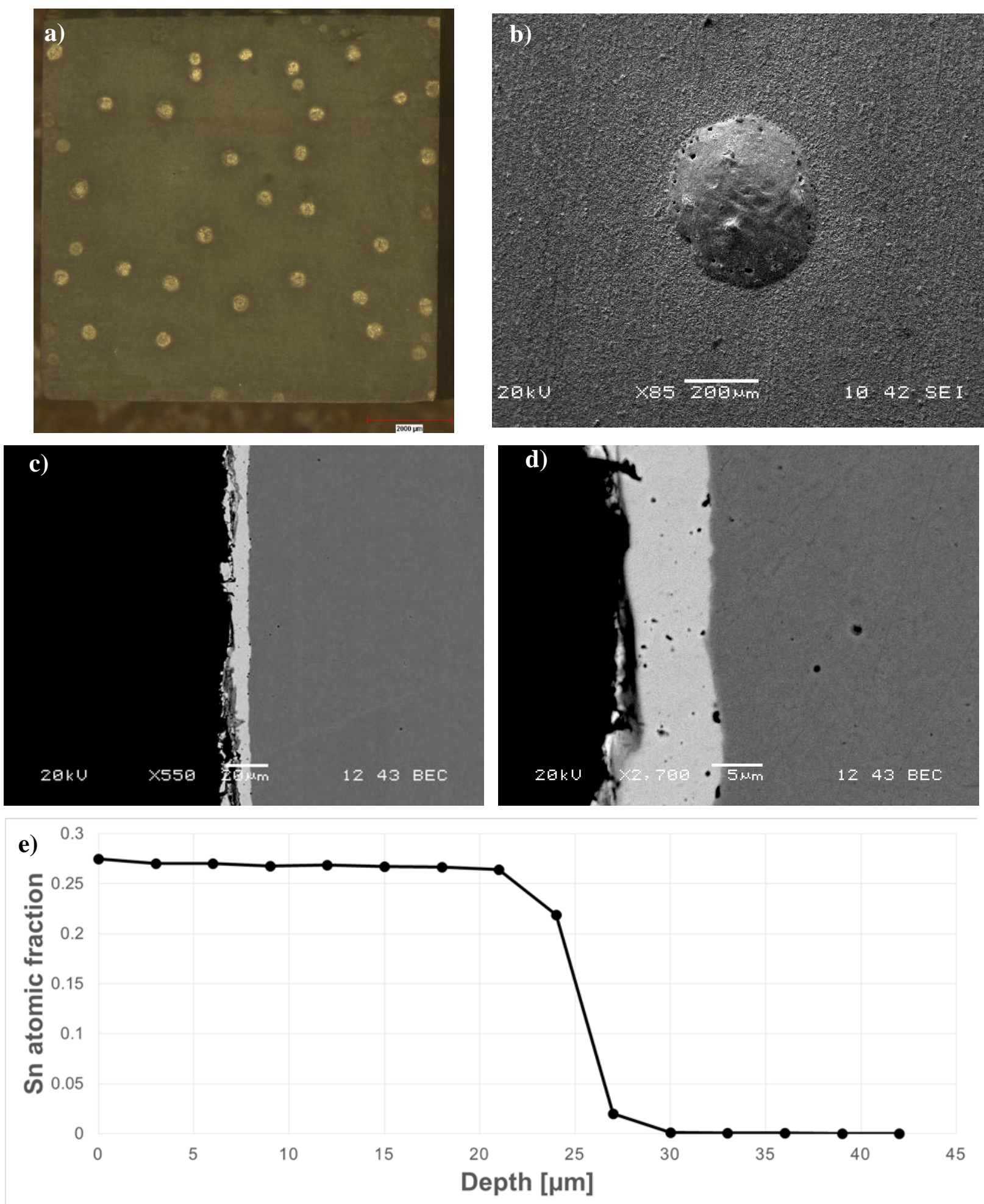

Figure 3-37 - Specimen heat treated at $450{ }^{\circ} \mathrm{C}$ for 15 min. a) optical surface image; b) SE surface image; c) BSE cross-section image; d) higher magnification BSE cross-section image; e) EDS point scan forming composition profile of location shown on $d$ ). 

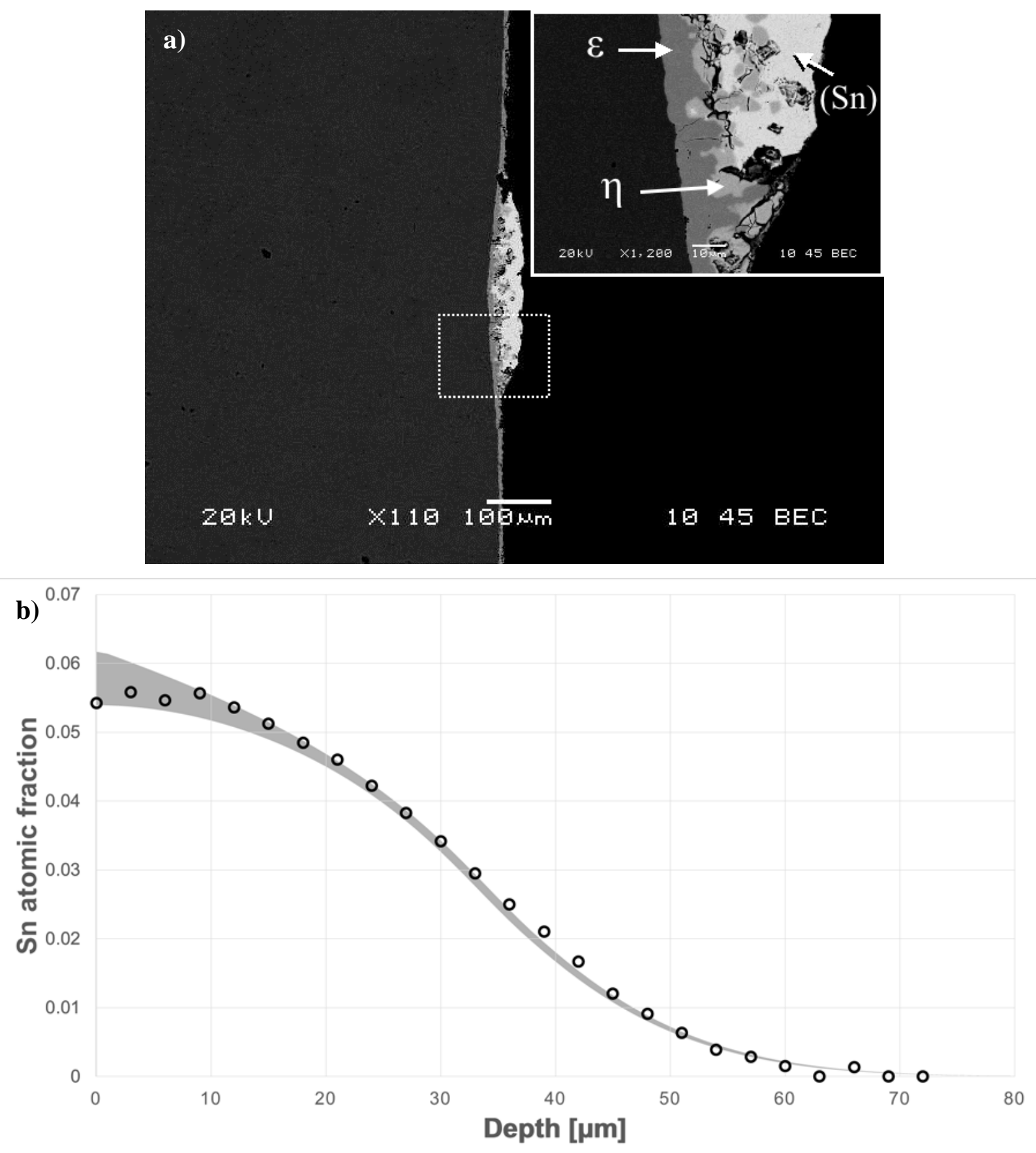

Figure 3-38 - a) cross-section image from protrusion of specimen heat treated at $450{ }^{\circ} \mathrm{C}$ for $15 \mathrm{~min} . \mathrm{b}$ ) Composition range predicted (gray area) and measured composition profile (circles) of sample heat treated at $450{ }^{\circ} \mathrm{C}$ for $15 \mathrm{~min}$ followed by $830^{\circ} \mathrm{C}$ for $23 \mathrm{~min}$. 

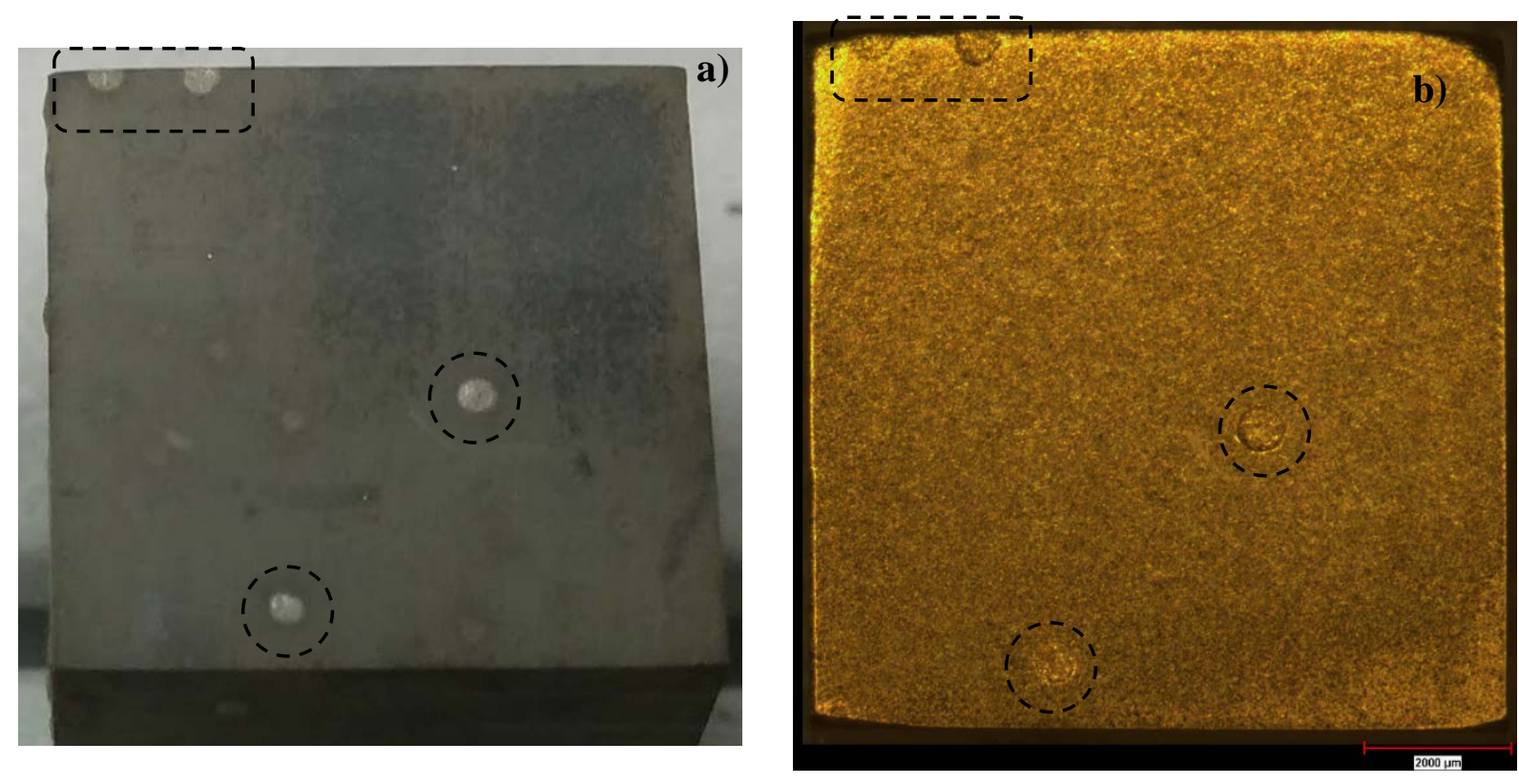

Figure 3-39 - a) specimen heat treated at $450{ }^{\circ} \mathrm{C}$ for $15 \mathrm{~min}$. b) same specimen as in " $a$ " heat treated at $830^{\circ} \mathrm{C}$ for $23 \mathrm{~min}$. 
Other samples were tested at increased heat treatment time (30 minutes) at $450^{\circ} \mathrm{C}$, but surface protrusions could not be completely eliminated.

\subsubsection{Heat Treatment at $250^{\circ} \mathrm{C}$}

During cooling after carrying out heat treatment at $250^{\circ} \mathrm{C}$, any excess liquid phase would result on the formation of (Sn) and $\eta$ at a ratio of approximately 20:1, in accordance with the lever rule. Any other solid phases would have been formed as a result of diffusion during the heat treatment.

Just like on the specimens heat treated at higher temperatures, at $250^{\circ} \mathrm{C}$ the resultant surface displays protrusions composed of Sn, see Figure 3-40a and Figure 3-40b. The hard and brittle nature of the $\eta$-phase interferes with the cross-section analysis, causing the ductile Cu substrate to deform during polishing, see Figure 3-40c. $\eta$ and (Sn) are the only phases found; like before, no significant diffusion of Sn into the Cu substrate can be detected. A cross-section analysis of the protrusions shows a high volume of (Sn), as expected from the cooling of the excess liquid phase, see Figure 3-40d. At the interface of the protrusions and Cu substrate, a thin heterogeneous layer of the $\eta$-phase can be seen; cratering is not evident as it is on the specimens that were heat treated at a higher temperature.

Increasing the heat treatment time to 30 minutes led to some cases where the protrusions were eliminated on the surface and others where they were still present. However, unlike the previous samples, a second heat treatment at a higher temperature $\left(+800^{\circ} \mathrm{C}\right)$ resulted in surfaces that are devoid of Sn-rich craters in both cases, see Figure 3-41. 

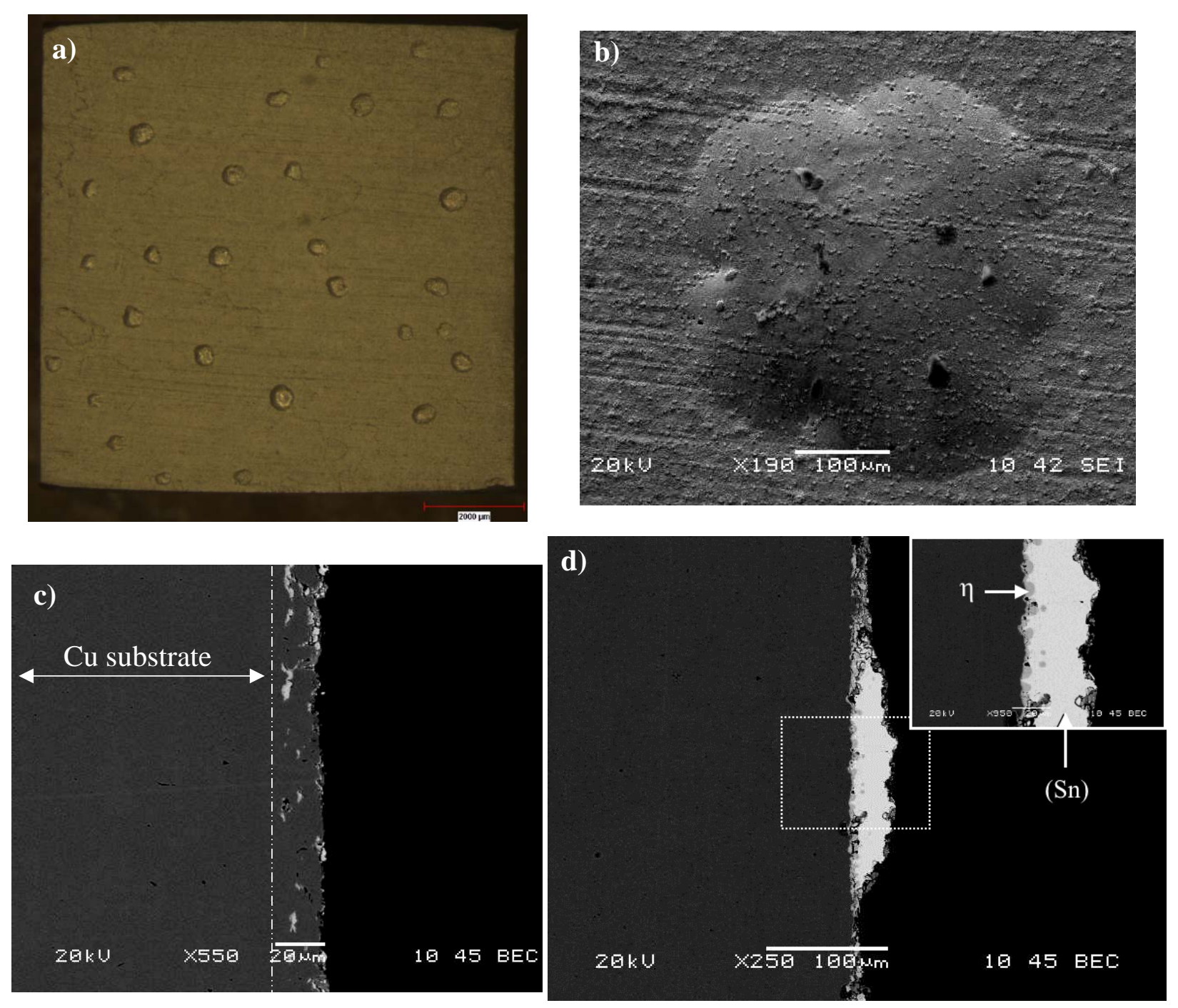

Figure 3-40 - a) surface optical image of sample heat treated at $250{ }^{\circ} \mathrm{C}$; b) surface SEM image of one of the protrusions from sample on a); c) cross-section SEM image showing copper covering surface layer;

d) cross-section BSE image of protrusion. 

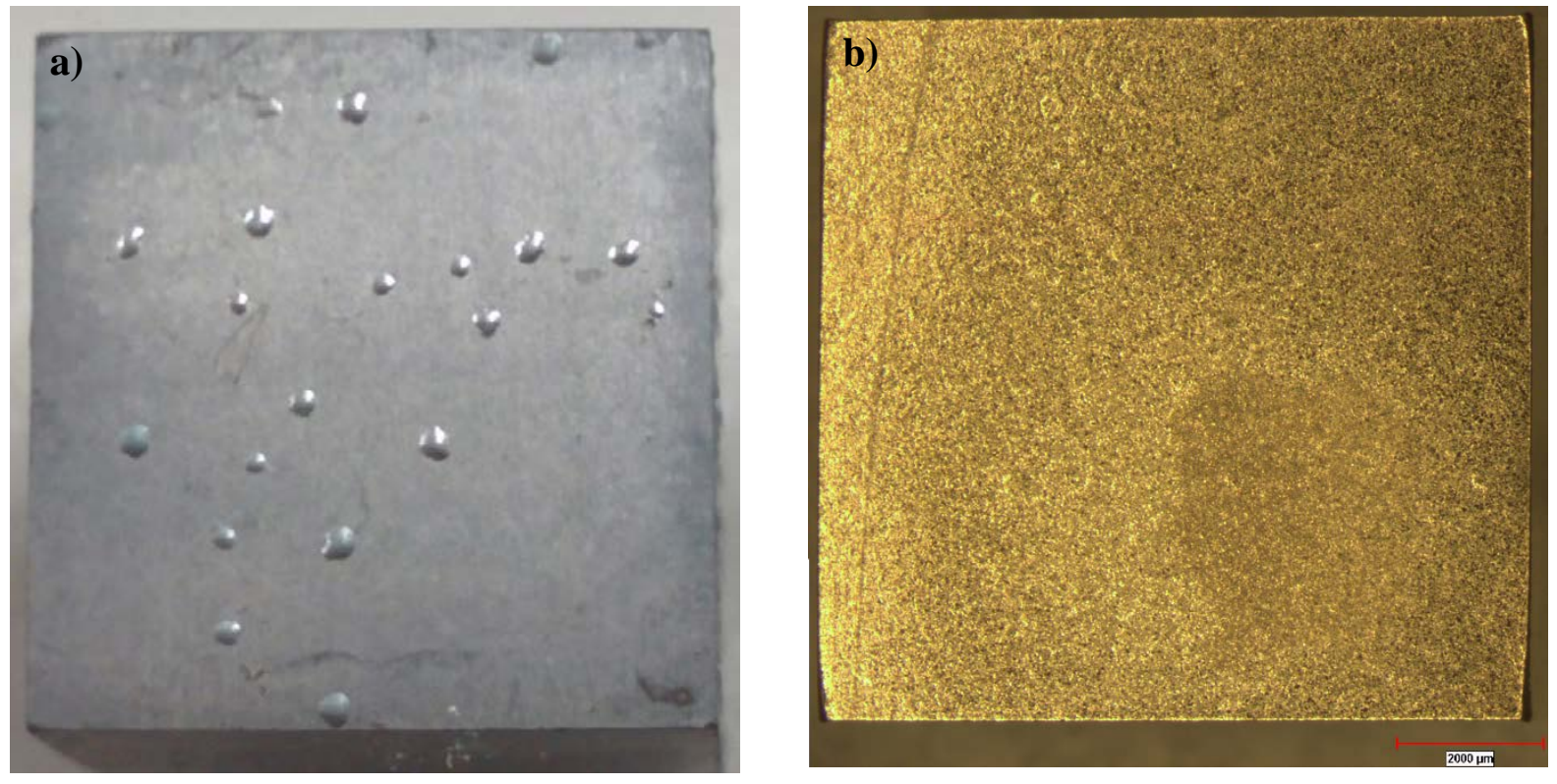

Figure 3-41 - a) specimen heat treated at $25^{\circ} \mathrm{C}$ for 30min; b) same specimen after heat treatment at $830{ }^{\circ} \mathrm{C}$ for $20 \mathrm{~min}$. 


\subsubsection{Proposed Mechanism of Crater Formation}

Taking into consideration the results, it is most likely that the Sn-rich craters observed on the heat treated samples were caused by a wetting problem. When Sn reached its melting temperature, the liquid phase formed bubbles on the specimen surface, similar to reports in the literature on the poor wettability performance of $\mathrm{Sn}-\mathrm{Cu}$ solders below $300^{\circ} \mathrm{C}$ [47]. As the temperature further increased towards the targeted temperature, the $\mathrm{Cu}$ solubility in the liquid phase quickly increased, thus leading to excessive dissolution of the Cu substrate where bubbles were present, and creating craters. Upon reaching the working temperature, diffusion proceeded normally, however, the craters held an increased amount of liquid. As the unaffected surface reached complete solidification, the craters were still covered with liquid. At the end of the heat treatment, the liquid in the craters underwent a series of phase transformations which resulted in undesirable phases and topography.

The formation of intermetallic phases at the solid/liquid interface created a protective barrier due to the low diffusion coefficient of the said phases [48], thus allowing temperature and wetting to increase. The formation of an intermetallic layer by itself may also had contributed to an increased surface energy, thus increasing wetting. This would explain the lack of protrusions on some of the samples, after heat treatment at $250^{\circ} \mathrm{C}$ was held long enough for a complete intermetallic layer to form. At $450^{\circ} \mathrm{C}$, the $\mathrm{Cu}$ solubility in Sn was considerably higher, thus allowing the formation of craters before an intermetallic layer was established and leading to the formation of protruding craters found after the heat treatment. By heat treating a specimen with an $\eta$ intermetallic layer, working temperature $\left(+800^{\circ} \mathrm{C}\right)$ could be reached with an even surface which will then simultaneously achieve complete solidification without craters. 


\subsection{Hardness Measurement}

A nanomechanical test instrument, TI 750 Ubi L (Hysitron) was used to assess the changes in surface hardness with increased Sn content. Heat treated samples were mounted with epoxy thus exposing one of its surfaces, which was checked by using EDS on a SEM to determine its average composition. For that, at least ten points were taken from areas close to the corners and center of the exposed surface. Hardness testing was performed with a Berkovich indenter, in which the initial contact force was set at $5 \mu \mathrm{N}$ and peak load at $1000 \mu \mathrm{N}$, held constant for 2 seconds. Maximum displacement observed on the tested samples was in the order of $100 \mathrm{~nm}$. Figure 3-42 shows a typical load-displacement curve obtained during the nanoindentation hardness testing with data points taken at intervals of $0.005 \mathrm{~s}$. By analyzing the hardness of the different surface compositions, a clear trend is established, in which an increase in the Sn content leads to an increase in hardness, as shown in Figure 3-43. This result confirms that alloying $\mathrm{Cu}$ with $\mathrm{Sn}$ produces better mechanical properties compared to pure $\mathrm{Cu}$ metal, and the composition profile modeling work done in this thesis would help in producing bronze coatings with the desired composition. 


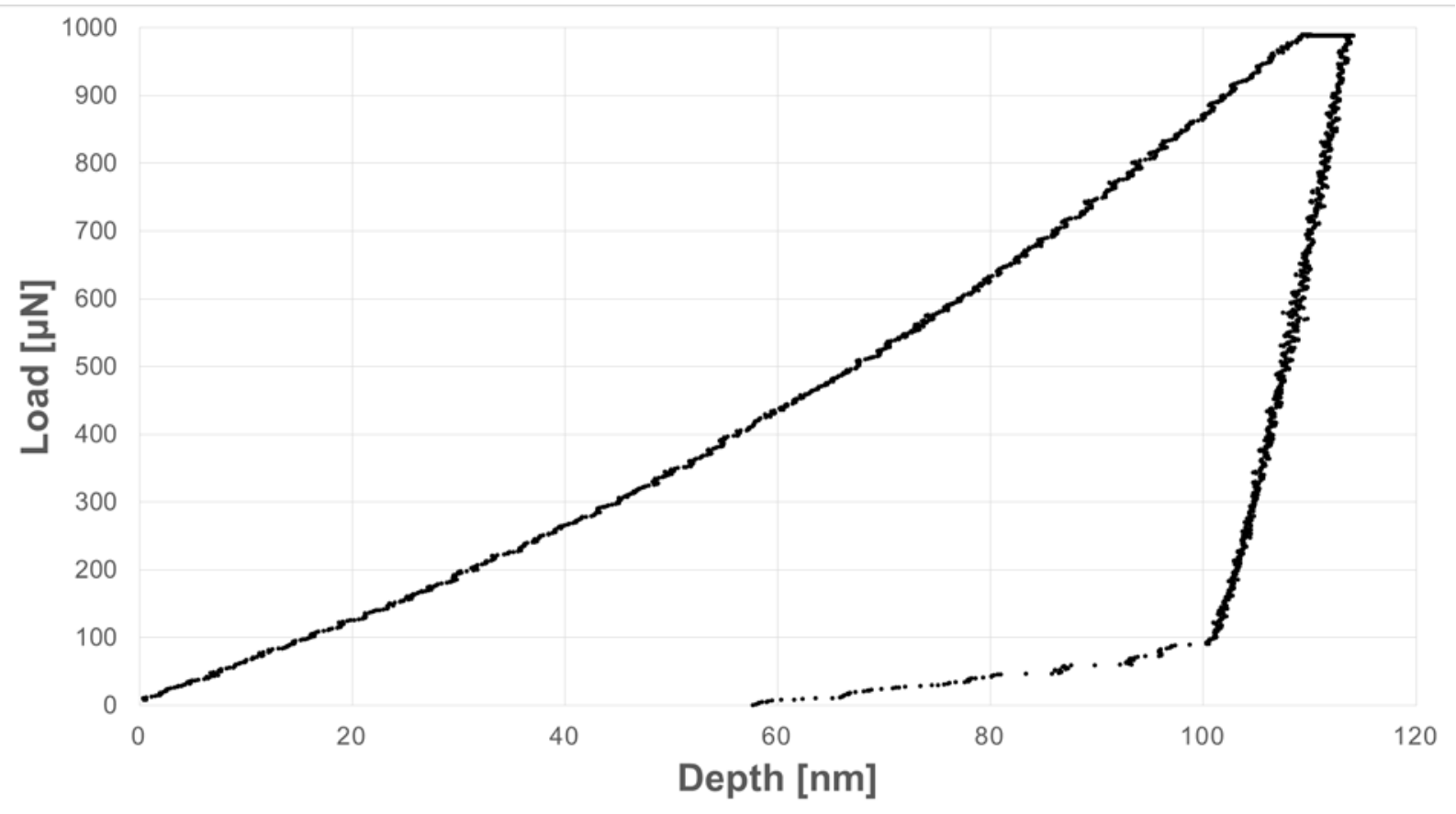

Figure 3-42 - Typical load-displacement curve obtained during nanoindentation testing.

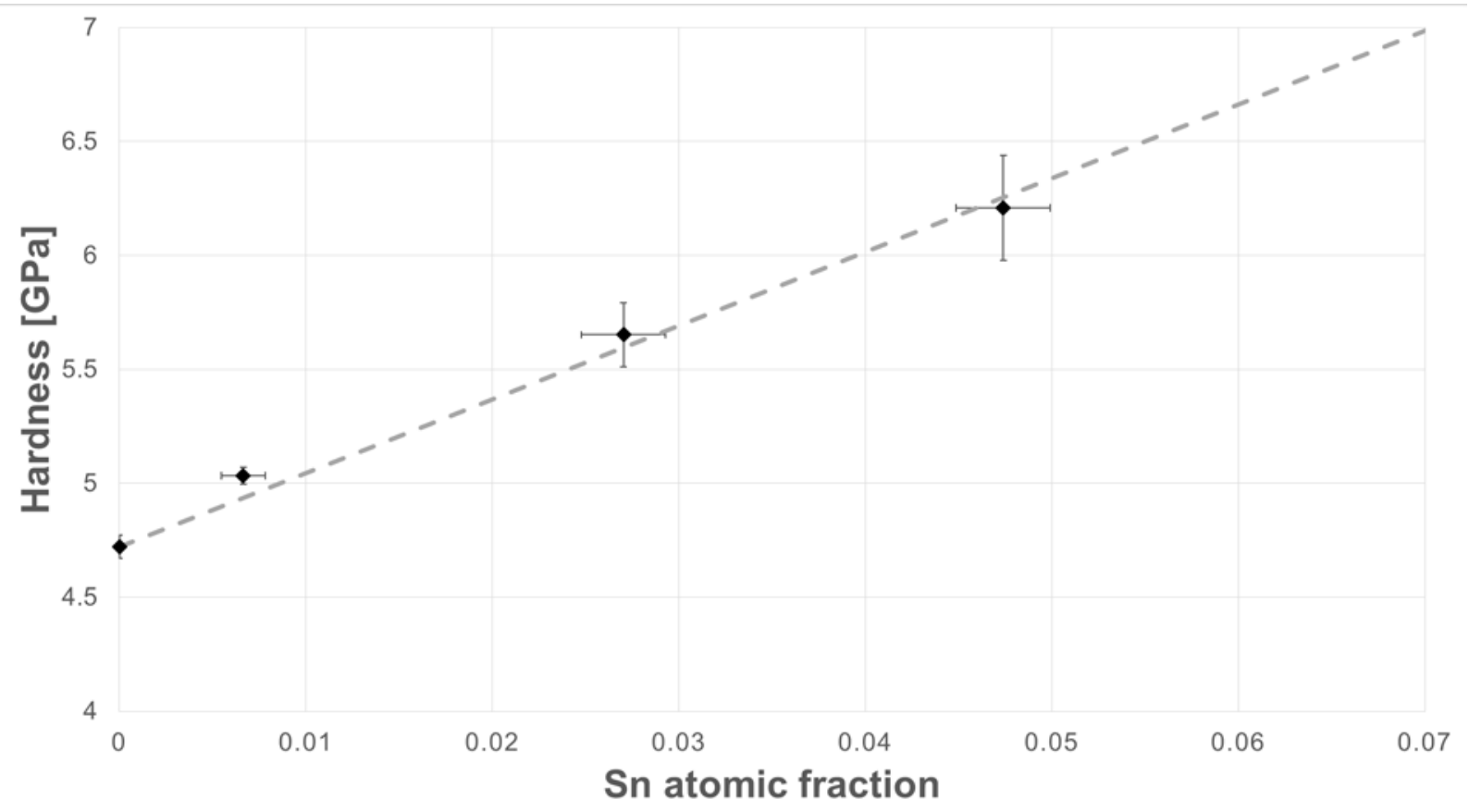

Figure 3-43 - Nanoindentation hardness test results for different surface compositions. 


\section{Chapter 4 Conclusions and Recommendations for Future Work}

The objective of this research work is to elucidate the physical processes that occur during the diffusion heat treatment of $\mathrm{Cu}$ and $\mathrm{Sn}$ for the formation of bronze and, to use a numerical model to predict the resultant composition profile after diffusion heat treatment. The major findings in the research are summarized below:

1. The Cu-Sn phase diagram has been analyzed to explain the behavior during heat treatment, which can be divided into five stages: the first stage has little contribution to the overall process; the second and third stages have the same behavior but a temperature gradient is present on the second, therefore with a high enough heating rate, the second stage can be disregarded and replaced by the third; the fourth stage, in which solidification occurs, is the most crucial due to a decrease in diffusion rate, making it the longest; in the fifth stage, only solid state diffusion occurs and usually heat treatment is aimed to end at the very beginning of this stage to attain maximum surface concentration of Sn.

2. A numerical model was built based on the above behavior. To address the Stephan condition, time and spatial stepping are altered accordingly at the interphase between liquid and solid, leading to improved accuracy while avoiding any excessive increase in computational cost.

3. Simulations showed that, in the temperature range considered, solidification is slower at higher temperatures due to decreased solubility which leads to a smaller concentration gradient, reducing the extent of diffusion. However, under the same concentration gradient, which occurs after solidification, higher temperatures exhibit higher diffusion rates due to an increased diffusion coefficient.

4. Temperature also influences the overall shape of the composition-depth curve; at higher temperatures, $\mathrm{Sn}$ is distributed deeper into the bulk of the sample, creating a profile that 
quickly decreases in Sn composition close to the surface but then retains a higher composition deeper into the bulk. Samples heat treated at lower temperatures have a higher Sn concentration close to the surface, which is compensated later, with a lower concentration deeper into the bulk.

5. Experimental verification showed a very good correlation with the simulation predictions. The processing parameters, such as temperature, time and deposition thickness, proved to be crucial in determining the correct composition profile. At the higher temperature range tested, the model shows some errors close to the surface, which could be caused by a transition to a different diffusion behavior.

6. Sn-rich craters appear to be caused by the agglomeration of the liquid phase in some discrete regions. These regions require more $\mathrm{Cu}$ dissolution to reach liquid saturation, thus leading to heterogeneous substrate dissolution that generates the craters.

7. Introducing a barrier of intermetallic phase prevents diffusion while temperature is increased to the point where wetting is enough to allow the liquid phase to uniformly spread. This method is tested at temperatures of $250^{\circ} \mathrm{C}$ and $450^{\circ} \mathrm{C}$, but only successful at $250^{\circ} \mathrm{C}$, because at the higher temperature Cu solubility on Sn is enough to create craters. In theory, this method is feasible below the melting temperature of $\mathrm{Sn}$, as the formation of both intermetallics are reported, however, the time required to do so would increase considerably.

\subsection{Suggestions for future work}

In this work, a model is used to simulate the heat treatment process of $\mathrm{Cu}-\mathrm{Sn}$ which is formulated based on phase diagram observations. For future work, the following is suggested.

As mentioned in Chapter 3, the simulation of the second stage of heat treatment does not consider the formation of other phases found below $800^{\circ} \mathrm{C}$. The inclusion of other phases would 
increase the temperature range at which the model can be applied. However, the addition of these phases is not an easy task, since complex phase transformations are involved, such as orderdisorder and intermetallic formation. Furthermore, precise information of the behavior of the diffusion coefficient of each phase involved would be required. Optimization of the intermetallic formation to prevent the development of Sn-rich craters can also be investigated to identify the ideal combinations of temperature and time. 


\section{References}

[1] R. C. Tucker and P. S. T. Inc, ASM Handbook - Surface Engineering. 1994.

[2] D. J. R., Copper and Copper Alloys. 2001.

[3] B. Subramanian, S. Mohan, and S. Jayakrishnan, "Structural, microstructural and corrosion properties of brush plated copper-tin alloy coatings,” Surf. Coatings Technol., vol. 201, no. 3-4, pp. 1145-1151, 2006.

[4] Y. Sürme, A. A. Gürten, E. Bayol, and E. Ersoy, "Systematic corrosion investigation of various Cu-Sn alloys electrodeposited on mild steel in acidic solution: Dependence of alloy composition,” J. Alloys Compd., vol. 485, no. 1-2, pp. 98-103, 2009.

[5] S. Fürtauer, D. Li, D. Cupid, and H. Flandorfer, “The Cu-Sn phase diagram, Part I: New experimental results,” Intermetallics, vol. 34, pp. 142-147, 2013.

[6] D. Li, P. Franke, S. Fürtauer, D. Cupid, and H. Flandorfer, “The Cu-Sn phase diagram part II: New thermodynamic assessment,” Intermetallics, vol. 34, pp. 148-158, 2013.

[7] C. Hasse and F. Pawlek, “Zur Kenntnis der Kupfer-Zinnlegierungen,” Z. Allg. Mikrobiol., vol. 28, pp. 73-80, 1936.

[8] N. Okamoto, Y. Fujii, H. Kurihara, and K. Kondo, "Effects of Microstructure of Deposited Sn Films and Orientation Index of $\mathrm{Cu}$ Foils on Sn Whisker Formation Using Substitutionally-Deposited Sn Films,” Mater. Trans., vol. 50, no. 11, p. 2570, 2009.

[9] W. ming TANG, A. qiang HE, Q. LIU, and D. G. IVEY, "Solid state interfacial reactions in electrodeposited Cu/Sn couples,” Trans. Nonferrous Met. Soc. China (English Ed., vol. 20, no. 1, pp. 90-96, 2010. 
[10] X. Ma, F. Wang, Y. Qian, and F. Yoshida, "Development of Cu-Sn intermetallic compound at Pb-free solder/Cu joint interface,” Mater. Lett., vol. 57, no. 22-23, pp. 3361-3365, 2003.

[11] B. Chao, S. H. Chae, X. Zhang, K. H. Lu, J. Im, and P. S. Ho, "Investigation of diffusion and electromigration parameters for $\mathrm{Cu}-\mathrm{Sn}$ intermetallic compounds in $\mathrm{Pb}$-free solders using simulated annealing,” Acta Mater., vol. 55, no. 8, pp. 2805-2814, 2007.

[12] K. Fujiwara, M. Ohtani, T. Isu, S. Nango, R. Kawanaka, and K. Shimizu, "INTERFACIAL REACTION IN BIMETALLIC Sn/Cu THIN FILMS.,” Thin Solid Films, vol. 70, no. 1. pp. 153-161, 1980.

[13] K. N. Tu, "Interdiffusion and reaction in bimetallic Cu-Sn thin films," Acta Metallurgica, vol. 21, no. 4. pp. 347-354, 1973.

[14] S. Kumar, C. A. Handwerker, and M. A. Dayananda, "Intrinsic and interdiffusion in Cu-Sn system,” J. Phase Equilibria Diffus., vol. 32, no. 4, pp. 309-319, 2011.

[15] R. a. Gagliano, G. Ghosh, and M. E. Fine, "Nucleation kinetics of Cu6Sn5 by reaction of molten tin with a copper substrate,” J. Electron. Mater., vol. 31, no. 11, pp. 1195-1202, 2002.

[16] V. Vuorinen, "INTERFACIAL REACTIONS BETWEEN SN-BASED SOLDERS AND COMMON METALLISATIONS USED IN ELECTRONICS,” Helsinki University of Technology, 2006.

[17] N. Saunders and A. Miodownik, "The Cu-Sn (Copper-Tin) System,” Bull. Alloy Phase Diagrams, vol. 11, no. 3, pp. 278-287, 1990.

[18] P. Shewmon, Difffusion in Solids, 2nd ed. The Minerals, Metals \& Materials Society, 1989. 
[19] P. Shewmon, Transformations in Metals. 1969.

[20] A. Fick, “On liquid diffusion,” J. Memb. Sci., vol. 100, no. 1, pp. 33-38, Mar. 1995.

[21] R. J. Borg and G. J. Dienes, An Introduction to Solid State Diffusion. 1988.

[22] J. Crank, The Mathematics of Diffusion, Second. Oxford University Press, 1975.

[23] L. S. Darken, "Diffusion, mobility and their interrelation through free energy in binary metallic systems,” Metall. Mater. Trans. A Phys. Metall. Mater. Sci., vol. 41, no. 3, pp. 184555, 1948.

[24] D. B. Butrymowicz, J. R. Manning, and M. E. Read, "Diffusion in Copper and Copper Alloys," Journal of Physical and Chemical Reference Data, vol. 2, no. 3. pp. 643-656, 1973.

[25] S. J. Rothman and N. L. Peterson, "Isotope Effect and Divacancies for Self-Diffusion in Copper,” Phys. Status Solidi, vol. 35, pp. 305-312, 1969.

[26] P. J. Fensham, “Self-Diffusion in Tin Crystals,” Aust. J. Chem., vol. 3, no. 1, pp. 91-104, 1950.

[27] A. Bruson and M. Gerl, “Diffusion d'impuretés dans le cuivre et l'étain liquides,” J. Phys., vol. 41, no. 6, pp. 533-542, 1980.

[28] K. Hoshino, Y. Iijima, and K.-I. Hirano, "Interdiffusion and Kirkendall effect in $\mathrm{Cu} — \mathrm{Sn}$ alloys,” Philosophical Magazine A, vol. 44, no. 4. pp. 961-972, 1981.

[29] Y. D. Gamburg and G. Zangari, Theory and Practice of Metal Electrodeposition, vol. 53, no. 9. New York, NY: Springer New York, 2011. 
[30] A. J. de Bethune, N. A. S. Loud, and C. V. King, "Standard Aqueous Electrode Potentials and Temperature Coefficients at 25ㄷ,” J. Electrochem. Soc., vol. 112, no. 4, p. 107C, 1965.

[31] D. R. GABE, "The role of hydrogen in metal electrodeposition processes," J. Appl. Electrochem., vol. 27, no. 8, pp. 908-915, 1997.

[32] J. Stefan, “Über einige Probleme der Theorie der Wärmeleitung.,” Wien. Ber., vol. 98, pp. 473-484, 1888.

[33] J. Crank, Free and moving boundary problems. 1987.

[34] J. Crank and R. S. Gupta, "A moving boundary problem arising from the diffusion of oxygen in absorbing tissue," J. Inst. Math. Appl., vol. 10, pp. 19-33, 1972.

[35] W. D. Murray and F. Landis, "Numerical and machine solutions of transient heatconduction problems involving melting or freezing Part I -- Method of analysis and sample solutions,” Trans. ASME, J. Heat Transf., vol. 81, pp. 106 - 112, 1959.

[36] J. Douglas, Jr. and T. M. Gallie, Jr., "On the Numerical Integration of a Parabolic Differential Equation Subject to a Moving Boundary Condition,” Duke Math. J., vol. 22, no. 4, pp. 557-571, Dec. 1955.

[37] H. G. Landau, "Heat conduction in a melting solid,” Q. Appl. Math., vol. 8, pp. 81-94, 1950.

[38] A. C. Fischer-Cripps, “Nanoindentation,” pp. 21-38, 2011.

[39] S. Patankar, Numerical heat transfer and fluid flow. Hemisphere Publishing Corporation, 1980.

[40] J. Crank and P. Nicolson, "A practical method for numerical evaluation of solutions of partial differential equations of the heat conduction type,” Proc. Camb. Phil. Soc., vol. 43, 
no. 1, pp. 50-67, 1947.

[41] B. R. Baliga and S. V Patankar, "A new finite-element formulation for convection- diffusion problems,” Numer. Heat Transf., vol. 3, pp. 393-409, 1980.

[42] S. Wen, "Optimization of electrodeposition process for tin coatings," McGill University, 2005.

[43] "Comission on Isotopic Abudances and Atomic Weights." [Online]. Available: http://www.ciaaw.org/atomic-weights.htm\#g. [Accessed: 12-May-2016].

[44] "National Institute of Standards and Technology." [Online]. Available: http://physics.nist.gov/cgi-bin/cuu/Value?na. [Accessed: 12-May-2016].

[45] M. Arita, H. Nakajima, M. Koiwa, and S. Miura, "Self diffusion of $\mathrm{Cu}$ and $\mathrm{Sn}$ in single crystal Cu3Sn ordered alloys,” Mater. Trans., vol. 32, no. 1, pp. 32-36, 1991.

[46] J. S. Dohie, "Rapid diffusion of zinc in iron,” 2005.

[47] M. J. Rizvi, C. Bailey, Y. C. Chan, and H. Lu, "Effect of adding 0.3 wt\% Ni into the Sn-0.7 wt\%Cu solder. Part I: Wetting behavior on $\mathrm{Cu}$ and Ni substrates,” J. Alloys Compd., vol. 438, no. 1-2, pp. 116-121, 2007.

[48] H. Ezawa, K. Higuchi, M. Seto, M. Uchida, and T. Togasaki, "Dilute Cu Alloying for SnCu Bumping by Annealing Electroplated Cu/Sn Stacks on Ti/Ni/Pd UBM,” Electron. Syst. Technol. Conf., pp. 550-555, 2006. 